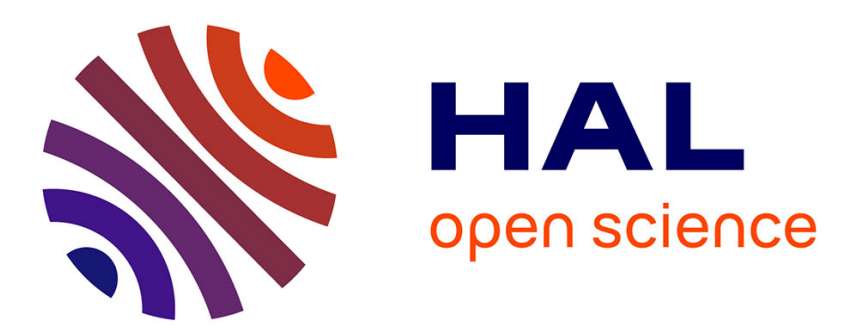

\title{
Existence of strong solutions to a fluid-structure system with a structure given by a finite number of parameters Guillaume Delay
}

\section{To cite this version:}

Guillaume Delay. Existence of strong solutions to a fluid-structure system with a structure given by a finite number of parameters. ESAIM: Mathematical Modelling and Numerical Analysis, 2020, 54

(1), 10.1051/m2an/2019059 . hal-01859849v4

\section{HAL Id: hal-01859849 \\ https://hal.science/hal-01859849v4}

Submitted on 6 Mar 2020

HAL is a multi-disciplinary open access archive for the deposit and dissemination of scientific research documents, whether they are published or not. The documents may come from teaching and research institutions in France or abroad, or from public or private research centers.
L'archive ouverte pluridisciplinaire HAL, est destinée au dépôt et à la diffusion de documents scientifiques de niveau recherche, publiés ou non, émanant des établissements d'enseignement et de recherche français ou étrangers, des laboratoires publics ou privés. 


\title{
Existence of strong solutions to a fluid-structure system with a structure given by a finite number of parameters*
}

\author{
Guillaume Delay ${ }^{\dagger}$
}

August 30, 2019

\begin{abstract}
We study the existence of strong solutions to a $2 \mathrm{~d}$ fluid-structure system. The fluid is modelled by the incompressible Navier-Stokes equations. The structure represents a steering gear and is described by two parameters corresponding to angles of deformation. Its equations are derived from a virtual work principle. The global domain represents a wind tunnel and imposes mixed boundary conditions to the fluid velocity. Our method reposes on the analysis of the linearized system. Under a compatibility condition on the initial data, we can guarantee local existence in time of strong solutions to the fluid-structure problem.
\end{abstract}

MSC numbers: 74F10, 74H20, 74H25, 74H30, 76D03.

\section{Introduction}

The goal of this study is to prove the existence of a unique solution to the fluid-structure problem presented in this section. We first expose the modelling of the structure. The goal is to approach the behaviour of a steering gear depending on two angles of rotation $\theta_{1}$ and $\theta_{2}$. We then give the equations of the whole fluid-structure interaction problem. In the sequel, we have to account for a time dependent domain for the fluid and for potential singularities. We present an adapted functional framework to tackle those difficulties. We state the main result of this study about existence and uniqueness of solutions to the fluid-structure interaction problem. Finally, we expose the scientific context of the study and the plan of the proof that is developped in the next sections.

\subsection{Modelling of the structure}

The considered structure lies inside an open bounded domain $\Omega \subset \mathbb{R}^{2}$ and deforms itself over time. The deformation depends on two angles $\theta_{1}$ and $\theta_{2}$ and approximates the behaviour of a steering gear structure. The couple of parameters $\left(\theta_{1}, \theta_{2}\right)$ lies in an admissible domain $\mathbb{D}_{\Theta}$ which is an open connected subset of $\mathbb{R}^{2}$. Let $S_{\text {ref }}$, a smooth closed connected subset of $\Omega$, be the reference configuration for the structure (for instance $S_{\text {ref }}$ is the volume occupied by the structure for $\theta_{1}=\theta_{2}=0$ ). We consider a function $\mathbf{X}$ defined on $\mathbb{D}_{\Theta} \times S_{\text {ref }}$ such that $\mathbf{X}\left(\theta_{1}, \theta_{2}, \mathbf{y}\right)$ is the position of the matter associated to the point $\mathbf{y}$ in the reference configuration $S_{\text {ref }}$.

The volume occupied by the structure for the parameters $\left(\theta_{1}, \theta_{2}\right) \in \mathbb{D}_{\Theta}$ is a closed bounded connected subset of $\Omega$ denoted $S\left(\theta_{1}, \theta_{2}\right)=\mathbf{X}\left(\theta_{1}, \theta_{2}, S_{\text {ref }}\right)$. We further assume that for every $\left(\theta_{1}, \theta_{2}\right) \in \mathbb{D}_{\Theta}, S\left(\theta_{1}, \theta_{2}\right) \subset \Omega$, i.e. there is no contact between the structure $S\left(\theta_{1}, \theta_{2}\right)$ and the boundary of the domain $\partial \Omega$.

We give on page 3 the modelling assumptions that have to be fulfilled. They enable an extension of the present work to a wider class of structures. Moreover, even if we consider only two parameters, all the results remain valid for any finite number of parameters. The extension of all proofs is indeed straightforward.

\subsubsection{Motivations}

Structures depending only on a finite number of parameters arise in the field of aeronautics. For instance, let us consider a steering gear structure. In a first approach, we can model this structure by two rigid solids. Solid $S_{1}$ is tied to the fixed frame by a pivoting link $O$ and solid $S_{2}$ is tied to solid $S_{1}$ by a pivoting link $P$. The whole model is represented in Fig. 1a. Note that $S_{1}$ can be thought of as the aerofoil of a wing and $S_{2}$ as a steering gear such as an aileron. For a given $S_{\text {ref }} \subset \Omega$, the function $\mathbf{X}^{a}$ representing the motion of this structure with respect to $\left(\theta_{1}, \theta_{2}\right)$ is given below

$$
\mathbf{X}^{a}\left(\theta_{1}, \theta_{2}, \mathbf{y}\right)=\chi_{S_{1}}(\mathbf{y}) R_{\theta_{1}} \mathbf{y}+\chi_{S_{2}}(\mathbf{y})\left(R_{\theta_{1}} \mathbf{y}_{P}^{\mathrm{ref}}+R_{\theta_{1}+\theta_{2}}\left(\mathbf{y}-\mathbf{y}_{P}^{\mathrm{ref}}\right)\right), \quad \forall \mathbf{y} \in S_{\mathrm{ref}}, \quad \forall\left(\theta_{1}, \theta_{2}\right) \in \mathbb{D}_{\Theta},
$$

\footnotetext{
${ }^{*}$ The author is partially supported by IFSMACS ANR-15-CE40-0010

$\dagger$ Institut de Mathématiques de Toulouse, Université Paul Sabatier - 118, route de Narbonne F-31062 Toulouse Cedex 9, France (guillaume.delay@math.univ-toulouse.fr or guillaume.delay@enpc.fr)
} 


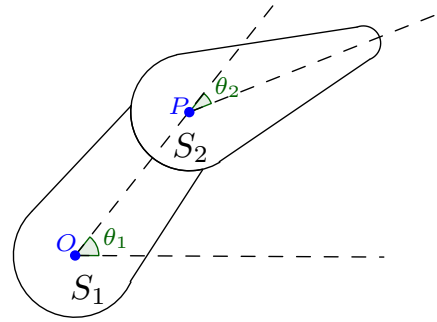

(a) A pivoting link $P$.

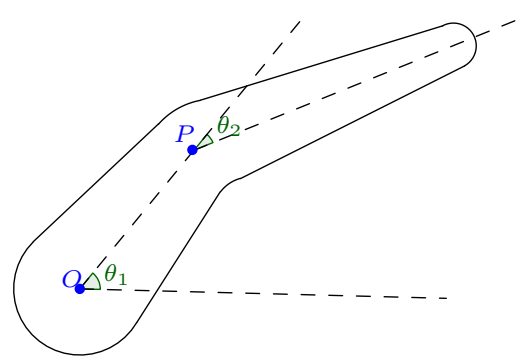

(b) A structure continuously deforming.

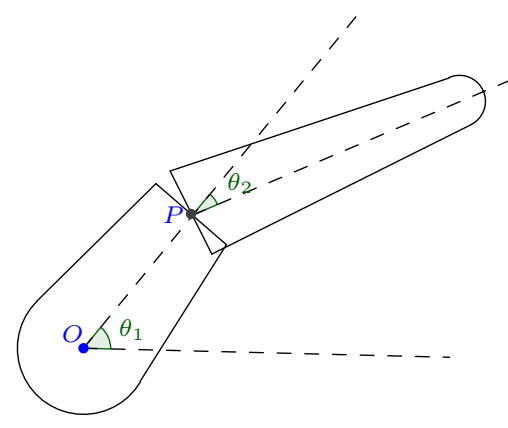

(c) A limit configuration.

Figure 1: Three different kinds of structure deformation.

where $R_{\theta}=\left(\begin{array}{cc}\cos \theta & -\sin \theta \\ \sin \theta & \cos \theta\end{array}\right)$ is the rotation matrix of angle $\theta, \mathbf{y}_{P}^{\text {ref }}=\left(y_{P, 1}, y_{P, 2}\right)$ is the coordinate of the point $P$ in the reference configuration $S_{\text {ref }}$ and $\chi_{E}$, the characteristic function over a set $E \subset \mathbb{R}^{2}$, is given below

$$
\forall \mathbf{y} \in \Omega, \quad \chi_{E}(\mathbf{y})= \begin{cases}1 & \text { if } \mathbf{y} \in E \\ 0 & \text { else. }\end{cases}
$$

In the previous example, the admissible domain $\mathbb{D}_{\Theta}$ for $\left(\theta_{1}, \theta_{2}\right)$ is chosen such that no overlaps of the structure occur.

Note that for $\theta_{2} \neq 0$, the function $\mathbf{X}^{a}\left(\theta_{1}, \theta_{2},.\right)$ is not a diffeomorphism as it is discontinuous through the interface $\partial S_{1} \cap \partial S_{2}$ between the two solids. In the same way, for $\dot{\theta}_{2} \neq 0$, the velocity field is discontinuous inside the structure (we denote $\dot{\theta}_{2}$ the time derivative of $\theta_{2}$ ). In other words, if we keep $S_{1}$ at rest and rotate $S_{2}$ around $P$, a discontinuity of the velocity appears through the interface between the two solids. This discontinuity can reduce the regularity expected for the fluid velocity. Indeed, if we assume no-slip boundary conditions between the fluid and the structure and if at time $t$ the trace of the velocity is discontinuous on $\partial S\left(\theta_{1}(t), \theta_{2}(t)\right)$, then a Sobolev embedding argument shows that we cannot hope for a better regularity in space for the velocity of the fluid than the Sobolev space $L^{2}\left(0, T ; \mathbf{H}^{1}\left(\Omega \backslash S\left(\theta_{1}(t), \theta_{2}(t)\right)\right)\right)^{1}$, while for strong solutions we usually expect the velocity in the Sobolev space $L^{2}\left(0, T ; \mathbf{H}^{2}\left(\Omega \backslash S\left(\theta_{1}(t), \theta_{2}(t)\right)\right)\right)^{1}$. This loss of regularity would harm the estimates of the nonlinear terms (see Appendix B). That is why we consider a smooth approximation $\mathbf{X}^{b}$ of the deformation $\mathbf{X}^{a}$.

In the sequel, $\mathbf{y}=\left(y_{1}, y_{2}\right)$ is the Lagrangian coordinate and $\mathbf{y}^{\perp}=\left(-y_{2}, y_{1}\right)$ is normal to $\mathbf{y}$. The behaviour of the smooth structure is represented in Fig. 1b, we give $\mathbf{X}^{b}$ below

$$
\mathbf{X}^{b}\left(\theta_{1}, \theta_{2}, \mathbf{y}\right)=g_{\theta_{1}}\left(y_{1}\right) \mathbf{e}_{r 1}+g_{\theta_{2}}\left(y_{1}\right) \mathbf{e}_{r 2}+y_{2} \frac{\mathbf{N}\left(y_{1}\right)}{\left\|\mathbf{N}\left(y_{1}\right)\right\|}, \quad \mathbf{y}=\left(y_{1}, y_{2}\right) \in S_{\text {ref }}, \quad\left(\theta_{1}, \theta_{2}\right) \in \mathbb{D}_{\Theta},
$$

where $g_{\theta_{1}}$ and $g_{\theta_{2}}$ are real-valued functions. The domain $\mathbb{D}_{\Theta}$ is chosen small enough, for instance $\mathbb{D}_{\Theta}=$ $B((0,0), \tilde{\varepsilon})$ for some $\tilde{\varepsilon}>0$, and $S_{\text {ref }}$ can be chosen as $S_{\text {ref }}=S(0,0)$. We use the notations: $\mathbf{e}_{r 1}=\left(\cos \theta_{1}, \sin \theta_{1}\right)$, $\mathbf{e}_{r 2}=\left(\cos \left(\theta_{1}+\theta_{2}\right), \sin \left(\theta_{1}+\theta_{2}\right)\right), \mathbf{N}\left(y_{1}\right)=g_{\theta_{1}}^{\prime}\left(y_{1}\right) \mathbf{e}_{\theta 1}+g_{\theta_{2}}^{\prime}\left(y_{1}\right) \mathbf{e}_{\theta 2}$, where $\mathbf{e}_{\theta 1}=\mathbf{e}_{r 1}^{\perp}$ and $\mathbf{e}_{\theta 2}=\mathbf{e}_{r 2}^{\perp}$. Moreover, we have $\left\|\mathbf{N}\left(y_{1}\right)\right\|=\left(\left(N_{1}\left(y_{1}\right)\right)^{2}+\left(N_{2}\left(y_{1}\right)\right)^{2}\right)^{1 / 2}$, where $N_{i}$ is the $i^{\text {th }}$ coordinate of $\mathbf{N}$.

The function $y_{1} \mapsto g_{\theta_{1}}\left(y_{1}\right) \mathbf{e}_{r 1}+g_{\theta_{2}}\left(y_{1}\right) \mathbf{e}_{r 2}$ gives for $\left(\theta_{1}, \theta_{2}\right) \in \mathbb{D}_{\Theta}$ the position of a curve. Every fibre of matter that is normal to this curve in the reference configuration stays normal when $\left(\theta_{1}, \theta_{2}\right)$ changes. This means that the deformation of this curve gives the deformation of the whole structure. The normal direction to the curve at abscissa $y_{1}$ is given by $\mathbf{N}\left(y_{1}\right)$. We assume that $\theta_{2}$ is small enough and that $g_{\theta_{1}}^{\prime}+g_{\theta_{2}}^{\prime} \geq \alpha$ for some $\alpha>0$. Since $\left\|\mathbf{N}\left(y_{1}\right)\right\|^{2}=\left(g_{\theta_{1}}^{\prime}+g_{\theta_{2}}^{\prime}\right)^{2}-2 g_{\theta_{1}}^{\prime} g_{\theta_{2}}^{\prime}\left(1-\cos \left(\theta_{2}\right)\right)$, this implies $\left\|\mathbf{N}\left(y_{1}\right)\right\| \geq \alpha^{\prime}$ for some $\alpha^{\prime}>0$. This model is inspired by the fish-like model described in [29, Section 7].

To enforce smoothness of $\mathbf{X}^{b}, g_{\theta_{1}}$ and $g_{\theta_{2}}$ are taken as $\mathscr{C}^{\infty}$ functions which are smooth approximations of respectively $y_{P, 1}+\left(y_{1}-y_{P, 1}\right) \chi_{\left[0, y_{P, 1}\right]}\left(y_{1}\right)$ and $\left(y_{1}-y_{P, 1}\right) \chi_{\left[y_{P, 1}, y_{\max }\right]}\left(y_{1}\right)$, where $\chi_{I}$ is defined in a similar way as (1.1) for $I \subset \mathbb{R}$. For instance, let $\varepsilon>0$ and consider $\mu_{\varepsilon}$ a $\mathscr{C}^{\infty}$ cut-off function such that

$$
\begin{cases}\mu_{\varepsilon}\left(y_{1}\right)=1, & \text { for } y_{1}<y_{P, 1} \\ \mu_{\varepsilon}\left(y_{1}\right) \in[0,1], & \text { for } y_{P, 1} \leq y_{1} \leq y_{P, 1}+\varepsilon \\ \mu_{\varepsilon}\left(y_{1}\right)=0, & \text { for } y_{P, 1}+\varepsilon<y_{1}\end{cases}
$$

Then, we can use

$$
\left\{\begin{array}{l}
g_{\theta_{1}}\left(y_{1}\right)=y_{P, 1}+\mu_{\varepsilon}\left(y_{1}\right)\left(y_{1}-y_{P, 1}\right), \\
g_{\theta_{2}}\left(y_{1}\right)=\left(1-\mu_{\varepsilon}\left(y_{1}\right)\right)\left(y_{1}-y_{P, 1}\right)
\end{array}\right.
$$

\footnotetext{
${ }^{1}$ These spaces are given here in an informal manner. They will be defined more precisely later.
} 
in (1.2) to get a smooth deformation as represented in Fig. 1b. The velocity field of the structure is not any more discontinuous, we can thus expect the fluid velocity to have the usual regularity of strong solutions. Moreover, this choice fulfils $g_{\theta_{1}}^{\prime}+g_{\theta_{2}}^{\prime}=1>0$.

Remark 1.1. When $\varepsilon$ tends to 0 , these functions become

$$
\left\{\begin{array}{l}
g_{\theta_{1}}\left(y_{1}\right)=\chi_{[a, b[}\left(y_{1}\right) y_{1}+\chi_{[b, c]}\left(y_{1}\right) y_{P, 1}, \\
g_{\theta_{2}}\left(y_{1}\right)=\chi_{[b, c]}\left(y_{1}\right)\left(y_{1}-y_{P, 2}\right),
\end{array}\right.
$$

for some real numbers $a, b, c$. In this case, we recover the behaviour of a pivoting structure with two rigid solids (see Fig. 1c), corresponding to a transformation denoted $\mathbf{X}^{c}$. However, with this definition, the two solids overlap each other, so that we will not use it neither in the sequel. Also let us remark that the limit $\mathbf{X}^{c}$ of our smooth approximation $\mathbf{X}^{b}$ is not the original model $\mathbf{X}^{a}$.

We shall keep in mind only the example of $\mathbf{X}^{b}$ (see Fig. 1b), though our original motivation was to deal with $\mathbf{X}^{a}$ (see Fig. 1a). More generally, our approach will be applicable to many more choices of deformations $\mathbf{X}$. Let us list below the assumptions used in the sequel.

\section{Modelling Assumptions.}

- For every $\mathbf{y} \in S_{\text {ref }}=S(0,0), \mathbf{X}(0,0, \mathbf{y})=\mathbf{y}$.

- $S_{\text {ref }}$ is a smooth simply connected closed subset of $\Omega$.

- For every $\left(\theta_{1}, \theta_{2}\right) \in \mathbb{D}_{\Theta}$, we have $\mathbf{X}\left(\theta_{1}, \theta_{2}, S_{\text {ref }}\right) \subset \Omega$ and $\inf _{\left(\theta_{1}, \theta_{2}\right) \in \mathbb{D}_{\Theta}} \mathrm{d}\left(\mathbf{X}\left(\theta_{1}, \theta_{2}, S_{\text {ref }}\right), \partial \Omega\right)>0$.

- For every $\left(\theta_{1}, \theta_{2}\right) \in \mathbb{D}_{\Theta}, \mathbf{X}\left(\theta_{1}, \theta_{2},.\right)$ is a $\mathscr{C}^{\infty}$ diffeomorphism from $S_{\text {ref }}$ to its image $S\left(\theta_{1}, \theta_{2}\right)$.

- The function $\mathbf{X}$ is $\mathscr{C}^{\infty}$ on $\mathrm{D}_{\Theta} \times S_{\text {ref }}$.

- The functions $\partial_{\theta_{1}} \mathbf{X}\left(\theta_{1}, \theta_{2},.\right)$ and $\partial_{\theta_{2}} \mathbf{X}\left(\theta_{1}, \theta_{2},.\right)$ form

$$
\text { a free family in } \mathbf{L}^{2}\left(\partial S_{\text {ref }}\right) \text { for every }\left(\theta_{1}, \theta_{2}\right) \text { in } \mathbb{D}_{\Theta} \text {. }
$$

In (1.4), we have assumed that $S_{\text {ref }}=S(0,0)$ to ease the study. In (1.6), we assume that the structure stays away from the boundary of the wind tunnel. Assumption (1.7) enables us to use a change of variables. This is a crucial step in our approach, as we shall see in Section 3.1. Assumption (1.8) ensures continuity of the velocity field inside the structure and on its boundary. This assumption could be weakened, as $\mathscr{C}^{n}$ would be sufficient for $n$ large enough, but we keep $\mathscr{C}^{\infty}$ for simplicity. In our approach, Assumption (1.9) is natural and mandatory to determine the equations of the structure, as we shall see below in Section 1.1.2. We now state the fact that the proposed deformation $\mathbf{X}^{b}$ fulfils those assumptions.

Lemma 1.2. For $\mathbb{D}_{\Theta}$ small enough, the function $\mathbf{X}^{b}$ defined in (1.2) satisfies the modelling assumptions (1.4)$(1.9)$.

A proof of this statement is provided in Appendix A. In the sequel, we do not only consider $\mathbf{X}^{b}$ but any $\mathbf{X}$ that satisfies (1.4)-(1.9). have

The inverse diffeomorphism of $\mathbf{X}\left(\theta_{1}, \theta_{2},.\right)$ whose existence is guaranteed by $(1.7)$ is denoted $\mathbf{Y}\left(\theta_{1}, \theta_{2},.\right)$, we

$$
\forall\left(\theta_{1}, \theta_{2}\right) \in \mathbb{D}_{\Theta}, \quad \forall \mathbf{y} \in S_{\text {ref }}, \quad \mathbf{Y}\left(\theta_{1}, \theta_{2}, \mathbf{X}\left(\theta_{1}, \theta_{2}, \mathbf{y}\right)\right)=\mathbf{y} .
$$

The diffeomorphisms $\mathbf{X}\left(\theta_{1}, \theta_{2},.\right)$ and $\mathbf{Y}\left(\theta_{1}, \theta_{2},.\right)$ are illustrated in Fig. 2.

Remark 1.3. The choice of $\mathbf{X}^{b}$ allows changes of volume for the structure and then for the fluid. In our setting where mixed boundary conditions are considered, this is not a problem. However, in the case, for instance, of homogeneous Dirichlet boundary conditions on $\partial \Omega$, the diffeomorphism $\mathbf{X}^{b}$ would have to be modified to ensure a constant volume to the structure.

\subsubsection{Dynamics of the structure}

In order to simplify the equations of the structure, we consider the following assumption for the dynamics of the structure.

\section{Modelling Assumption.}

- No friction and no elastic forces are considered in the structure.

The equations satisfied by the structure are obtained by a virtual work principle [3, p. 14-17]. We know that the admissible parameters of the structure are $\left(\theta_{1}, \theta_{2}\right) \in \mathbb{D}_{\Theta}$, and that the admissible velocities $\mathbf{v}_{s}$ satisfy

$$
\mathbf{v}_{s} \in \operatorname{Vect}\left(\partial_{\theta_{1}} \mathbf{X}\left(\theta_{1}, \theta_{2}, .\right), \partial_{\theta_{2}} \mathbf{X}\left(\theta_{1}, \theta_{2}, .\right)\right) \text {. }
$$




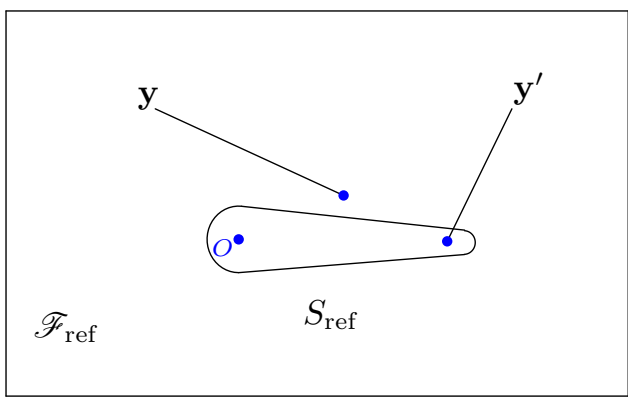

$\Omega$
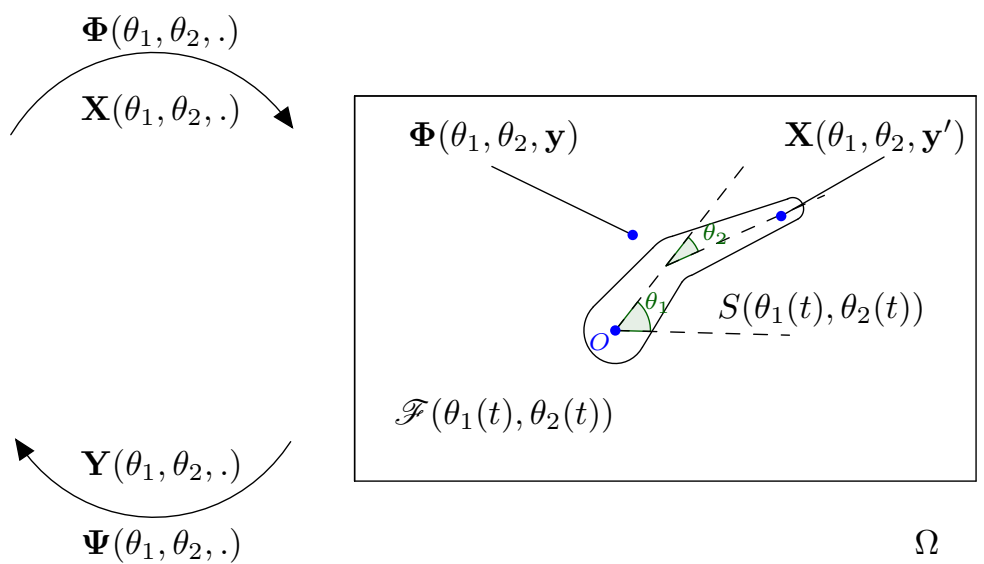

$\Omega$

Figure 2: Correspondance between real and reference structure configurations.

Thus, the virtual work principle can be formulated for every time $t \in[0, T]$ as

$$
\left\{\begin{array}{c}
\text { Find }\left(\theta_{1}(t), \theta_{2}(t)\right) \in \mathbb{D}_{\Theta}, \text { such that for every } \mathbf{w} \in \operatorname{Vect}\left(\partial_{\theta_{1}} \mathbf{X}\left(\theta_{1}(t), \theta_{2}(t), .\right), \partial_{\theta_{2}} \mathbf{X}\left(\theta_{1}(t), \theta_{2}(t), .\right)\right), \\
\int_{S_{\mathrm{ref}}} \rho\left(\frac{\mathrm{d}^{2}}{\mathrm{~d} t^{2}}\left(\mathbf{X}\left(\theta_{1}(t), \theta_{2}(t), \mathbf{y}\right)\right)-\mathbf{f}_{b o d y}\left(t, \mathbf{X}\left(\theta_{1}(t), \theta_{2}(t), \mathbf{y}\right)\right)\right) \cdot \mathbf{w}(\mathbf{y}) \mathrm{d} \mathbf{y} \\
-\int_{\partial S\left(\theta_{1}(t), \theta_{2}(t)\right)} \mathbf{f}_{\mathcal{F} \rightarrow S}\left(\gamma_{x}\right) \cdot \mathbf{w}\left(\mathbf{Y}\left(\theta_{1}(t), \theta_{2}(t), \gamma_{x}\right)\right) \mathrm{d} \gamma_{x}=0
\end{array}\right.
$$

where $\mathbf{f}_{b o d y}$ is a distributed source term in the body (modelling for instance the gravity), $\rho$ is a positive constant that represents the mass per unit volume of the structure in the reference configuration $S_{\text {ref }}$ and $\mathbf{f}_{\mathcal{F} \rightarrow S}$ is the force exerted by the fluid on the structure along $\partial S\left(\theta_{1}(t), \theta_{2}(t)\right)$.

Note that the presence of $\mathbf{f}_{\text {body }}$ is compatible with Assumption (1.11), as this term represents external forces. It does not depend on $\theta_{1}, \theta_{2}$ and their derivatives.

Remark 1.4. Assumption (1.11) has been used in (1.12) as no interior works have been considered.

Let us denote respectively $\dot{\theta}_{j}$ and $\ddot{\theta}_{j}$ the first and second time derivatives of the function $\theta_{j}$. Then, the velocity of the structure can be written as

$$
\mathbf{v}_{s}(t, \mathbf{y})=\frac{\mathrm{d}}{\mathrm{d} t} \mathbf{X}\left(\theta_{1}(t), \theta_{2}(t), \mathbf{y}\right)=\sum_{j=1}^{2} \dot{\theta}_{j}(t) \partial_{\theta_{j}} \mathbf{X}\left(\theta_{1}(t), \theta_{2}(t), \mathbf{y}\right), \quad \forall t \in[0, T], \quad \forall \mathbf{y} \in S_{\text {ref }},
$$

and its acceleration as

$$
\frac{\mathrm{d}}{\mathrm{d} t} \mathbf{v}_{s}(t, \mathbf{y})=\frac{\mathrm{d}^{2}}{\mathrm{~d} t^{2}}\left(\mathbf{X}\left(\theta_{1}(t), \theta_{2}(t), \mathbf{y}\right)\right)=\sum_{j=1}^{2} \ddot{\theta}_{j}(t) \partial_{\theta_{j}} \mathbf{X}\left(\theta_{1}(t), \theta_{2}(t), \mathbf{y}\right)+\sum_{j, k=1}^{2} \dot{\theta}_{j}(t) \dot{\theta}_{k}(t) \partial_{\theta_{j} \theta_{k}} \mathbf{X}\left(\theta_{1}(t), \theta_{2}(t), \mathbf{y}\right)
$$

Now, problem (1.12) can be rewritten as follows

$$
\begin{aligned}
& \text { Find }\left(\theta_{1}, \theta_{2}\right) \in \mathbb{D}_{\Theta}, \text { such that for every } i \in\{1,2\}, \text { we have, } \\
& \qquad \begin{aligned}
\int_{S_{\text {ref }}} \rho \sum_{j=1}^{2} \ddot{\theta}_{j} \partial_{\theta_{j}} \mathbf{X}\left(\theta_{1}, \theta_{2}, \mathbf{y}\right) \cdot \partial_{\theta_{i}} \mathbf{X}\left(\theta_{1}, \theta_{2}, \mathbf{y}\right) \mathrm{d} \mathbf{y}= & -\int_{S_{\mathrm{ref}}} \rho \sum_{j, k=1}^{2} \dot{\theta}_{j} \dot{\theta}_{k} \partial_{\theta_{j} \theta_{k}} \mathbf{X}\left(\theta_{1}, \theta_{2}, \mathbf{y}\right) \cdot \partial_{\theta_{i}} \mathbf{X}\left(\theta_{1}, \theta_{2}, \mathbf{y}\right) \mathrm{d} \mathbf{y} \\
& +\int_{S_{\mathrm{ref}}}^{\mathbf{f}_{b o d y}}\left(t, \mathbf{X}\left(\theta_{1}, \theta_{2}, \mathbf{y}\right)\right) \cdot \partial_{\theta_{i}} \mathbf{X}\left(\theta_{1}, \theta_{2}, \mathbf{y}\right) \mathrm{d} \mathbf{y} \\
& +\int_{\partial S\left(\theta_{1}, \theta_{2}\right)}^{\mathbf{f}_{\mathcal{F} \rightarrow S}\left(\gamma_{x}\right) \cdot \partial_{\theta_{i}} \mathbf{X}\left(\theta_{1}, \theta_{2}, \mathbf{Y}\left(\theta_{1}, \theta_{2}, \gamma_{x}\right)\right) \mathrm{d} \gamma_{x}}
\end{aligned}
\end{aligned}
$$

Let us denote the structure body source term

$$
\left(f_{s}\right)_{i}=\int_{S_{\mathrm{ref}}} \mathbf{f}_{b o d y}\left(t, \mathbf{X}\left(\theta_{1}, \theta_{2}, \mathbf{y}\right)\right) \cdot \partial_{\theta_{i}} \mathbf{X}\left(\theta_{1}, \theta_{2}, \mathbf{y}\right) \mathrm{d} \mathbf{y}
$$

On a matrix form, the equations of the structure read 


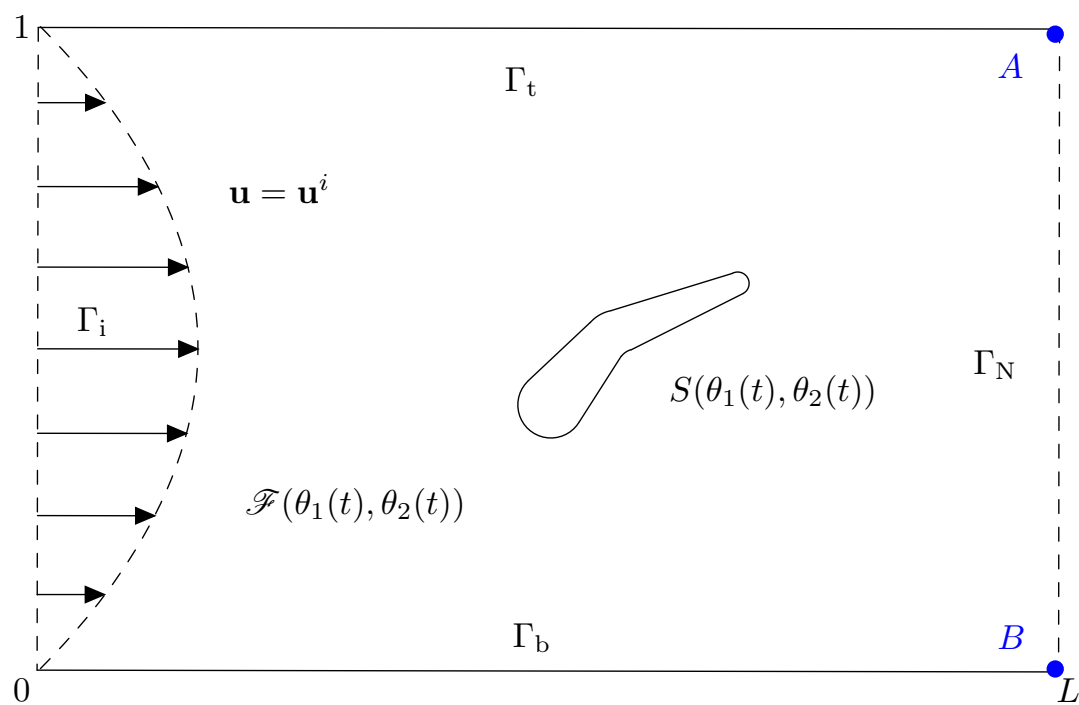

Figure 3: The geometrical configuration.

$$
\mathcal{M}_{\theta_{1}, \theta_{2}}\left(\begin{array}{c}
\ddot{\theta}_{1} \\
\ddot{\theta}_{2}
\end{array}\right)=\mathbf{M}_{\mathbf{I}}\left(\theta_{1}, \theta_{2}, \dot{\theta}_{1}, \dot{\theta}_{2}\right)+\mathbf{M}_{\mathbf{A}}\left(\theta_{1}, \theta_{2}, \mathbf{f}_{\mathcal{F} \rightarrow S}\right)+\mathbf{f}_{\mathbf{s}} \quad \text { on }(0, T),
$$

where $\mathbf{f}_{\mathbf{s}}=\left(\left(f_{s}\right)_{1},\left(f_{s}\right)_{2}\right)$ and

$$
\mathcal{M}_{\theta_{1}, \theta_{2}}=\left(\begin{array}{cc}
\left(\partial_{\theta_{1}} \mathbf{X}\left(\theta_{1}, \theta_{2}, .\right), \partial_{\theta_{1}} \mathbf{X}\left(\theta_{1}, \theta_{2}, .\right)\right)_{S} & \left(\partial_{\theta_{2}} \mathbf{X}\left(\theta_{1}, \theta_{2}, .\right), \partial_{\theta_{1}} \mathbf{X}\left(\theta_{1}, \theta_{2}, .\right)\right)_{S} \\
\left(\partial_{\theta_{1}} \mathbf{X}\left(\theta_{1}, \theta_{2}, .\right), \partial_{\theta_{2}} \mathbf{X}\left(\theta_{1}, \theta_{2}, .\right)\right)_{S} & \left(\partial_{\theta_{2}} \mathbf{X}\left(\theta_{1}, \theta_{2}, .\right), \partial_{\theta_{2}} \mathbf{X}\left(\theta_{1}, \theta_{2}, .\right)\right)_{S}
\end{array}\right) \in \mathbb{R}^{2 \times 2}
$$

$\mathbf{M}_{\mathbf{I}}\left(\theta_{1}, \theta_{2}, \dot{\theta}_{1}, \dot{\theta}_{2}\right)=\left(\begin{array}{c}-\left(\dot{\theta}_{1}^{2} \partial_{\theta_{1} \theta_{1}} \mathbf{X}\left(\theta_{1}, \theta_{2}, .\right)+2 \dot{\theta}_{1} \dot{\theta}_{2} \partial_{\theta_{1} \theta_{2}} \mathbf{X}\left(\theta_{1}, \theta_{2}, .\right)+\dot{\theta}_{2}^{2} \partial_{\theta_{2} \theta_{2}} \mathbf{X}\left(\theta_{1}, \theta_{2}, .\right), \partial_{\theta_{1}} \mathbf{X}\left(\theta_{1}, \theta_{2}, .\right)\right)_{S} \\ -\left(\dot{\theta}_{1}^{2} \partial_{\theta_{1} \theta_{1}} \mathbf{X}\left(\theta_{1}, \theta_{2}, .\right)+2 \dot{\theta}_{1} \dot{\theta}_{2} \partial_{\theta_{1} \theta_{2}} \mathbf{X}\left(\theta_{1}, \theta_{2}, .\right)+\dot{\theta}_{2}^{2} \partial_{\theta_{2} \theta_{2}} \mathbf{X}\left(\theta_{1}, \theta_{2}, .\right), \partial_{\theta_{2}} \mathbf{X}\left(\theta_{1}, \theta_{2}, .\right)\right)_{S}\end{array}\right) \in \mathbb{R}^{2}$,

where $(., .)_{S}$ is the scalar product

$$
(\boldsymbol{\Phi}, \boldsymbol{\Psi})_{S}=\int_{S_{\mathrm{ref}}} \rho \mathbf{\Phi}(\mathbf{y}) \cdot \mathbf{\Psi}(\mathbf{y}) \mathrm{d} \mathbf{y}
$$

and

$$
\mathbf{M}_{\mathbf{A}}\left(\theta_{1}, \theta_{2}, \mathbf{f}_{\mathcal{F} \rightarrow S}\right)=\left(\begin{array}{l}
\int_{\partial S\left(\theta_{1}, \theta_{2}\right)} \partial_{\theta_{1}} \mathbf{X}\left(\theta_{1}, \theta_{2}, \mathbf{Y}\left(\theta_{1}, \theta_{2}, \gamma_{x}\right)\right) \cdot \mathbf{f}_{\mathcal{F} \rightarrow S}\left(\gamma_{x}\right) \mathrm{d} \gamma_{x} \\
\int_{\partial S\left(\theta_{1}, \theta_{2}\right)} \partial_{\theta_{2}} \mathbf{X}\left(\theta_{1}, \theta_{2}, \mathbf{Y}\left(\theta_{1}, \theta_{2}, \gamma_{x}\right)\right) \cdot \mathbf{f}_{\mathcal{F} \rightarrow S}\left(\gamma_{x}\right) \mathrm{d} \gamma_{x}
\end{array}\right) \in \mathbb{R}^{2}
$$

The matrix $\mathcal{M}_{\theta_{1}, \theta_{2}}$ in (1.16) is the Gram matrix of the family $\left(\partial_{\theta_{1}} \mathbf{X}\left(\theta_{1}, \theta_{2}\right), \partial_{\theta_{2}} \mathbf{X}\left(\theta_{1}, \theta_{2}\right)\right)$ with respect to the scalar product $(., .)_{S}$. It is invertible due to Assumption (1.9) (if two $\mathscr{C}^{\infty}$ functions are collinear in $\mathbf{L}^{2}\left(S_{\text {ref }}\right)$ then they are collinear in $\left.\mathbf{L}^{2}\left(\partial S_{\text {ref }}\right)\right)$.

We also consider the following initial position and velocity for the structure

$$
\left\{\begin{array}{cc}
\theta_{1}(0)=\theta_{1,0}, & \theta_{2}(0)=\theta_{2,0} \\
\dot{\theta}_{1}(0)=\omega_{1,0}, & \dot{\theta}_{2}(0)=\omega_{2,0}
\end{array}\right.
$$

\subsection{Modelling of the full fluid-structure problem}

We here present the full system of equations that are studied in the sequel.

\subsubsection{Equations of the fluid}

In our study, the global domain $\Omega=(0, L) \times(0,1)$ represents a wind tunnel of length $L>0$, see Fig. 3 . Hence its boundary is composed of four regions: an inflow region $\Gamma_{\mathrm{i}}=\{0\} \times(0,1)$, a bottom region $\Gamma_{\mathrm{b}}=(0, L) \times\{0\}$, a top region $\Gamma_{\mathrm{t}}=(0, L) \times\{1\}$ and an outflow region $\Gamma_{\mathrm{N}}=\{L\} \times(0,1)$. We denote $\Gamma_{\mathrm{w}}=\Gamma_{\mathrm{t}} \cup \Gamma_{\mathrm{b}}$ the part of the boundary corresponding to walls and $\Gamma_{\mathrm{D}}=\Gamma_{\mathrm{i}} \cup \Gamma_{\mathrm{w}}$ the part of the boundary where Dirichlet conditions are imposed.

At time $t$, the structure occupies the volume $S\left(\theta_{1}(t), \theta_{2}(t)\right)$, therefore the fluid fills the domain $\mathscr{F}\left(\theta_{1}(t), \theta_{2}(t)\right)=$ $\Omega \backslash S\left(\theta_{1}(t), \theta_{2}(t)\right)$. 
The velocity of the fluid is modelled by the incompressible Navier-Stokes equations

$$
\begin{cases}\frac{\partial \mathbf{u}}{\partial t}(t, \mathbf{x})+(\mathbf{u} \cdot \nabla) \mathbf{u}(t, \mathbf{x})-\operatorname{div} \sigma_{F}(\mathbf{u}(t, \mathbf{x}), p(t, \mathbf{x}))=\mathbf{f}_{\mathscr{F}}(t, \mathbf{x}), & t \in(0, T), \quad \mathbf{x} \in \mathscr{F}\left(\theta_{1}(t), \theta_{2}(t)\right), \\ \operatorname{div} \mathbf{u}(t, \mathbf{x})=0, & t \in(0, T), \quad \mathbf{x} \in \mathscr{F}\left(\theta_{1}(t), \theta_{2}(t)\right), \\ \mathbf{u}(t, \mathbf{x})=\mathbf{u}^{i}(t, \mathbf{x}), & t \in(0, T), \quad \mathbf{x} \in \Gamma_{\mathrm{i}}, \\ \mathbf{u}(t, \mathbf{x})=0, & t \in(0, T), \quad \mathbf{x} \in \Gamma_{\mathrm{w}}, \\ \sigma_{F}(\mathbf{u}(t, \mathbf{x}), p(t, \mathbf{x})) \mathbf{n}(\mathbf{x})=0, & t \in(0, T), \quad \mathbf{x} \in \Gamma_{\mathrm{N}}, \\ \mathbf{u}(0, \mathbf{x})=\mathbf{u}_{0}(\mathbf{x}), & \end{cases}
$$

where $\mathbf{u}(t, \mathbf{x})$ and $p(t, \mathbf{x})$ are velocity and pressure of the fluid at point $\mathbf{x}$ and time $t$, and

$$
\sigma_{F}(\mathbf{u}, p)=\nu\left(\nabla \mathbf{u}+(\nabla \mathbf{u})^{T}\right)-p I
$$

is the stress tensor of the fluid, where $\nu>0$ is the kinematic viscosity of the fluid. The vector $\mathbf{n}$ denotes the unit outward normal to $\Omega$. The term $\mathbf{f}_{\mathscr{F}}(t, \mathbf{x})$ in $(1.21)_{1}$ is a force per unit mass exerted on the fluid. Moreover, a nonhomogeneous Dirichlet boundary condition with datum $\mathbf{u}^{i}$ is imposed on the inflow region $\Gamma_{\mathrm{i}}$ and we consider an initial datum $\mathbf{u}_{0}$ for the fluid velocity. Of course, these equations should be completed with suitable boundary conditions on $\partial S\left(\theta_{1}(t), \theta_{2}(t)\right)$ that are made precise in Section 1.2.2.

\subsubsection{Interface conditions between the fluid and the structure}

The velocity $\mathbf{u}$ of the fluid fulfils an adherence condition with the boundary of the structure whose velocity is given in (1.13),

$$
\mathbf{u}\left(t, \mathbf{X}\left(\theta_{1}(t), \theta_{2}(t), \mathbf{y}\right)\right)=\sum_{j=1}^{2} \dot{\theta}_{j}(t) \partial_{\theta_{j}} \mathbf{X}\left(\theta_{1}(t), \theta_{2}(t), \mathbf{y}\right), \quad t \in(0, T), \quad \mathbf{y} \in \partial S_{\mathrm{ref}} .
$$

Note that this no-slip boundary condition corresponds to the continuity of the velocity through the interface between the fluid and the structure and can also be rewritten as

$$
\mathbf{u}(t, \mathbf{x})=\sum_{j=1}^{2} \dot{\theta}_{j}(t) \partial_{\theta_{j}} \mathbf{X}\left(\theta_{1}(t), \theta_{2}(t), \mathbf{Y}\left(\theta_{1}(t), \theta_{2}(t), \mathbf{x}\right)\right), \quad t \in(0, T), \quad \mathbf{x} \in \partial S\left(\theta_{1}(t), \theta_{2}(t)\right)
$$

The forces exerted by the fluid on the structure are given by the stress tensor of the fluid

$$
\mathbf{f}_{\mathcal{F} \rightarrow S}(t, \mathbf{x})=-\sigma_{F}(\mathbf{u}, p) \mathbf{n}_{\theta_{1}, \theta_{2}}(t, \mathbf{x}), \quad t \in(0, T), \quad \mathbf{x} \in \partial S\left(\theta_{1}(t), \theta_{2}(t)\right),
$$

where $\mathbf{n}_{\theta_{1}, \theta_{2}}(\mathbf{x})$ is the outward unit normal to the fluid domain $\mathscr{F}\left(\theta_{1}(t), \theta_{2}(t)\right)$ on $\partial S\left(\theta_{1}(t), \theta_{2}(t)\right)$.

\subsubsection{The complete set of equations}

The full set of equations is given by $(1.15),(1.20),(1.21),(1.22)$ and (1.23). Note that the coupling between the fluid and the structure appears in equations (1.21) (the fluid domain depends on $\theta_{1}$ and $\left.\theta_{2}\right),(1.22)$ and (1.23).

The considered system is given by the following set of equations

$$
\begin{cases}\frac{\partial \mathbf{u}}{\partial t}(t, \mathbf{x})+(\mathbf{u}(t, \mathbf{x}) \cdot \nabla) \mathbf{u}(t, \mathbf{x})-\operatorname{div} \sigma_{F}(\mathbf{u}(t, \mathbf{x}), p(t, \mathbf{x}))=\mathbf{f}_{\mathscr{F}}(t, \mathbf{x}), & t \in(0, T), \quad \mathbf{x} \in \mathscr{F}\left(\theta_{1}(t), \theta_{2}(t)\right), \\ \operatorname{div} \mathbf{u}(t, \mathbf{x})=0, & t \in(0, T), \quad \mathbf{x} \in \mathscr{F}\left(\theta_{1}(t), \theta_{2}(t)\right) \\ \mathbf{u}(t, \mathbf{x})=\sum_{j=1}^{2} \dot{\theta}_{j}(t) \partial_{\theta_{j}} \mathbf{X}\left(\theta_{1}(t), \theta_{2}(t), \mathbf{Y}\left(\theta_{1}(t), \theta_{2}(t), \mathbf{x}\right)\right), & t \in(0, T), \quad \mathbf{x} \in \partial S\left(\theta_{1}(t), \theta_{2}(t)\right) \\ \mathbf{u}(t, \mathbf{x})=\mathbf{u}^{i}(t, \mathbf{x}), & t \in(0, T), \quad \mathbf{x} \in \Gamma_{\mathrm{i}}, \\ \mathbf{u}(t, \mathbf{x})=0, & t \in(0, T), \quad \mathbf{x} \in \Gamma_{\mathrm{w}}, \\ \sigma_{F}(\mathbf{u}(t, \mathbf{x}), p(t, \mathbf{x})) \mathbf{n}(\mathbf{x})=0, & t \in(0, T), \quad \mathbf{x} \in \Gamma_{\mathrm{N}}, \\ \mathbf{u}(0, \mathbf{x})=\mathbf{u}_{0}(\mathbf{x}), & \mathbf{x} \in \mathscr{F}_{\left(\theta_{1,0}, \theta_{2,0}\right)} \\ \mathcal{M}_{\theta_{1}, \theta_{2}}\left(\ddot{\theta}_{1}\right)=\mathbf{\theta}_{\mathbf{I}}\left(\theta_{1}, \theta_{2}, \dot{\theta}_{1}, \dot{\theta}_{2}\right)+\mathbf{M}_{\mathbf{A}}\left(\theta_{1}, \theta_{2},-\sigma_{F}(\mathbf{u}, p) \mathbf{n}_{\theta_{1}, \theta_{2}}\right)+\mathbf{f}_{\mathbf{s}}, & t \in(0, T), \\ \theta_{1}(0)=\theta_{1,0}, \quad \theta_{2}(0)=\theta_{2,0} & \\ \dot{\theta}_{1}(0)=\omega_{1,0}, \quad \dot{\theta}_{2}(0)=\omega_{2,0} & \end{cases}
$$




\subsection{Functional framework}

In this section, we present the functional framework of the study. In the sequel, $\mathscr{F} 0=\mathscr{F}\left(\theta_{1,0}, \theta_{2,0}\right)$ denotes the initial fluid domain and $S_{0}=S\left(\theta_{1,0}, \theta_{2,0}\right)$ the initial configuration of the structure. For the sake of simplicity, the initial parameters of the structure are taken equal to zero,

$$
\theta_{1,0}=\theta_{2,0}=0
$$

This can be done without loss of generality by the change of variables

$$
\left(\theta_{1}, \theta_{2}\right) \mapsto\left(\theta_{1}-\theta_{1,0}, \theta_{2}-\theta_{2,0}\right)
$$

Moreover, the reference configuration for the structure $S_{\text {ref }}$ and for the fluid $\mathscr{F}$ ref are taken as the initial configuration,

$$
S_{\text {ref }}=S_{0}=S(0,0), \mathscr{F}_{\text {ref }}=\mathscr{F}_{0}=\mathscr{F}(0,0) .
$$

Sobolev spaces. In the sequel, $H^{s}\left(\mathscr{F}_{0}\right)$ is the usual Sobolev space of order $s \geq 0$. We identify $L^{2}\left(\mathscr{F}_{0}\right)$ with $H^{0}\left(\mathscr{F}_{0}\right)$. We denote $\mathbf{L}^{2}\left(\mathscr{F}_{0}\right)=\left(L^{2}\left(\mathscr{F}_{0}\right)\right)^{2}, \mathbf{H}^{s}\left(\mathscr{F}_{0}\right)=\left(H^{s}\left(\mathscr{F}_{0}\right)\right)^{2}$ and so on.

Corners issues. The domain considered for the fluid has four corners of angle $\pi / 2$. The ones that are located between Dirichlet and Neumann boundary conditions induce singularities, we denote them $A=(L, 1)$ and $B=(L, 0)$ (see Fig. 3). We also denote $\mathscr{J}_{d, n}=\{A, B\}$ the set of these corners and we define the distance of a point $\mathbf{x}$ from them,

$$
\text { for } j \in \mathscr{J}_{d, n}, \quad \text { for } \mathbf{x} \in \Omega, \quad r_{j}(\mathbf{x})=\mathrm{d}(\mathbf{x}, j) \text {. }
$$

Note that corners between two Dirichlet boundary conditions do not induce singularities as soon as suitable compatibility conditions are satisfied. We report to [23] for more details.

Weighted Sobolev spaces. The strong solution to the Stokes problem in the domain with corners $A$ and $B$ and with a source term in $\mathbf{L}^{2}\left(\mathscr{F}_{0}\right)$ belongs to a classical Sobolev space of lower order than what we usually have with smooth domains. In order to get the usual gain of regularity between solutions and source terms, we have to study the solution in adapted Sobolev spaces. As the loss of regularity is located around corners $A$ and $B$, we can recover the usual regularity if we consider norms that are suitably weighted near these corners. The weighted Sobolev spaces are then defined for $\beta>0$ as

$$
\begin{array}{r}
\mathbf{H}_{\beta}^{2}\left(\mathscr{F}_{0}\right)=\left\{\mathbf{u} \text { with }\|\mathbf{u}\|_{\mathbf{H}_{\beta}^{2}\left(\mathscr{F}_{0}\right)}<+\infty\right\} \\
H_{\beta}^{1}\left(\mathscr{F}_{0}\right)=\left\{p \text { with }\|p\|_{H_{\beta}^{1}\left(\mathscr{F}_{0}\right)}<+\infty\right\},
\end{array}
$$

where the norms $\|\cdot\|_{\mathbf{H}_{\beta}^{2}\left(\mathscr{F}_{0}\right)}$ and $\|\cdot\|_{H_{\beta}^{1}\left(\mathscr{F}_{0}\right)}$ are given by

$$
\|\mathbf{u}\|_{\mathbf{H}_{\beta}^{2}\left(\mathscr{F}_{0}\right)}^{2}=\sum_{|\alpha|=0}^{2} \sum_{i=1}^{2} \int_{\mathscr{F}_{0}}\left(\prod_{j \in \mathscr{J}_{d, n}} r_{j}^{2 \beta}(\mathbf{y})\right)\left|\partial^{\alpha} u_{i}(\mathbf{y})\right|^{2} \mathrm{~d} \mathbf{y},
$$

and

$$
\|p\|_{H_{\beta}^{1}\left(\mathscr{F}_{0}\right)}^{2}=\sum_{|\alpha|=0}^{1} \int_{\mathscr{F}_{0}}\left(\prod_{j \in \mathscr{J}_{d, n}} r_{j}^{2 \beta}(\mathbf{y})\right)\left|\partial^{\alpha} p(\mathbf{y})\right|^{2} \mathrm{~d} \mathbf{y} .
$$

Here the sum is on every multi-index $\alpha$ of length $|\alpha| \leq 2$ for (1.26) and $|\alpha| \leq 1$ for (1.27) and $r_{j}$ is defined in (1.25). Moreover, as a consequence of [2, Proposition A.1], for every $\mathbf{u} \in \mathbf{H}_{\beta}^{2}\left(\mathscr{F}_{0}\right)$ fulfilling $\mathbf{u}=0$ on $\Gamma_{\mathrm{w}}$, we have $\mathbf{u} \in \mathbf{H}^{2-\beta}\left(\mathscr{F}_{0}\right)$ and

$$
\|\mathbf{u}\|_{\mathbf{H}^{2-\beta}\left(\mathscr{F}_{0}\right)} \leq C\|\mathbf{u}\|_{\mathbf{H}_{\beta}^{2}\left(\mathscr{F}_{0}\right)}
$$

where $C>0$ does not depend on $\mathbf{u}$. Note that the geometrical configuration considered in the present article is used in the proof of this inequality.

Steady Stokes problem with corners. The following lemma from [25] explains how and why the spaces $\mathbf{H}_{\beta}^{2}$ and $H_{\beta}^{1}$ appear in the context of corners. It gives the result expected for the steady Stokes problem in $\mathscr{F}_{0}$ with weigthed Sobolev spaces and the regularity obtained in the classical Sobolev spaces.

Lemma 1.5. [25, Theorem 2.5.] Let us assume that $\mathbf{f}_{\mathscr{F}} \in \mathbf{L}^{2}\left(\mathscr{F}_{0}\right)$. The unique solution $(\mathbf{u}, p)$ to the Stokes problem

$$
\begin{cases}-\operatorname{div} \sigma_{F}(\mathbf{u}, p)=\mathbf{f}_{\mathscr{F}} & \text { in } \mathscr{F}_{0} \\ \operatorname{div} \mathbf{u}=0 & \text { in } \mathscr{F}_{0}, \\ \mathbf{u}=0 & \text { on } \Gamma_{\mathrm{D}} \cup \partial S_{0} \\ \sigma_{F}(\mathbf{u}, p) \mathbf{n}=0 & \text { on } \Gamma_{\mathrm{N}}\end{cases}
$$


belongs to $\mathbf{H}_{\beta}^{2}\left(\mathscr{F}_{0}\right) \times H_{\beta}^{1}\left(\mathscr{F}_{0}\right)$ for some $\beta \in(0,1 / 2)$ and to $\mathbf{H}^{3 / 2+\varepsilon_{0}}\left(\mathscr{F}_{0}\right) \times H^{1 / 2+\varepsilon_{0}}\left(\mathscr{F}_{0}\right)$ for some $\varepsilon_{0} \in(0,1 / 2)$. Moreover, we have the following estimate

$$
\|\mathbf{u}\|_{\mathbf{H}_{\beta}^{2}\left(\mathscr{F}_{0}\right) \cap \mathbf{H}^{3 / 2+\varepsilon_{0}\left(\mathscr{F}_{0}\right)}}+\|p\|_{H_{\beta}^{1}\left(\mathscr{F}_{0}\right) \cap H^{1 / 2+\varepsilon_{0}\left(\mathscr{F}_{0}\right)}} \leq C\left\|\mathbf{f}_{\mathscr{F}}\right\|_{\mathbf{L}^{2}\left(\mathscr{F}_{0}\right)}
$$

Let us denote $\mathbf{n}_{0}$ the outward unit normal to $\mathscr{F}_{0}$ on $\partial S_{0}$. The regularity proven in Lemma 1.5 gives a meaning to all integrations by parts as $p_{\mid \partial \mathscr{F}_{0}}$ and $\partial_{\mathbf{n}_{0}} \mathbf{u}_{\mid \partial \mathscr{F}_{0}}$ are well defined traces for $(\mathbf{u}, p) \in \mathbf{H}^{3 / 2+\varepsilon_{0}}\left(\mathscr{F}_{0}\right) \times H^{1 / 2+\varepsilon_{0}}\left(\mathscr{F}_{0}\right)$.

Also note that according to [16, Theorem 1.4.3.1], there exists a continuous extension operator from $\mathbf{H}^{s}\left(\mathscr{F}_{0}\right)$ to $\mathbf{H}^{s}\left(\mathbb{R}^{2}\right)$ for every $s>0$. This implies that all the classical Sobolev embeddings and interpolations are valid despite the presence of corners as they can be led in $\mathbb{R}^{2}$.

Remark 1.6. Lemma 1.5 uses the geometry of the problem. Especially, we do not consider junctions between two segments where Neumann boundary conditions are imposed and all junctions between segments are right angles. To consider other angles, the reader can report to [23]. Note that the value of $\varepsilon_{0}$ and $\beta$ depends on those angles.

Time-dependent spaces for the study in the fixed domain $\mathscr{F}_{0}$. In the sequel, we study the problem in the fixed domain $\mathscr{F}_{0}$. Let $T>0$ be the final time of the system. The following spaces are considered

$$
\begin{aligned}
\mathbb{U}_{T} & =\left\{\mathbf{u} \in L^{2}\left(0, T ; \mathbf{H}_{\beta}^{2}\left(\mathscr{F}_{0}\right)\right) \cap \mathscr{C}^{0}\left([0, T] ; \mathbf{H}^{1}\left(\mathscr{F}_{0}\right)\right) \cap H^{1}\left(0, T ; \mathbf{L}^{2}\left(\mathscr{F}_{0}\right)\right) \mid \mathbf{u}=0 \text { on }[0, T] \times \Gamma_{\mathrm{w}}\right\}, \\
\mathbb{P}_{T} & =L^{2}\left(0, T ; H_{\beta}^{1}\left(\mathscr{F}_{0}\right)\right), \\
\Theta_{T} & =H^{2}\left(0, T ; \mathbb{R}^{2}\right) \\
\mathbb{F}_{T} & =L^{2}\left(0, T ; \mathbf{L}^{2}\left(\mathscr{F}_{0}\right)\right), \\
\mathbb{G}_{T} & =H^{1}\left(0, T ; \mathbf{H}^{3 / 2}\left(\partial S_{0}\right)\right), \\
\mathbb{S}_{T} & =L^{2}\left(0, T ; \mathbb{R}^{2}\right) .
\end{aligned}
$$

Note that we include the boundary condition $\mathbf{u}=0$ on $[0, T] \times \Gamma_{\mathrm{w}}$ in (1.31) in order to use (1.28) in Appendix B. We endow $\Theta_{T}$ with the following norm

$$
\left\|\left(\theta_{1}, \theta_{2}\right)\right\|_{\Theta_{T}}=\left\|\left(\theta_{1}, \theta_{2}\right)\right\|_{H^{2}(0, T)}+\left\|\left(\theta_{1}, \theta_{2}\right)\right\|_{L^{\infty}(0, T)}+\left\|\left(\dot{\theta}_{1}, \dot{\theta}_{2}\right)\right\|_{L^{\infty}(0, T)},
$$

the other spaces are endowed with their natural norms. The norm $\|\cdot\|_{\Theta_{T}}$ has been chosen so that we have the estimate $\left\|\left(\theta_{1}, \theta_{2}\right)\right\|_{L^{\infty}(0, T)}+\left\|\left(\dot{\theta}_{1}, \dot{\theta}_{2}\right)\right\|_{L^{\infty}(0, T)} \leq C\left\|\left(\theta_{1}, \theta_{2}\right)\right\|_{\Theta_{T}}$ where $C$ does not depend on $T$. Note that with the natural norm of $\Theta_{T}, C$ would depend on $T$.

\subsection{Main result}

The diffeomorphism $\Phi$. A classical difficulty in fluid-structure problems is that the fluid domain changes over time. The classical way to get rid of this difficulty is to use a change of variables on $\mathbf{u}$ and $p$ in order to bring the study back into a fixed domain. This procedure uses a diffeomorphism that we have to define properly. When the state of the structure depends only on a finite number of parameters, it is convenient to construct this diffeomorphism as an extension of the deformations of the structure into the fluid domain.

The diffeomorphism used is defined as an extension of the diffeomorphism $\mathbf{X}$ given for the structure. Hence, we need the extension operator defined below.

Lemma 1.7. There exists a linear extension operator $\mathcal{E}: \mathbf{W}^{3, \infty}\left(S_{0}\right) \rightarrow \mathbf{W}^{3, \infty}(\Omega) \cap \mathbf{H}_{0}^{1}(\Omega)$ such that for every $\mathbf{w} \in \mathbf{W}^{3, \infty}\left(S_{0}\right)$,

(i) $\quad \mathcal{E}(\mathbf{w})=\mathbf{w}$ in $S_{0}$

(ii) $\mathcal{E}(\mathbf{w})$ has support within $\Omega_{\varepsilon}=\{\mathbf{x} \in \Omega \mid \mathrm{d}(x, \partial \Omega)>\varepsilon\}$ for some $\varepsilon>0$ such that $\mathrm{d}\left(S\left(\theta_{1}, \theta_{2}\right), \partial \Omega\right)>2 \varepsilon$ for all $\left(\theta_{1}, \theta_{2}\right) \in \mathbb{D}_{\Theta}$,

(iii) $\quad\|\mathbf{w}\|_{\mathbf{w}^{3, \infty}(\Omega)} \leq C\|\mathbf{w}\|_{\mathbf{w}^{3, \infty}\left(S_{0}\right)}$, for some $C>0$.

Proof. Extension results are classical, we can for instance find an extension result for smooth domains in [20, Lemma 12.2]. We can get the result by multiplying the extension function of [20, Lemma 12.2] by a cut-off function in $\mathcal{D}\left(\Omega_{\varepsilon}\right)$. Note that the existence of $\varepsilon>0$ fulfilling (ii) is a consequence of Assumption (1.6).

Then we define the following function

$$
\mathbf{\Phi}\left(\theta_{1}, \theta_{2}, \mathbf{y}\right)=\mathbf{y}+\mathcal{E}\left(\mathbf{X}\left(\theta_{1}, \theta_{2}, .\right)-\mathrm{Id}\right)(\mathbf{y}), \quad \forall\left(\theta_{1}, \theta_{2}\right) \in \mathbb{D}_{\Theta}, \quad \forall \mathbf{y} \in \Omega
$$

where Id denotes the identity function. 
We have $\nabla \boldsymbol{\Phi}(0,0, \mathbf{y})=I$ for every $\mathbf{y} \in \Omega$, hence $\operatorname{det}(\nabla \boldsymbol{\Phi}(0,0, \mathbf{y}))=1$. Then for every $\left(\theta_{1}, \theta_{2}\right) \in \mathbb{D}_{\Theta}$ small enough, the function $\boldsymbol{\Phi}\left(\theta_{1}, \theta_{2},.\right)$ is a diffeomorphism close to the identity function. We denote $\boldsymbol{\Psi}\left(\theta_{1}, \theta_{2},.\right)$ the inverse diffeomorphism of $\boldsymbol{\Phi}\left(\theta_{1}, \theta_{2},.\right)$, i.e.

$$
\forall\left(\theta_{1}, \theta_{2}\right) \in \mathbb{D}_{\Theta}, \quad \forall \mathbf{y} \in \Omega, \quad \Psi\left(\theta_{1}, \theta_{2}, \mathbf{\Phi}\left(\theta_{1}, \theta_{2}, \mathbf{y}\right)\right)=\mathbf{y}
$$

We can prove that $\boldsymbol{\Phi}$ and $\boldsymbol{\Psi}$ belong to $\mathscr{C}^{\infty}\left(\mathbb{D}_{\Theta}, \mathbf{W}^{3, \infty}(\Omega)\right)$. These diffeomorphisms are represented in Fig. 2.

The properties of $\mathcal{E}$ imply that

$$
\text { for every }\left(\theta_{1}, \theta_{2}\right) \in \mathbb{D}_{\Theta}, \quad \boldsymbol{\Phi}\left(\theta_{1}, \theta_{2}, S_{0}\right)=S\left(\theta_{1}, \theta_{2}\right) \quad \text { and } \quad \forall \mathbf{y} \in \Omega \backslash \Omega_{\varepsilon}, \quad \boldsymbol{\Phi}\left(\theta_{1}, \theta_{2}, \mathbf{y}\right)=\mathbf{y} .
$$

The inflow boundary conditions. We use the following space to define the admissible boundary data on the inflow part of the boundary $\Gamma_{i}$,

$$
\mathbf{U}^{i}=\left\{\begin{array}{ccc}
\mathbf{u}^{i} \in \mathbf{H}^{3 / 2}\left(\Gamma_{\mathrm{i}}\right) \text { with } \mathbf{u}_{\mid \partial \Gamma_{\mathrm{i}}}^{i}=0, & \int_{0}^{1 / 4} \frac{\left|\partial_{y_{2}} u_{2}^{i}\left(y_{2}\right)\right|^{2}}{y_{2}} \mathrm{~d} y_{2}<+\infty \\
& \int_{3 / 4}^{1} \frac{\left|\partial_{y_{2}} u_{2}^{i}\left(y_{2}\right)\right|^{2}}{1-y_{2}} \mathrm{~d} y_{2}<+\infty
\end{array}\right\}
$$

The conditions with integrals in the definition of $\mathbf{U}^{i}$ are chosen to match the homogeneous boundary conditions on $\Gamma_{\mathrm{w}}$. We now state the following existence theorem.

Theorem 1.8 (Main result: Local existence in time of a solution). Let $T_{0}>0$, let $\mathbf{u}^{i} \in H^{1}\left(0, T_{0} ; \mathbf{U}^{i}\right)$, $\mathbf{u}_{0} \in \mathbf{H}^{1}\left(\mathscr{F}_{0}\right)$ and $\left(\omega_{1,0}, \omega_{2,0}\right) \in \mathbb{R}^{2}$ satisfying the compatibility conditions

$$
\begin{cases}\operatorname{div} \mathbf{u}_{0}=0 & \text { in } \mathscr{F}_{0} \\ \mathbf{u}_{0}(.)=\sum_{j=1}^{2} \omega_{j, 0} \partial_{\theta_{j}} \mathbf{X}(0,0, .) & \text { on } \partial S_{0} \\ \mathbf{u}_{0}=\mathbf{u}^{i}(0, .) & \text { on } \Gamma_{\mathrm{i}} \\ \mathbf{u}_{0}=0 & \text { on } \Gamma_{\mathrm{w}}\end{cases}
$$

Let $\mathbf{f}_{\mathscr{F}} \in L^{2}\left(0, T_{0} ; \mathbf{W}^{1, \infty}(\Omega)\right)$ and $\mathbf{f}_{\mathbf{s}} \in L^{2}\left(0, T_{0} ; \mathbb{R}^{2}\right)$. Then there exists a time $T \in\left(0, T_{0}\right]$ such that problem (1.24) admits a unique solution $\left(\mathbf{u}, p, \theta_{1}, \theta_{2}\right)$ with the following regularity

$$
\begin{aligned}
& \left(\theta_{1}, \theta_{2}\right) \in H^{2}\left(0, T ; \mathbb{D}_{\Theta}\right), \\
& \mathbf{u}\left(t, \boldsymbol{\Phi}\left(\theta_{1}(t), \theta_{2}(t), \mathbf{y}\right)\right) \in L^{2}\left(0, T ; \mathbf{H}_{\beta}^{2}\left(\mathscr{F}_{0}\right)\right) \cap \mathscr{C}^{0}\left([0, T] ; \mathbf{H}^{1}\left(\mathscr{F}_{0}\right)\right) \cap H^{1}\left(0, T ; \mathbf{L}^{2}\left(\mathscr{F}_{0}\right)\right), \\
& p\left(t, \boldsymbol{\Phi}\left(\theta_{1}(t), \theta_{2}(t), \mathbf{y}\right)\right) \in L^{2}\left(0, T ; H_{\beta}^{1}\left(\mathscr{F}_{0}\right)\right) .
\end{aligned}
$$

Moreover, we have the estimate

$$
\begin{aligned}
& \left\|\mathbf{u}\left(t, \boldsymbol{\Phi}\left(\theta_{1}(t), \theta_{2}(t), \mathbf{y}\right)\right)\right\|_{L^{2}\left(0, T ; \mathbf{H}_{\beta}^{2}\left(\mathscr{F}_{0}\right)\right) \cap \mathscr{C}^{0}\left([0, T] ; \mathbf{H}^{1}\left(\mathscr{F}_{0}\right)\right) \cap H^{1}\left(0, T ; \mathbf{L}^{2}\left(\mathscr{F}_{0}\right)\right)} \\
& \quad+\left\|p\left(t, \boldsymbol{\Phi}\left(\theta_{1}(t), \theta_{2}(t), \mathbf{y}\right)\right)\right\|_{L^{2}\left(0, T ; H_{\beta}^{1}\left(\mathscr{F}_{0}\right)\right)}+\left\|\left(\theta_{1}, \theta_{2}\right)\right\|_{H^{2}\left(0, T ; \mathbb{D}_{\Theta}\right)} \\
& \quad \leq C\left(\left\|\mathbf{u}_{0}\right\|_{\mathbf{H}^{1}\left(\mathscr{F}_{0}\right)}+\left|\omega_{1,0}\right|+\left|\omega_{2,0}\right|+\left\|\mathbf{f}_{\mathscr{F}}\right\|_{L^{2}\left(0, T_{0} ; \mathbf{L}^{2}\left(\mathscr{F}_{0}\right)\right)}+\left\|\mathbf{u}^{i}\right\|_{H^{1}\left(0, T_{0} ; \mathbf{H}^{3 / 2}\left(\Gamma_{\mathrm{i}}\right)\right)}+\left\|\mathbf{f}_{\mathbf{s}}\right\|_{L^{2}\left(0, T_{0} ; \mathbb{R}^{2}\right)}\right)
\end{aligned}
$$

The proof of Theorem 1.8 mainly follows the one in [10] and is presented in the rest of the present article. Remark 1.9. Note that we have the regularity $\mathbf{H}^{3 / 2+\varepsilon_{0}}$ for the fluid velocity as a consequence of (1.28).

Remark 1.10. The study in dimension three would require to adapt the functional framework with more intricate weighted Sobolev spaces (see [23]). An adaptation of Lemmas 1.5, 2.10 and estimate (B.32) would also be required.

Remark 1.11. In the present work, we do not study the global in time existence of solutions to the problem. This would be an interesting extension.

\subsection{Scientific context}

Fluid-structure interaction problems have been considered in several works. The structure is often rigid and immersed in an incompressible fluid [11, 15, 17, 18, 30, 32, 33] or a compressible one [7, 21, 22]. More complex structures have been studied for instance in $[12,28]$ where a plate immersed in an incompressible fluid has been considered or in $[4,19]$ where the authors studied the interaction between a $1 \mathrm{D}$ beam and a 2D fluid.

The interaction of an elastic structure with a compressible fluid has been studied in $[9,8]$ and with an incompressible fluid in [6].

Deforming structures that have a given deformation have been considered to model fish-like swimmers $[24,29]$. However, they do not fit our framework since, in the present study, the deformations of the structure fulfill an ODE. 
The case of a deformable structure depending on a finite numbers of degrees of freedom can be found in [10]. The model of the structure approximates the linear elasticity equations and it depends only on a finite number of degrees of freedom. However, to the best of our knowledge, besides the rigid solids, we have not found any work dealing with a structure depending naturally on a finite number of degrees of freedom fulfilling an equation. In that sense, the modelling proposed in the present article is original.

Moreover, additional difficulties are induced by the corners on $\partial \Omega$, more information can be found in [23, 25].

\subsection{Outline of the paper}

In the next sections, we give the proof of the main result. In Section 2, we study the linearized problem in the fixed domain $\mathscr{F}_{0}$ and prove existence of strong solutions. We first discard the boundary terms to ease the study. The full linearized problem is then treated by the use of liftings. In Section 3, we prove local existence of strong solutions to the nonlinear system. The proof is first conducted in the fixed domain by using the results on the linearized system and a fixed point argument. We then obtain the main result with a change of variables. In Appendix A, we prove the fact that the proposed diffeomorphism $\mathbf{X}^{b}$ of Section 1.1.1 fulfils the modelling assumptions. Finally, the proof of the estimates of the nonlinear terms can be found in Appendix B.

\section{Existence of solution to the linearized problem}

In this section we study the linearization of problem (1.24) around the null state, first with only source terms $\mathbf{f}$ and $\mathbf{s}$ and then with all source terms and boundary data. These equations are written in the fixed domain $\mathscr{F}_{0}$ using a change of variables explained in Section 3.1. In the sequel, $(\tilde{\mathbf{u}}, \tilde{p})$ denotes the velocity and the pressure of the fluid in the fixed domain $\mathscr{F}_{0}$. We denote $T>0$ the considered final time.

\subsection{Linearized problem with nonhomogeneous source terms}

Let us study the following problem

$$
\begin{cases}\frac{\partial \tilde{\mathbf{u}}}{\partial t}-\nu \Delta \tilde{\mathbf{u}}+\nabla \tilde{p}=\mathbf{f} & \text { in }(0, T) \times \mathscr{F}_{0}, \\
\operatorname{div} \tilde{\mathbf{u}}=0 & \text { in }(0, T) \times \mathscr{F}_{0}, \\
\tilde{\mathbf{u}}=\dot{\theta}_{1} \partial_{\theta_{1}} \boldsymbol{\Phi}(0,0, .)+\dot{\theta}_{2} \partial_{\theta_{2}} \mathbf{\Phi}(0,0, .) & \text { on }(0, T) \times \partial S_{0} \\
\tilde{\mathbf{u}}=0 & \text { on }(0, T) \times \Gamma_{\mathrm{i}}, \\
\tilde{\mathbf{u}}=0 & \text { on }(0, T) \times \Gamma_{\mathrm{w}}, \\
\sigma_{F}(\tilde{\mathbf{u}}, \tilde{p}) \mathbf{n}=0 & \text { on }(0, T) \times \Gamma_{\mathrm{N}}, \\
\tilde{\mathbf{u}}(0, .)=\mathbf{u}_{0}(.) & \text { in } \mathscr{F}_{0}, \\
\mathcal{M}_{0,0}\left(\begin{array}{c}
\ddot{\theta}_{1} \\
\ddot{\theta}_{2}
\end{array}\right)=\left(\int_{\partial S_{0}}\left[\tilde{p} I-\nu\left(\nabla \tilde{\mathbf{u}}+(\nabla \tilde{\mathbf{u}})^{T}\right)\right] \mathbf{n}_{0} \cdot \partial_{\theta_{1}} \mathbf{\Phi}\left(0,0, \gamma_{y}\right) \mathrm{d} \gamma_{y}\right) & \text { on }(0, T), \\
\left.\int_{\partial S_{0}}\left[\tilde{p} I-\nu\left(\nabla \tilde{\mathbf{u}}+(\nabla \tilde{\mathbf{u}})^{T}\right)\right] \mathbf{n}_{0} \cdot \partial_{\theta_{2}} \boldsymbol{\Phi}\left(0,0, \gamma_{y}\right) \mathrm{d} \gamma_{y}\right) & \mathbf{s}\end{cases}
$$

where the unkwnows are $\left(\tilde{\mathbf{u}}, \tilde{p}, \theta_{1}, \theta_{2}\right)$ and the source terms are $(\mathbf{f}, \mathbf{s}) \in L^{2}\left(0, T ; \mathbf{L}^{2}\left(\mathscr{F}_{0}\right)\right) \times L^{2}\left(0, T ; \mathbb{R}^{2}\right)$. We will show later that this system corresponds to the linearization of the nonlinear problem (1.24) transported in the fixed initial configuration $\mathscr{F}_{0}$.

Remark 2.1. The state $\left(\tilde{\mathbf{u}}, \tilde{p}, \theta_{1}, \theta_{2}\right)$ of problem $(2.1)$ can be reduced to $\left(\tilde{\mathbf{u}}, \tilde{p}, \dot{\theta}_{1}, \dot{\theta}_{2}\right)$. Considering the velocity of the structure instead of its position is sufficient to solve (2.1). However, we prefer to consider the full state $\left(\tilde{\mathbf{u}}, \tilde{p}, \theta_{1}, \theta_{2}\right)$, as it is useful in the sequel to deal with the nonlinear problem.

Let us fix an arbitrary time $T_{0}>0$, e.g. $T_{0}=1$. We want to prove the following result.

Proposition 2.2. There exists a constant $C>0$ such that for all $T \in\left(0, T_{0}\right)$, $C$ does not depend on $T$, for all $\mathbf{u}_{0} \in \mathbf{H}^{1}\left(\mathscr{F}_{0}\right)$ and $\left(\omega_{1,0}, \omega_{2,0}\right) \in \mathbb{R}^{2}$ satisfying the compatibility conditions $(1.41)$ (with $\left.\mathbf{u}^{i}=0\right)$ and every $(\mathbf{f}, \mathbf{s}) \in \mathbb{F}_{T} \times \mathbb{S}_{T}$, problem (2.1) admits a unique solution

$$
\left(\tilde{\mathbf{u}}, \tilde{p}, \theta_{1}, \theta_{2}\right) \in \mathbb{U}_{T} \times \mathbb{P}_{T} \times \Theta_{T}
$$

Moreover, the following estimate holds

$$
\|\tilde{\mathbf{u}}\|_{\mathbb{U}_{T}}+\|\tilde{p}\|_{\mathbb{P}_{T}}+\left\|\left(\theta_{1}, \theta_{2}\right)\right\|_{\Theta_{T}} \leq C\left(\left\|\mathbf{u}_{0}\right\|_{\mathbf{H}^{1}\left(\mathscr{F}_{0}\right)}+\left|\omega_{1,0}\right|+\left|\omega_{2,0}\right|+\|\mathbf{f}\|_{\mathbb{F}_{T}}+\|\mathbf{s}\|_{\mathbb{S}_{T}}\right) .
$$


In order to prove Proposition 2.2, we will study problem (2.1) under its semigroup formulation. Let us define the space

$$
\mathbb{H}=\left\{\begin{array}{ll}
\left(\tilde{\mathbf{u}}, \theta_{1}, \theta_{2}, \omega_{1}, \omega_{2}\right) \in \mathbf{L}^{2}\left(\mathscr{F}_{0}\right) \times \mathbb{R}^{4}, & \operatorname{div} \tilde{\mathbf{u}}=0 \text { in } \mathscr{F}_{0}, \quad \tilde{\mathbf{u}} \cdot \mathbf{n}=0 \text { on } \Gamma_{\mathrm{D}}, \\
& \tilde{\mathbf{u}} \cdot \mathbf{n}_{0}=\sum_{j} \omega_{j} \partial_{\theta_{j}} \mathbf{\Phi}(0,0, .) \cdot \mathbf{n}_{0} \text { on } \partial S_{0}
\end{array}\right\}
$$

where $\mathbf{n}_{0}$ is the unit outward normal to the fluid domain $\mathscr{F}_{0}$. The space $\mathbb{H}$ is endowed with the scalar product $(., .)_{0}$ of $\mathbf{L}^{2}\left(\mathscr{F}_{0}\right) \times \mathbb{R}^{4}$ defined by

$$
\left(\left(\tilde{\mathbf{u}}^{a}, \theta_{1}^{a}, \theta_{2}^{a}, \omega_{1}^{a}, \omega_{2}^{a}\right),\left(\tilde{\mathbf{u}}^{b}, \theta_{1}^{b}, \theta_{2}^{b}, \omega_{1}^{b}, \omega_{2}^{b}\right)\right)_{0}=\int_{\mathscr{F}_{0}} \tilde{\mathbf{u}}^{a} \cdot \tilde{\mathbf{u}}^{b} \mathrm{~d} \mathbf{y}+\left(\begin{array}{ll}
\theta_{1}^{a} & \theta_{2}^{a}
\end{array}\right)\left(\begin{array}{c}
\theta_{1}^{b} \\
\theta_{2}^{b}
\end{array}\right)+\left(\begin{array}{ll}
\omega_{1}^{a} & \omega_{2}^{a}
\end{array}\right) \mathcal{M}_{0,0}\left(\begin{array}{c}
\omega_{1}^{b} \\
\omega_{2}^{b}
\end{array}\right)
$$

where $\mathcal{M}_{0,0}$ is the matrix defined in (1.16) with $\theta_{1}=\theta_{2}=0$. We also define

$$
\mathbb{V}=\left\{\begin{array}{ll}
\left(\tilde{\mathbf{u}}, \theta_{1}, \theta_{2}, \omega_{1}, \omega_{2}\right) \in \mathbf{H}^{1}\left(\mathscr{F}_{0}\right) \times \mathbb{R}^{4}, & \operatorname{div} \tilde{\mathbf{u}}=0 \text { in } \mathscr{F} 0, \quad \tilde{\mathbf{u}}=0 \text { on } \Gamma_{\mathrm{D}}, \\
& \tilde{\mathbf{u}}=\sum_{j} \omega_{j} \partial_{\theta_{j}} \mathbf{\Phi}(0,0, .) \text { on } \partial S_{0}
\end{array}\right\}
$$

endowed with the scalar product $(., .)_{1}$ of $\mathbf{H}^{1}\left(\mathscr{F}_{0}\right) \times \mathbb{R}^{4}$ defined by

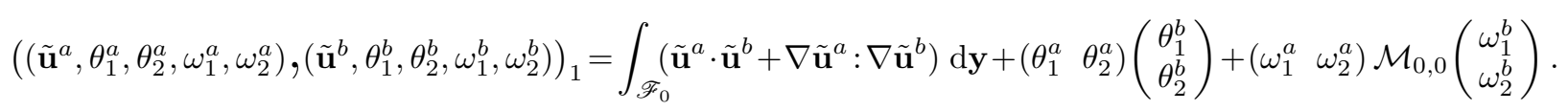

In the sequel, we denote $\left(f_{j}\right)_{j=1,2}=\left(\begin{array}{c}f_{1} \\ f_{2}\end{array}\right)$.

Lemma 2.3. The orthogonal space to $\mathbb{H}$ with respect to the scalar product $(., .)_{0}$ is

$$
(\mathbb{H})^{\perp}=\left\{\left(\nabla p, 0,0,-\mathcal{M}_{0,0}^{-1}\left(\int_{\partial S_{0}} p \mathbf{n}_{0} \cdot \partial_{\theta_{j}} \mathbf{\Phi}\left(0,0, \gamma_{y}\right) \mathrm{d} \gamma_{y}\right)_{j=1,2}\right) \text { with } p \in H^{1}\left(\mathscr{F}_{0}\right), p=0 \text { on } \Gamma_{\mathrm{N}}\right\} .
$$

Proof. Let $\left(\tilde{\mathbf{u}}^{a}, \theta_{1}^{a}, \theta_{2}^{a}, \omega_{1}^{a}, \omega_{2}^{a}\right) \in \mathbf{L}^{2}\left(\mathscr{F}_{0}\right) \times \mathbb{R}^{4}$ such that for every $\left(\tilde{\mathbf{u}}^{b}, \theta_{1}^{b}, \theta_{2}^{b}, \omega_{1}^{b}, \omega_{2}^{b}\right) \in \mathbb{H}$,

$$
\left(\left(\tilde{\mathbf{u}}^{a}, \theta_{1}^{a}, \theta_{2}^{a}, \omega_{1}^{a}, \omega_{2}^{a}\right),\left(\tilde{\mathbf{u}}^{b}, \theta_{1}^{b}, \theta_{2}^{b}, \omega_{1}^{b}, \omega_{2}^{b}\right)\right)_{0}=0 .
$$

By taking $\tilde{\mathbf{u}}^{b}=0$ and $\omega_{1}^{b}=\omega_{2}^{b}=0$, we easily obtain $\theta_{1}^{a}=\theta_{2}^{a}=0$. With $\omega_{1}^{b}=\omega_{2}^{b}=0$, we also get

$$
\int_{\mathscr{F}_{0}} \tilde{\mathbf{u}}^{a} \cdot \tilde{\mathbf{u}}^{b} \mathrm{~d} \mathbf{y}=0, \quad \forall \tilde{\mathbf{u}}^{b} \in \mathbf{L}^{2}\left(\mathscr{F}_{0}\right) \text { such that } \operatorname{div} \tilde{\mathbf{u}}^{b}=0 \text { in } \mathscr{F}_{0} \text { and } \tilde{\mathbf{u}}^{b} \cdot \mathbf{n}_{0}=0 \text { on } \Gamma_{\mathrm{D}} \cup \partial S_{0},
$$

which implies, according to $[25$, Lemma 2.2$], \tilde{\mathbf{u}}^{a}=\nabla p$, where $p \in H^{1}\left(\mathscr{F}_{0}\right)$ and $p=0$ on $\Gamma_{\mathrm{N}}$. Now,

$$
\int_{\mathscr{F}_{0}} \nabla p \cdot \tilde{\mathbf{u}}^{b} \mathrm{~d} \mathbf{y}+\sum_{j, k} \omega_{j}^{a} \omega_{k}^{b}\left(\partial_{\theta_{j}} \mathbf{X}(0,0, .), \partial_{\theta_{k}} \mathbf{X}(0,0, .)\right)_{S}=0
$$

becomes with the divergence formula and the compatibility condition in (2.3)

$$
\sum_{j} \omega_{j}^{b} \int_{\partial S_{0}} p \mathbf{n}_{0} \cdot \partial_{\theta_{j}} \mathbf{\Phi}\left(0,0, \gamma_{y}\right) \mathrm{d} \gamma_{y}+\sum_{j, k} \omega_{j}^{a} \omega_{k}^{b}\left(\partial_{\theta_{j}} \mathbf{X}(0,0, .), \partial_{\theta_{k}} \mathbf{X}(0,0, .)\right)_{S}=0
$$

then

$$
\int_{\partial S_{0}} p \mathbf{n}_{0} \cdot \partial_{\theta_{j}} \mathbf{\Phi}\left(0,0, \gamma_{y}\right) \mathrm{d} \gamma_{y}+\sum_{k} \omega_{k}^{a}\left(\partial_{\theta_{j}} \mathbf{X}(0,0, .), \partial_{\theta_{k}} \mathbf{X}(0,0, .)\right)_{S}=0,
$$

which yields a first inclusion. The converse inclusion is obtained via an integration by parts.

We define the operator $(A, D(A))$ on $\mathbb{H}$ as

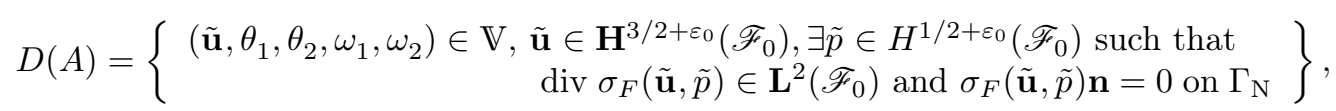

where $\varepsilon_{0}$ is introduced in Lemma 1.5, and

$$
A\left(\begin{array}{c}
\tilde{\mathbf{u}} \\
\theta_{1} \\
\theta_{2} \\
\omega_{1} \\
\omega_{2}
\end{array}\right)=\Pi_{\mathbb{H}}\left(\begin{array}{l}
\operatorname{div} \sigma_{F}(\tilde{\mathbf{u}}, \tilde{p}) \\
\omega_{1} \\
\omega_{2} \\
\mathcal{M}_{0,0}^{-1}\left(\int_{\partial S_{0}}-\sigma_{F}(\tilde{\mathbf{u}}, \tilde{p}) \mathbf{n}_{0} \cdot \partial_{\theta_{j}} \boldsymbol{\Phi}\left(0,0, \gamma_{y}\right) \mathrm{d} \gamma_{y}\right)_{j=1,2}
\end{array}\right)
$$

where $\Pi_{\mathbb{H}}$ is the orthogonal projector from $\mathbf{L}^{2}\left(\mathscr{F}_{0}\right) \times \mathbb{R}^{4}$ onto $\mathbb{H}$ with respect to $(., .)_{0}$. 
Remark 2.4. The use of $\tilde{p}$ in the definition of $(A, D(A))$ is useful to guarantee that $\operatorname{div} \sigma_{F}(\tilde{\mathbf{u}}, \tilde{p})$ belongs to $\mathbf{L}^{2}\left(\mathscr{F}_{0}\right)$ and then that the application of $\Pi_{\mathbb{H}}$ in the right hand-side of (2.6) makes sense.

Lemma 2.5. The operator $A$ is uniquely defined.

Proof. Let $\left(\tilde{\mathbf{u}}, \theta_{1}, \theta_{2}, \omega_{1}, \omega_{2}\right) \in D(A)$ and consider two functions $p, q \in H^{1 / 2+\varepsilon_{0}}\left(\mathscr{F}_{0}\right)$ satisfying the conditions appearing into the definition of $D(A)$. Then, $\operatorname{div} \sigma_{F}(0, p-q)=-\nabla(p-q) \in \mathbf{L}^{2}\left(\mathscr{F}_{0}\right)$ implies $p-q \in H^{1}\left(\mathscr{F}_{0}\right)$, and $\sigma_{F}(0, p-q) \mathbf{n}=0$ on $\Gamma_{\mathrm{N}}$ implies $p-q=0$ on $\Gamma_{\mathrm{N}}$.

Now,

$$
\begin{aligned}
\left(\begin{array}{l}
\operatorname{div} \sigma_{F}(\tilde{\mathbf{u}}, p) \\
\omega_{1} \\
\omega_{2} \\
\mathcal{M}_{0,0}^{-1}\left(\int_{\partial S_{0}}-\sigma_{F}(\tilde{\mathbf{u}}, p) \mathbf{n}_{0} \cdot \partial_{\theta_{j}} \boldsymbol{\Phi}\left(0,0, \gamma_{y}\right) \mathrm{d} \gamma_{y}\right)_{j=1,2}
\end{array}\right)-\left(\begin{array}{l}
\operatorname{div} \sigma_{F}(\tilde{\mathbf{u}}, q) \\
\omega_{1} \\
\omega_{2} \\
\mathcal{M}_{0,0}^{-1}\left(\int_{\partial S_{0}}-\sigma_{F}(\tilde{\mathbf{u}}, q) \mathbf{n}_{0} \cdot \partial_{\theta_{j}} \boldsymbol{\Phi}\left(0,0, \gamma_{y}\right) \mathrm{d} \gamma_{y}\right)_{j=1,2}
\end{array}\right) \\
=\left(\begin{array}{l}
\nabla(p-q) \\
0 \\
0 \\
-\mathcal{M}_{0,0}^{-1}\left(\int_{\partial S_{0}}(p-q) \mathbf{n}_{0} \cdot \partial_{\theta_{j}} \boldsymbol{\Phi}\left(0,0, \gamma_{y}\right) \mathrm{d} \gamma_{y}\right)_{j=1,2}
\end{array}\right),
\end{aligned}
$$

which belongs to $\mathrm{H}^{\perp}$ according to Lemma 2.3. Therefore $A$ is uniquely defined.

Before going further, let us point out that $D(A)$ can be characterized as follows.

Lemma 2.6. We have

$$
D(A)=\left\{\begin{aligned}
&\left(\tilde{\mathbf{u}}, \theta_{1}, \theta_{2}, \omega_{1}, \omega_{2}\right) \in \mathbb{V}, \tilde{\mathbf{u}} \in \mathbf{H}_{\beta}^{2}\left(\mathscr{F}_{0}\right), \exists \tilde{p} \in H_{\beta}^{1}\left(\mathscr{F}_{0}\right) \text { such that } \\
& \operatorname{div} \sigma_{F}(\tilde{\mathbf{u}}, \tilde{p}) \in \mathbf{L}^{2}\left(\mathscr{F}_{0}\right) \text { and } \sigma_{F}(\tilde{\mathbf{u}}, \tilde{p}) \mathbf{n}=0 \text { on } \Gamma_{\mathrm{N}}
\end{aligned}\right\} .
$$

Proof. Assume that $\left(\tilde{\mathbf{u}}, \theta_{1}, \theta_{2}, \omega_{1}, \omega_{2}\right)$ belongs to $D(A)$ given by $(2.5)$. Then $(\tilde{\mathbf{u}}, \tilde{p})$ satisfies

$$
\begin{cases}\operatorname{div} \sigma_{F}(\tilde{\mathbf{u}}, \tilde{p}) & \in \mathbf{L}^{2}\left(\mathscr{F}_{0}\right), \\ \operatorname{div} \tilde{\mathbf{u}}=0 & \text { in } \mathscr{F}_{0} \\ \tilde{\mathbf{u}}=\sum_{j} \omega_{j} \partial_{\theta_{j}} \mathbf{\Phi}(0,0, .) & \text { on } \partial S_{0} \\ \tilde{\mathbf{u}}=0 & \text { on } \Gamma_{\mathrm{D}} \\ \sigma_{F}(\tilde{\mathbf{u}}, \tilde{p}) \mathbf{n}=0 & \text { on } \Gamma_{\mathrm{N}}\end{cases}
$$

According to [25, Theorem 2.16], there exists $\mathbf{v}_{s} \in \mathbf{H}^{2}\left(\mathscr{F}_{0}\right)$ such that

$$
\begin{cases}\operatorname{div} \sigma_{F}\left(\mathbf{v}_{s}, 0\right)=0 & \text { in } \mathscr{F}_{0} \\ \operatorname{div} \mathbf{v}_{s}=0 & \text { in } \mathscr{F}_{0} \\ \mathbf{v}_{s}=\sum_{j} \omega_{j} \partial_{\theta_{j}} \boldsymbol{\Phi}(0,0, .) & \text { on } \partial S_{0} \\ \mathbf{v}_{s}=0 & \text { on } \Gamma_{\mathrm{D}} \\ \sigma_{F}\left(\mathbf{v}_{s}, 0\right) \mathbf{n}=0 & \text { on } \Gamma_{\mathrm{N}}\end{cases}
$$

Let $\mathbf{f}=-\operatorname{div} \sigma_{F}(\tilde{\mathbf{u}}, \tilde{p}) \in \mathbf{L}^{2}\left(\mathscr{F}_{0}\right)$. Then $\left(\tilde{\mathbf{u}}-\mathbf{v}_{s}, \tilde{p}\right)$ satisfies

$$
\begin{cases}-\operatorname{div} \sigma_{F}\left(\tilde{\mathbf{u}}-\mathbf{v}_{s}, \tilde{p}\right)=\mathbf{f} & \text { in } \mathscr{F}_{0} \\ \operatorname{div}\left(\tilde{\mathbf{u}}-\mathbf{v}_{s}\right)=0 & \text { in } \mathscr{F}_{0} \\ \tilde{\mathbf{u}}-\mathbf{v}_{s}=0 & \text { on } \Gamma_{\mathrm{D}} \cup \partial S_{0} \\ \sigma_{F}\left(\tilde{\mathbf{u}}-\mathbf{v}_{s}, \tilde{p}\right) \mathbf{n}=0 & \text { on } \Gamma_{\mathrm{N}}\end{cases}
$$

According to Lemma 1.5, $\tilde{\mathbf{u}}-\mathbf{v}_{s} \in \mathbf{H}_{\beta}^{2}\left(\mathscr{F}_{0}\right) \cap \mathbf{H}^{3 / 2+\varepsilon_{0}}\left(\mathscr{F}_{0}\right), \tilde{p} \in H_{\beta}^{1}\left(\mathscr{F}_{0}\right) \cap \mathbf{H}^{1 / 2+\varepsilon_{0}}\left(\mathscr{F}_{0}\right)$. This ends the proof.

We define the bilinear form $\mathrm{a}_{1}$ on $\mathbb{V} \times \mathbb{V}$ for every $\left(\tilde{\mathbf{u}}^{a}, \theta_{1}^{a}, \theta_{2}^{a}, \omega_{1}^{a}, \omega_{2}^{a}\right)$ and $\left(\tilde{\mathbf{u}}^{b}, \theta_{1}^{b}, \theta_{2}^{b}, \omega_{1}^{b}, \omega_{2}^{b}\right)$ in $\mathbb{V}$ by

$$
\mathrm{a}_{1}\left(\left(\tilde{\mathbf{u}}^{a}, \theta_{1}^{a}, \theta_{2}^{a}, \omega_{1}^{a}, \omega_{2}^{a}\right),\left(\tilde{\mathbf{u}}^{b}, \theta_{1}^{b}, \theta_{2}^{b}, \omega_{1}^{b}, \omega_{2}^{b}\right)\right)=\frac{\nu}{2} \int_{\mathscr{F}_{0}}\left(\nabla \tilde{\mathbf{u}}^{a}+\left(\nabla \tilde{\mathbf{u}}^{a}\right)^{T}\right):\left(\nabla \tilde{\mathbf{u}}^{b}+\left(\nabla \tilde{\mathbf{u}}^{b}\right)^{T}\right) \mathrm{d} \mathbf{y}
$$

We define the operator $\left(A_{1}, D(A)\right)$ on $\mathbb{H}$ by

$$
D\left(A_{1}\right)=\left\{\mathbf{z} \in \mathbb{V} \text { with } \tilde{\mathbf{z}} \mapsto \mathrm{a}_{1}(\mathbf{z}, \tilde{\mathbf{z}}) \text { is } \mathbb{H}-\text { continuous }\right\},
$$

and

$$
\forall \mathbf{z} \in D\left(A_{1}\right), \quad \forall \tilde{\mathbf{z}} \in \mathbb{V}, \quad\left(A_{1} \mathbf{z}, \tilde{\mathbf{z}}\right)_{0}=-\mathrm{a}_{1}(\mathbf{z}, \tilde{\mathbf{z}})
$$


Lemma 2.7. We have

$$
D\left(A_{1}\right)=D(A)
$$

and

$$
A_{1}\left(\begin{array}{c}
\tilde{\mathbf{u}} \\
\theta_{1} \\
\theta_{2} \\
\omega_{1} \\
\omega_{2}
\end{array}\right)=\Pi_{\mathbb{H}}\left(\begin{array}{l}
\operatorname{div} \sigma_{F}(\tilde{\mathbf{u}}, \tilde{p}) \\
0 \\
0 \\
\mathcal{M}_{0,0}^{-1}\left(\int_{\partial S_{0}}-\sigma_{F}(\tilde{\mathbf{u}}, \tilde{p}) \mathbf{n}_{0} \cdot \partial_{\theta_{j}} \boldsymbol{\Phi}\left(0,0, \gamma_{y}\right) \mathrm{d} \gamma_{y}\right)_{j=1,2}
\end{array}\right) .
$$

Proof. The inclusion $D(A) \subset D\left(A_{1}\right)$ comes easily. Moreover, for every $\mathbf{z}=\left(\tilde{\mathbf{u}}, \theta_{1}, \theta_{2}, \omega_{1}, \omega_{2}\right) \in D(A)$, an integration by parts yields

$$
\forall \tilde{\mathbf{z}} \in \mathbb{V}, \quad\left(A_{1} \mathbf{z}, \tilde{\mathbf{z}}\right)_{0}=\left(\left(\begin{array}{l}
\operatorname{div} \sigma_{F}(\tilde{\mathbf{u}}, \tilde{p}) \\
0 \\
0 \\
\mathcal{M}_{0,0}^{-1}\left(\int_{\partial S_{0}}-\sigma_{F}(\tilde{\mathbf{u}}, \tilde{p}) \mathbf{n}_{0} \cdot \partial_{\theta_{j}} \mathbf{\Phi}\left(0,0, \gamma_{y}\right) \mathrm{d} \gamma_{y}\right)_{j=1,2}
\end{array}\right), \tilde{\mathbf{z}}\right) .
$$

Let us now prove the reverse inclusion $D\left(A_{1}\right) \subset D(A)$. Let $\mathbf{z}=\left(\tilde{\mathbf{u}}, \theta_{1}, \theta_{2}, \omega_{1}, \omega_{2}\right) \in D\left(A_{1}\right)$. According to Riesz representation theorem, there exists $\mathbf{f} \in \mathbb{H}$ such that

$$
\forall \tilde{\mathbf{z}} \in \mathbb{V}, \quad \mathrm{a}_{1}(\mathbf{z}, \tilde{\mathbf{z}})=(\mathbf{f}, \tilde{\mathbf{z}})_{0} .
$$

We write $\mathbf{f}=\left(\mathbf{f}_{\mathbf{u}}, \mathbf{f}_{\theta_{1}}, \mathbf{f}_{\theta_{2}}, \mathbf{f}_{\omega_{1}}, \mathbf{f}_{\omega_{2}}\right)$. For $\widetilde{\mathbf{v}} \in \mathcal{D}_{\text {div }}=\left\{\mathbf{u} \in\left(\mathscr{C}_{c}^{\infty}\left(\mathscr{F}_{0}\right)\right)^{2}\right.$ with $\left.\operatorname{div} \mathbf{u}=0\right\}$, we know that $(\widetilde{\mathbf{v}}, 0,0,0,0)$ belongs to $\mathbb{V}$, and an integration by parts yields

$$
\mathrm{a}_{1}(\mathbf{z},(\widetilde{\mathbf{v}}, 0,0,0,0))=\int_{\mathscr{F}_{0}}\left(-\operatorname{div} \sigma_{F}(\tilde{\mathbf{u}}, 0)\right) \cdot \widetilde{\mathbf{v}} \mathrm{d} \mathbf{y}=\int_{\mathscr{F}_{0}} \mathbf{f}_{\mathbf{u}} \cdot \widetilde{\mathbf{v}} \mathrm{d} \mathbf{y} .
$$

Then, according to [31, Lemma 2.2.2], there exists $\widehat{q} \in L^{2}\left(\mathscr{F}_{0}\right)$ such that

$$
-\operatorname{div} \sigma_{F}(\tilde{\mathbf{u}}, \widehat{q})=\mathbf{f}_{\mathbf{u}} \text { in } \mathscr{F}_{0},
$$

and thus $\operatorname{div} \sigma_{F}(\tilde{\mathbf{u}}, \widehat{q})$ belongs to $\mathbf{L}^{2}\left(\mathscr{F}_{0}\right)$, which gives a meaning to $\sigma_{F}(\tilde{\mathbf{u}}, \widehat{q}) \mathbf{n}_{0}$ on $\partial \mathscr{F}_{0}$.

Now, let us prove that $\sigma_{F}(\tilde{\mathbf{u}}, \widehat{q}) \mathbf{n}$ is constant along $\Gamma_{\mathrm{N}}$. Let $\mathbf{g} \in\left(\mathscr{C}_{c}^{\infty}\left(\Gamma_{\mathrm{N}}\right)\right)^{2}$ fulfilling $\int_{\Gamma_{\mathrm{N}}} \mathbf{g} \cdot \mathbf{n} \mathrm{d} \gamma_{y}=0$. According to [14, Theorem IV.1.1], there exists $\mathbf{v}_{\mathbf{g}} \in \mathbf{H}^{1}\left(\mathscr{F}_{0}\right)$ satisfying

$$
\left\{\begin{array}{l}
\operatorname{div} \mathbf{v}_{\mathbf{g}}=0 \text { in } \mathscr{F}_{0} \\
\mathbf{v}_{\mathbf{g}}=0 \text { on } \Gamma_{\mathrm{D}} \cup \partial S_{0} \\
\mathbf{v}_{\mathbf{g}}=\mathbf{g} \text { on } \Gamma_{\mathrm{N}}
\end{array}\right.
$$

We know that $\left(\mathbf{v}_{\mathbf{g}}, 0,0,0,0\right)$ belongs to $\mathbb{V}$. An integration by parts yields

$$
\mathrm{a}_{1}\left(\mathbf{z},\left(\mathbf{v}_{\mathbf{g}}, 0,0,0,0\right)\right)=\int_{\mathscr{F}_{0}}\left(-\operatorname{div} \sigma_{F}(\tilde{\mathbf{u}}, \widehat{q})\right) \cdot \mathbf{v}_{\mathbf{g}} \mathrm{d} \mathbf{y}+\int_{\Gamma_{\mathrm{N}}} \sigma_{F}(\tilde{\mathbf{u}}, \widehat{q}) \mathbf{n} \cdot \mathbf{g} \mathrm{d} \gamma_{y}=\int_{\mathscr{F}_{0}} \mathbf{f}_{\mathbf{u}} \cdot \mathbf{v}_{\mathbf{g}} \mathrm{d} \mathbf{y},
$$

and with (2.7) we get

$$
\int_{\Gamma_{\mathrm{N}}} \sigma_{F}(\tilde{\mathbf{u}}, \widehat{q}) \mathbf{n} \cdot \mathbf{g} \mathrm{d} \gamma_{y}=0
$$

The previous equality holds for every $\mathbf{g} \in\left(\mathscr{C}_{c}^{\infty}\left(\Gamma_{\mathrm{N}}\right)\right)^{2}$ fulfilling $\int_{\Gamma_{\mathrm{N}}} \mathbf{g} \cdot \mathbf{n} \mathrm{d} \gamma_{y}=0$, then there exists a constant $c$ such that $\sigma_{F}(\tilde{\mathbf{u}}, \widehat{q}) \mathbf{n}=c \mathbf{n}$ on $\Gamma_{\mathrm{N}}$.

Let $q=\widehat{q}-c \in L^{2}\left(\mathscr{F}_{0}\right)$, we have $\operatorname{div} \sigma_{F}(\tilde{\mathbf{u}}, q)=\operatorname{div} \sigma_{F}(\tilde{\mathbf{u}}, \widehat{q})$ and $\sigma_{F}(\tilde{\mathbf{u}}, q) \mathbf{n}=0$ on $\Gamma_{\mathrm{N}}$. Moreover, $(\tilde{\mathbf{u}}, q)$ satisfies

$$
\begin{cases}\operatorname{div} \sigma_{F}(\tilde{\mathbf{u}}, q) & \in \mathbf{L}^{2}\left(\mathscr{F}_{0}\right) \\ \operatorname{div} \tilde{\mathbf{u}}=0 & \text { in } \mathscr{F}_{0} \\ \tilde{\mathbf{u}}=0 & \text { on } \Gamma_{\mathrm{D}} \\ \tilde{\mathbf{u}}=\sum_{j} \omega_{j} \partial_{\theta_{j}} \boldsymbol{\Phi}(0,0, .) & \text { on } \partial S_{0} \\ \sigma_{F}(\tilde{\mathbf{u}}, q) \mathbf{n}=0 & \text { on } \Gamma_{\mathrm{N}}\end{cases}
$$

We finish this proof with a lifting of the boundary datum on $\partial S_{0}[25$, Theorem 2.16] and Lemma 1.5. We get $D\left(A_{1}\right) \subset D(A)$, thus concluding the proof of Lemma 2.7 . 
The key point of this section is the following lemma.

Lemma 2.8. The operator $A$ generates an analytic semigroup on $\mathbb{H}$. Moreover, for $\lambda \in \mathbb{R}$ large enough, $\lambda I-A$ is positive and $D\left((\lambda I-A)^{1 / 2}\right)=\mathbb{V}$.

Proof. We first prove the properties of Lemma 2.8 on the self-adjoint operator $A_{1}$ and then we extend it to $A$ with a perturbation argument.

The bilinear form $\mathrm{a}_{1}$ is symmetric and according to Korn's inequality [13, p. 110], there exists $c>0$ such that

$$
\forall\left(\tilde{\mathbf{u}}, \theta_{1}, \theta_{2}, \omega_{1}, \omega_{2}\right) \in \mathbb{V}, \mathrm{a}_{1}\left(\left(\tilde{\mathbf{u}}, \theta_{1}, \theta_{2}, \omega_{1}, \omega_{2}\right),\left(\tilde{\mathbf{u}}, \theta_{1}, \theta_{2}, \omega_{1}, \omega_{2}\right)\right)+\frac{\nu}{2}\|\tilde{\mathbf{u}}\|_{\mathbf{L}^{2}\left(\mathscr{F}_{0}\right)}^{2} \geq c\|\tilde{\mathbf{u}}\|_{\mathbf{H}^{1}\left(\mathscr{F}_{0}\right)}^{2},
$$

so that,

$$
\begin{aligned}
& \forall\left(\tilde{\mathbf{u}}, \theta_{1}, \theta_{2}, \omega_{1}, \omega_{2}\right) \in \mathbb{V} \\
& \mathrm{a}_{1}\left(\left(\tilde{\mathbf{u}}, \theta_{1}, \theta_{2}, \omega_{1}, \omega_{2}\right),\left(\tilde{\mathbf{u}}, \theta_{1}, \theta_{2}, \omega_{1}, \omega_{2}\right)\right)+\max \left(\frac{\nu}{2}, c\right)\left\|\left(\tilde{\mathbf{u}}, \theta_{1}, \theta_{2}, \omega_{1}, \omega_{2}\right)\right\|_{\mathbb{H}}^{2} \geq c\left\|\left(\tilde{\mathbf{u}}, \theta_{1}, \theta_{2}, \omega_{1}, \omega_{2}\right)\right\|_{\mathbb{V}}^{2}
\end{aligned}
$$

Then we can easily conclude that $D\left(\left(-A_{1}\right)^{1 / 2}\right)=\mathbb{V}$. Moreover, according to $\left[5\right.$, Theorem 2.12, p. 115], $A_{1}$ generates an analytic semigroup on $\mathbb{H}$.

Now, we use the fact that $A-A_{1} \in \mathcal{L}(\mathbb{H})$, then according to [26, Corollary 2.2.], $A$ generates an analytic semigroup on $\mathbb{H}$.

A consequence of the previous result is that there exists $\lambda>0$ such that $\lambda I-A$ is positive. Moreover, $D(\lambda I-A)=D\left(A_{1}\right)$, then by interpolation, $D\left((\lambda I-A)^{1 / 2}\right)=D\left(\left(-A_{1}\right)^{1 / 2}\right)=\mathbb{V}$.

We are now in position to prove Proposition 2.2.

Proof of Proposition 2.2. Let us denote $\mathbf{F}=\Pi_{\mathbb{H}}\left(\mathbf{f}, 0,0, \mathcal{M}_{0,0}^{-1} \mathbf{s}\right)$ and $\mathbf{z}_{0}=\left(\mathbf{u}_{0}, 0,0, \omega_{1,0}, \omega_{2,0}\right)$. We have $\mathbf{F} \in$ $L^{2}(0, T ; H)$ and, according to the compatibility condtions $(1.41)$ (with $\left.\mathbf{u}^{i}=0\right), \mathbf{z}_{0} \in D\left(A^{1 / 2}\right)=\mathbb{V}$.

According to [5, Theorem 3.1, p. 143] and Lemma 2.8, the problem

$$
\left\{\begin{array}{l}
\mathbf{z}^{\prime}(t)=A \mathbf{z}(t)+\mathbf{F}(t), \quad t \geq 0, \\
\mathbf{z}(0)=\mathbf{z}_{0},
\end{array}\right.
$$

admits a unique solution $\mathbf{z} \in L^{2}(0, T ; D(A)) \cap H^{1}(0, T ; \mathbb{H})$ and there exists $C>0$ such that

$$
\|\mathbf{z}\|_{L^{2}(0, T ; D(A)) \cap H^{1}(0, T ; \mathbb{H})} \leq C\left(\|\mathbf{F}\|_{L^{2}(0, T ; H)}+\left\|\mathbf{z}_{0}\right\|_{\mathbb{V}}\right) .
$$

With the Sobolev embedding

$$
L^{2}(0, T ; D(A)) \cap H^{1}(0, T ; \mathbb{H}) \hookrightarrow \mathscr{C}^{0}([0, T] ; \mathbb{V}),
$$

we have

$$
\|\mathbf{z}\|_{L^{2}(0, T ; D(A)) \cap \mathscr{C}^{0}([0, T] ; \mathbb{V}) \cap H^{1}(0, T ; \mathbb{H})} \leq C\left(\|\mathbf{F}\|_{L^{2}(0, T ; \mathbb{H})}+\left\|\mathbf{z}_{0}\right\|_{\mathbb{V}}\right) .
$$

Moreover, $C$ can be taken independent from $T \in\left(0, T_{0}\right)$. To prove this statement, we consider

$$
\forall t \in\left[0, T_{0}\right], \quad \tilde{\mathbf{F}}(t)= \begin{cases}\mathbf{F}(t) & \text { if } t \in[0, T] \\ 0 & \text { if } \left.t \in] T, T_{0}\right]\end{cases}
$$

If $\tilde{\mathbf{z}}$ is the solution on $\left[0, T_{0}\right]$ of

$$
\left\{\begin{array}{l}
\tilde{\mathbf{z}}^{\prime}=A \tilde{\mathbf{z}}+\tilde{\mathbf{F}} \\
\tilde{\mathbf{z}}(0)=\mathbf{z}_{0},
\end{array}\right.
$$

then for $t \leq T, \tilde{\mathbf{z}}(t)=\mathbf{z}(t)$. We have the inequality

$$
\|\tilde{\mathbf{z}}\|_{L^{2}\left(0, T_{0} ; D(A)\right) \cap \mathscr{C}^{0}\left(\left[0, T_{0}\right] ; \mathbb{V}\right) \cap H^{1}\left(0, T_{0} ; \mathbb{H}\right)} \leq C\left(\|\tilde{\mathbf{F}}\|_{L^{2}\left(0, T_{0} ; \mathbb{H}\right)}+\left\|\mathbf{z}_{0}\right\|_{\mathbb{V}}\right),
$$

where $C$ does not depend on $T$. Moreover,

$$
\|\mathbf{z}\|_{L^{2}(0, T ; D(A)) \cap \mathscr{C}^{0}([0, T] ; \mathbb{V}) \cap H^{1}(0, T ; \mathbb{H})} \leq\|\tilde{\mathbf{z}}\|_{L^{2}\left(0, T_{0} ; D(A)\right) \cap \mathscr{C}^{0}\left(\left[0, T_{0}\right] ; \mathbb{V}\right) \cap H^{1}\left(0, T_{0} ; \mathbb{H}\right)},
$$

and

$$
\|\tilde{\mathbf{F}}\|_{L^{2}\left(0, T_{0} ; H\right)}=\|\mathbf{F}\|_{L^{2}(0, T ; \mathbb{H})} .
$$

By combining these arguments, we get (2.10) with $C$ independent from $T$. 
Now, if we write $\mathbf{z}=\left(\tilde{\mathbf{u}}, \theta_{1}, \theta_{2}, \omega_{1}, \omega_{2}\right)$, problem (2.8) becomes

$$
\frac{\mathrm{d}}{\mathrm{d} t}\left(\begin{array}{c}
\tilde{\mathbf{u}} \\
\theta_{1} \\
\theta_{2} \\
\omega_{1} \\
\omega_{2}
\end{array}\right)=\Pi_{\mathbb{H}}\left(\begin{array}{l}
\operatorname{div} \sigma_{F}(\tilde{\mathbf{u}}, p)+\mathbf{f} \\
\omega_{1} \\
\omega_{2} \\
\mathcal{M}_{0,0}^{-1}\left(\mathbf{s}+\left(\int_{\partial S_{0}}-\sigma_{F}(\tilde{\mathbf{u}}, p) \mathbf{n}_{0} \cdot \partial_{\theta_{j}} \mathbf{\Phi}(0,0, .) \mathrm{d} \gamma_{y}\right)_{j=1 . .2}\right)
\end{array}\right)
$$

where $p \in L^{2}\left(0, T ; H_{\beta}^{1}\left(\mathscr{F}_{0}\right)\right)$. Then, Lemma 2.3 implies that there exists $q \in L^{2}\left(0, T ; H^{1}\left(\mathscr{F}_{0}\right)\right)$ such that $\left(\tilde{\mathbf{u}}, p+q, \theta_{1}, \theta_{2}\right)$ satisfies the linear problem $(2.1)$. Moreover, according to $(2.10)$, we have $\left(\theta_{1}, \theta_{2}\right) \in H^{2}\left(0, T ; \mathbb{R}^{2}\right)$, $\tilde{\mathbf{u}} \in H^{1}\left(0, T ; \mathbf{L}^{2}\left(\mathscr{F}_{0}\right)\right) \cap \mathscr{C}^{0}\left([0, T] ; \mathbf{H}^{1}\left(\mathscr{F}_{0}\right)\right) \cap L^{2}\left(0, T ; \mathbf{H}_{\beta}^{2}\left(\mathscr{F}_{0}\right)\right), \tilde{p}=p+q \in L^{2}\left(0, T ; H_{\beta}^{1}\left(\mathscr{F}_{0}\right)\right)$ and

$$
\begin{aligned}
\|\tilde{\mathbf{u}}\|_{L^{2}\left(0, T ; \mathbf{H}_{\beta}^{2}\left(\mathscr{F}_{0}\right)\right)} & \cap \mathscr{C}^{0}\left([0, T] ; \mathbf{H}^{1}\left(\mathscr{F}_{0}\right)\right) \cap H^{1}\left(0, T ; \mathbf{L}^{2}\left(\mathscr{F}_{0}\right)\right) \\
& \leq C\left(\|\tilde{p}\|_{L^{2}\left(0, T ; H_{\beta}^{1}\left(\mathscr{F}_{0}\right)\right)}+\left\|\left(\theta_{1}, \theta_{2}\right)\right\|_{\Theta_{T}}\right. \\
& \left.\leq C\left\|_{\mathbf{H}^{1}\left(\mathscr{F}_{0}\right)}+\left|\omega_{1,0}\right|+\left|\omega_{2,0}\right|+\right\| \mathbf{f}\left\|_{L^{2}\left(0, T ; \mathbf{L}^{2}\left(\mathscr{F}_{0}\right)\right)}+\right\| \mathbf{s} \|_{L^{2}\left(0, T ; \mathbb{R}^{2}\right)}\right)
\end{aligned}
$$

This concludes the proof of Proposition 2.2.

\subsection{Linearized problem with nonhomogeneous boundary data}

Let us now consider two more nonhomogeneous data: one datum $\mathbf{g}$ on the boundary of the structure $\partial S_{0}$ and one datum $\mathbf{u}^{i}$ on the inflow boundary region $\Gamma_{\mathrm{i}}$. Let $T_{0}>0$, we study

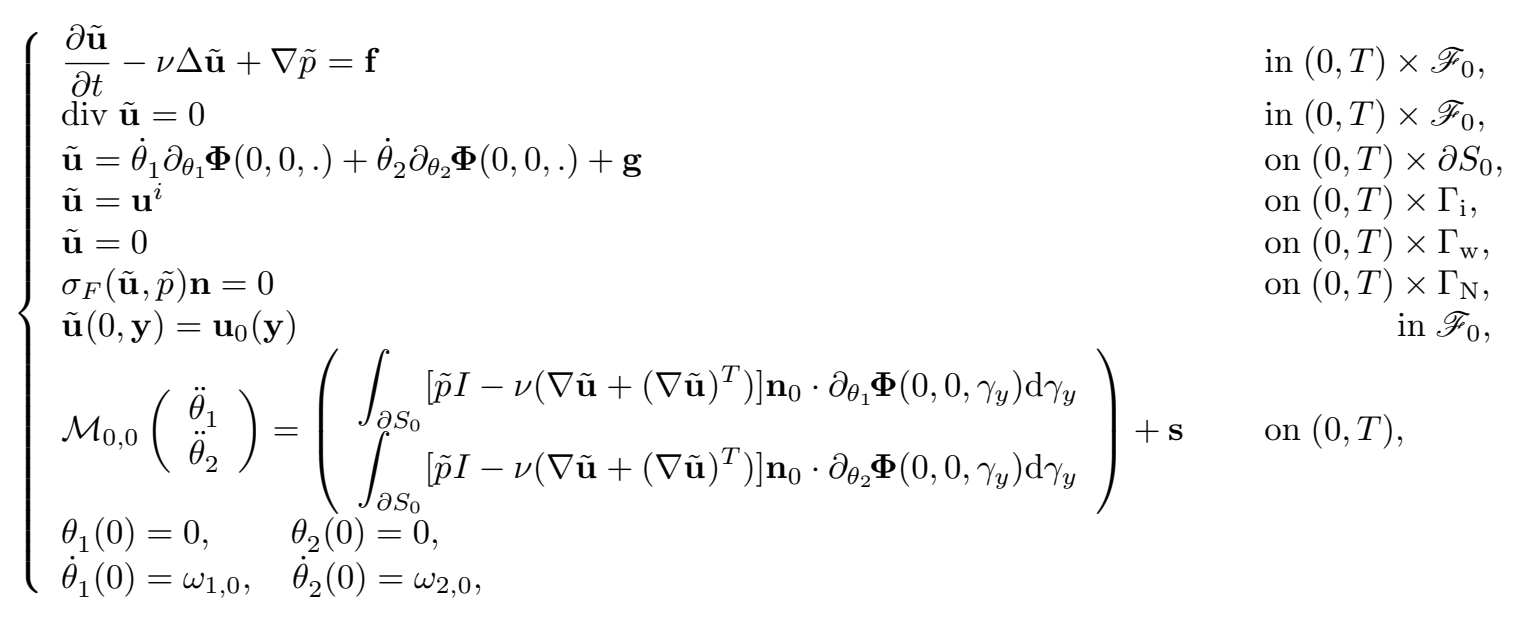

where the source terms and data are $\mathbf{f} \in L^{2}\left(0, T ; \mathbf{L}^{2}\left(\mathscr{F}_{0}\right)\right), \mathbf{g} \in H^{1}\left(0, T ; \mathbf{H}^{3 / 2}\left(\partial S_{0}\right)\right), \mathbf{u}^{i} \in H^{1}\left(0, T_{0} ; \mathbf{U}^{i}\right)$ and $\mathbf{s} \in L^{2}\left(0, T ; \mathbb{R}^{2}\right)$.

We prove in this section the following result.

Proposition 2.9. There exists a constant $C>0$ such that for all $T \in\left(0, T_{0}\right), C$ does not depend on $T$, for all $\mathbf{u}^{i} \in H^{1}\left(0, T_{0} ; \mathbf{U}^{i}\right), \mathbf{u}_{0} \in \mathbf{H}^{1}\left(\mathscr{F}_{0}\right)$ and $\left(\omega_{1,0}, \omega_{2,0}\right) \in \mathbb{R}^{2}$ satisfying the compatibility conditions $(1.41)$ and every $(\mathbf{f}, \mathbf{g}, \mathbf{s}) \in \mathbb{F}_{T} \times \mathbb{G}_{T} \times \mathbb{S}_{T}$ with $\mathbf{g}(0)=0$, problem (2.11) admits a unique solution

$$
\left(\tilde{\mathbf{u}}, \tilde{p}, \theta_{1}, \theta_{2}\right) \in \mathbb{U}_{T} \times \mathbb{P}_{T} \times \Theta_{T}
$$

with

$$
\begin{aligned}
\|\tilde{\mathbf{u}}\|_{\mathbb{U}_{T}}+\|\tilde{p}\|_{\mathbb{P}_{T}}+\left\|\left(\theta_{1}, \theta_{2}\right)\right\|_{\Theta_{T}} \leq C\left(\left\|\mathbf{u}_{0}\right\|_{\mathbf{H}^{1}\left(\mathscr{F}_{0}\right)}\right. & +\left|\omega_{1,0}\right|+\left|\omega_{2,0}\right| \\
& \left.+\|\mathbf{f}\|_{\mathbb{F}_{T}}+\|\mathbf{g}\|_{\mathbb{G}_{T}}+\|\mathbf{s}\|_{S_{T}}+\left\|\mathbf{u}^{i}\right\|_{H^{1}\left(0, T_{0} ; \mathbf{U}^{i}\right)}\right) .
\end{aligned}
$$

Proposition 2.9 is proven at the end of the section. The proof uses the following lifting result for the terms $\mathrm{g}$ and $\mathbf{u}^{i}$.

Lemma 2.10. For every $\mathbf{g} \in \mathbf{H}^{3 / 2}\left(\partial S_{0}\right)$ and every $\mathbf{u}^{i} \in \mathbf{U}^{i}$, there exists $\overline{\mathbf{u}} \in \mathbf{H}^{2}\left(\mathscr{F}_{0}\right)$ satisfying

$$
\begin{cases}\operatorname{div} \overline{\mathbf{u}}=0 & \text { in } \mathscr{F}_{0} \\ \overline{\mathbf{u}}=\mathbf{g} & \text { on } \partial S_{0} \\ \overline{\mathbf{u}}=\mathbf{u}^{i} & \text { on } \Gamma_{\mathrm{i}} \\ \overline{\mathbf{u}}=0 & \text { on } \Gamma_{\mathrm{w}} \\ \left(\nabla \overline{\mathbf{u}}+(\nabla \overline{\mathbf{u}})^{T}\right) \mathbf{n}=0 & \text { on } \Gamma_{\mathrm{N}}\end{cases}
$$

with

$$
\|\overline{\mathbf{u}}\|_{\mathbf{H}^{2}\left(\mathscr{F}_{0}\right)} \leq C\left(\left\|\mathbf{u}^{i}\right\|_{\mathbf{U}^{i}}+\|\mathbf{g}\|_{\mathbf{H}^{3 / 2}\left(\partial S_{0}\right)}\right)
$$


Note that despite the presence of corners, we recover the expected regularity of the lifting for smooth domains.

Remark 2.11. For the sake of readability, from this point onwards all terms $\mathrm{d} \mathbf{y}$ and $\mathrm{d} \gamma_{y}$ are omitted in the integrals.

Proof of Lemma 2.10. The lifting result has been established for $\mathbf{u}^{i}=0$ on the inflow region in [25, Theorem 2.16]. We first lift the inflow boundary condition $\mathbf{u}^{i} \neq 0$ in $\Omega$ and then we use the aforementioned result.

Lifting of the inflow boundary condition. Let us look for a function $\mathbf{v}$ defined on the entire domain $\Omega$ and satisfying

$$
\begin{cases}\operatorname{div} \mathbf{v}=0 & \text { in } \Omega \\ \mathbf{v}=\mathbf{u}^{i} & \text { on } \Gamma_{\mathrm{i}} \\ \mathbf{v}=0 & \text { on } \Gamma_{\mathrm{w}} \\ \left(\nabla \mathbf{v}+(\nabla \mathbf{v})^{T}\right) \mathbf{n}=0 & \text { on } \Gamma_{\mathrm{N}}\end{cases}
$$

As $\mathbf{v}$ is divergence-free and $\Omega$ is simply connected, we look for it under the form $\mathbf{v}=\nabla^{\perp} \psi$, where $\psi$ is a scalar-valued function. In the geometry considered, $\Gamma_{\mathrm{i}}, \Gamma_{\mathrm{t}}, \Gamma_{\mathrm{b}}$ and $\Gamma_{\mathrm{N}}$ are straight lines, hence $\partial_{\mathbf{n}}$ is written as $\pm \partial_{y_{1}}$ or $\pm \partial_{y_{2}}$ according on the considered part of the boundary.

We can prove that $\psi$ has to satisfy the conditions

$$
\begin{array}{lll}
\partial_{y_{2}} \psi=-u_{1}^{i} & \text { and } \partial_{y_{1}} \psi=u_{2}^{i} & \text { on } \Gamma_{\mathrm{i}}, \\
\partial_{y_{1}} \psi=0 & \text { and } \partial_{y_{2}} \psi=0 & \text { on } \Gamma_{\mathrm{b}}, \\
\partial_{y_{1}} \psi=0 & \text { and } \partial_{y_{2}} \psi=0 & \text { on } \Gamma_{\mathrm{t}} \\
\partial_{y_{1}} \partial_{y_{2}} \psi=0 & \text { and } \partial_{y_{1}}^{2} \psi-\partial_{y_{2}}^{2} \psi=0 & \text { on } \Gamma_{\mathrm{N}}
\end{array}
$$

We choose to meet these conditions in the following way:

$$
\begin{aligned}
& \psi\left(y_{2}\right)=-\int_{0}^{y_{2}} u_{1}^{i} \quad \text { and } \partial_{y_{1}} \psi=u_{2}^{i} \quad \text { on } \Gamma_{\mathrm{i}}, \\
& \psi=0 \quad \text { and } \partial_{y_{2}} \psi=0 \quad \text { on } \Gamma_{\mathrm{b}} \text {, } \\
& \psi=-\int_{\Gamma_{\mathrm{i}}} u_{1}^{i} \quad \text { and } \partial_{y_{2}} \psi=0 \quad \text { on } \Gamma_{\mathrm{t}} . \\
& \psi\left(y_{2}\right)=-\eta\left(y_{2}\right) \int_{\Gamma_{\mathrm{i}}} u_{1}^{i}, \quad \partial_{y_{1}} \psi=0 \quad \text { and } \partial_{y_{1}}^{2} \psi=-\mathrm{d}_{y_{2}}^{2} \eta\left(y_{2}\right) \int_{\Gamma_{\mathrm{i}}} u_{1}^{i} \quad \text { on } \Gamma_{\mathrm{N}},
\end{aligned}
$$

where $\eta$ is a $\mathscr{C}^{\infty}$ function on $[0,1]$ satisfying

$$
\forall y_{2} \in[0,1], \quad \eta\left(y_{2}\right)=\left\{\begin{array}{lll}
0 & \text { if } & y_{2} \in[0,1 / 4], \\
\in[0,1] & \text { if } & \left.y_{2} \in\right] 1 / 4,3 / 4[, \\
1 & \text { if } & y_{2} \in[3 / 4,1] .
\end{array}\right.
$$

The theorem [16, Theorem 1.6.1.5, p.69] with $m=3$ and $d=2$ gives the existence of $\psi \in H^{3}(\Omega)$ fulfilling (2.16) under the compatibility conditions:

$$
\text { there exist } \alpha_{1} \text { and } \alpha_{2}>0 \text { such that }\left\{\begin{array}{l}
\int_{0}^{\alpha_{1}} \frac{\left|\partial_{y_{2}} u_{2}^{i}\right|^{2}}{y_{2}}<+\infty \\
\int_{1-\alpha_{2}}^{1} \frac{\left|\partial_{y_{2}} u_{2}^{i}\right|^{2}}{1-y_{2}}<+\infty
\end{array}\right.
$$

These conditions are the ones in the definition of $\mathbf{U}^{i}$ in (1.40) with $\alpha_{1}=\alpha_{2}=1 / 4$. Moreover we have the estimate

$$
\|\mathbf{v}\|_{\mathbf{H}^{2}(\Omega)} \leq c\|\psi\|_{H^{3}(\Omega)} \leq C\left\|\mathbf{u}^{i}\right\|_{\mathbf{H}^{3 / 2}\left(\Gamma_{\mathrm{i}}\right)} .
$$

The divergence-free field $\mathbf{v}=\nabla^{\perp} \psi \in \mathbf{H}^{2}(\Omega)$ satisfies (2.15).

Lifting of the structure velocity. Now, $\tilde{\mathbf{v}}=\overline{\mathbf{u}}-\mathbf{v}_{\mid \mathscr{F}_{0}}$ has to satisfy

$$
\begin{cases}\operatorname{div} \tilde{\mathbf{v}}=0 & \text { in } \mathscr{F}_{0} \\ \tilde{\mathbf{v}}=\mathbf{g}-\mathbf{v} & \text { on } \partial S_{0} \\ \tilde{\mathbf{v}}=0 & \text { on } \Gamma_{\mathrm{i}} \\ \tilde{\mathbf{v}}=0 & \text { on } \Gamma_{\mathrm{w}} \\ \left(\nabla \tilde{\mathbf{v}}+(\nabla \tilde{\mathbf{v}})^{T}\right) \mathbf{n}=0 & \text { on } \Gamma_{\mathrm{N}}\end{cases}
$$

According to [25, Theorem 2.16], such $\tilde{\mathbf{v}}$ exists in $\mathbf{H}^{2}\left(\mathscr{F}_{0}\right)$ as soon as $\mathbf{g}-\mathbf{v} \in \mathbf{H}^{3 / 2}\left(\partial S_{0}\right)$. Moreover, we have the estimate

$$
\|\tilde{\mathbf{v}}\|_{\mathbf{H}^{2}\left(\mathscr{F}_{0}\right)} \leq C\|\mathbf{g}-\mathbf{v}\|_{\mathbf{H}^{3 / 2}\left(\partial S_{0}\right)} \leq C\left(\|\mathbf{g}\|_{\mathbf{H}^{3 / 2}\left(\partial S_{0}\right)}+\|\mathbf{v}\|_{\mathbf{H}^{2}(\Omega)}\right) .
$$

This yields the expected result since $\overline{\mathbf{u}}=\tilde{\mathbf{v}}+\mathbf{v}_{\mid \mathscr{F}_{0}}$, the estimate (2.14) comes from (2.19) and (2.20). 
We can now prove Proposition 2.9 in the following way.

Proof of Proposition 2.9. Let $\mathbf{u}^{i} \in H^{1}\left(0, T_{0} ; \mathbf{U}^{i}\right), \mathbf{u}_{0} \in \mathbf{H}^{1}\left(\mathscr{F}_{0}\right)$ and $\left(\omega_{1,0}, \omega_{2,0}\right) \in \mathbb{R}^{2}$ satisfying the compatibility conditions (1.41). Let $(\mathbf{f}, \mathbf{g}, \mathbf{s}) \in \mathbb{F}_{T} \times \mathbb{G}_{T} \times \mathbb{S}_{T}$ with $\mathbf{g}(0)=0$.

Let $\overline{\mathbf{u}} \in H^{1}\left(0, T ; \mathbf{H}^{2}\left(\mathscr{F}_{0}\right)\right)$ be such that $\overline{\mathbf{u}}(t)$ is the solution to $(2.13)$, it fulfils

$$
\|\overline{\mathbf{u}}\|_{H^{1}\left(0, T ; \mathbf{H}^{2}\left(\mathscr{F}_{0}\right)\right)} \leq C\left(\left\|\mathbf{u}^{i}\right\|_{H^{1}\left(0, T_{0} ; \mathbf{U}^{i}\right)}+\|\mathbf{g}\|_{H^{1}\left(0, T ; \mathbf{H}^{3 / 2}\left(\partial S_{0}\right)\right)}\right) .
$$

The lifting $\overline{\mathbf{u}}$ also belongs to $\mathscr{C}^{0}\left([0, T] ; \mathbf{H}^{2}\left(\mathscr{F}_{0}\right)\right)$, and as $\mathbf{g}(0)=0$, we have

$$
\|\overline{\mathbf{u}}\|_{\mathscr{C}^{0}\left([0, T] ; \mathbf{H}^{2}\left(\mathscr{F}_{0}\right)\right)} \leq C\left(\left\|\mathbf{u}^{i}\right\|_{H^{1}\left(0, T_{0} ; \mathbf{U}^{i}\right)}+\|\mathbf{g}\|_{H^{1}\left(0, T ; \mathbf{H}^{3 / 2}\left(\partial S_{0}\right)\right)}\right),
$$

where $C$ does not depend on $T$.

Let $\left(\hat{\mathbf{u}}, \tilde{p}, \theta_{1}, \theta_{2}\right)$ be the solution to

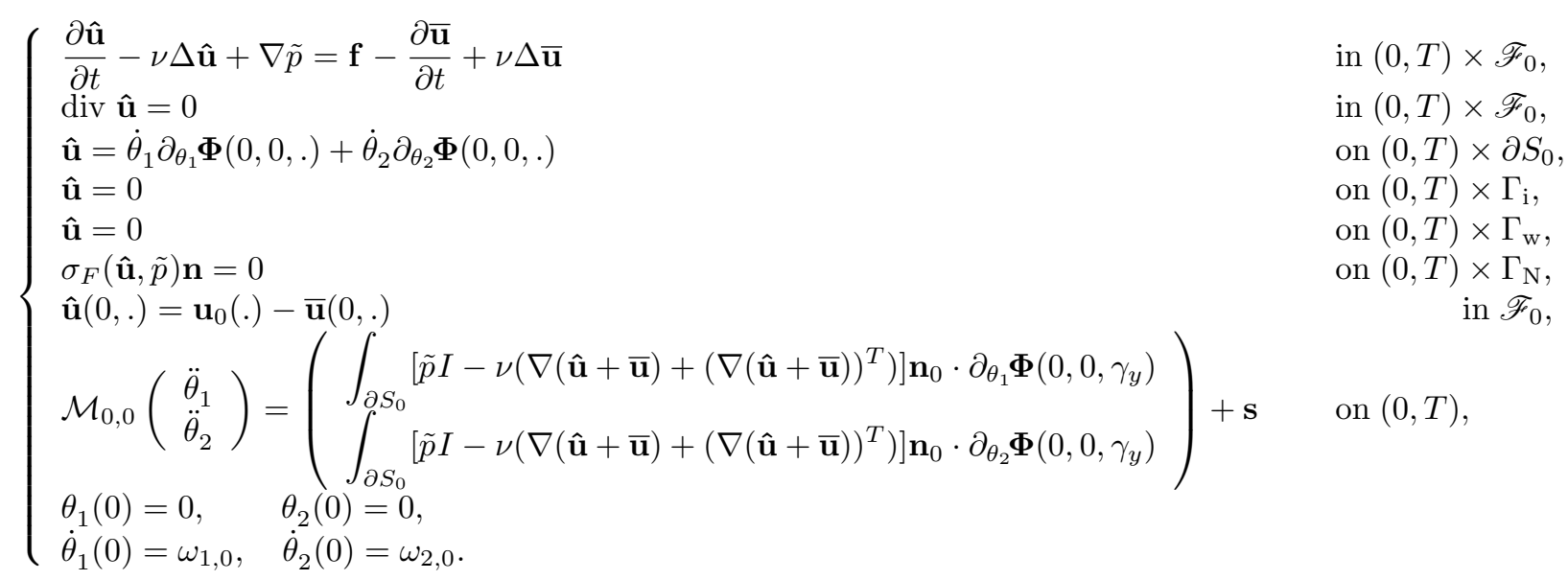

We have

$$
\begin{aligned}
& \mathbf{f}-\frac{\partial \overline{\mathbf{u}}}{\partial t}+\nu \Delta \overline{\mathbf{u}} \in L^{2}\left(0, T ; \mathbf{L}^{2}\left(\mathscr{F}_{0}\right)\right) \\
& \mathbf{u}_{0}(.)-\overline{\mathbf{u}}(0, .)=0 \text { on } \Gamma_{\mathrm{i}} \\
& s_{j}+\int_{\partial S_{0}}-\nu\left(\nabla \hat{\mathbf{u}}+(\nabla \hat{\mathbf{u}})^{T}\right) \mathbf{n}_{0} \cdot \partial_{\theta_{j}} \mathbf{\Phi}\left(0,0, \gamma_{y}\right) \in L^{2}(0, T) .
\end{aligned}
$$

Then, according to Proposition 2.2, ( $\left.\hat{\mathbf{u}}, \tilde{p}, \theta_{1}, \theta_{2}\right) \in \mathbb{U}_{T} \times \mathbb{P}_{T} \times \Theta_{T}$ and we have (2.2) with $\hat{\mathbf{u}}$ instead of $\tilde{\mathbf{u}}$.

Now, we consider $\tilde{\mathbf{u}}=\hat{\mathbf{u}}+\overline{\mathbf{u}}$, then $\left(\tilde{\mathbf{u}}, \tilde{p}, \theta_{1}, \theta_{2}\right) \in \mathbb{U}_{T} \times \mathbb{P}_{T} \times \Theta_{T}$ and (2.12) is a consequence of $(2.21)-(2.22)$ and (2.2).

Remark 2.12. Note that a larger space than $H^{1}\left(0, T_{0} ; \mathbf{U}^{i}\right)$ could be considered for $\mathbf{u}^{i}$. Indeed, we use a lifting in space only, inducing the requirement $\mathbf{u}^{i} \in H^{1}\left(0, T_{0} ; \mathbf{U}^{i}\right)$. Using a space-time lifting would be slightly more complicated (see [27]), but would allow a larger space for the inflow boundary datum $\mathbf{u}^{i}$.

\section{Local existence of solution to the full problem}

In this section, we study the nonlinear problem. We recall that $\theta_{1,0}=\theta_{2,0}=0$. At first, we rewrite the equations (1.24) in the fixed domain $\mathscr{F}_{0}$, then we prove existence of a solution to this problem.

\subsection{The equations in a fixed domain}

Our goal is to write the equations (1.24) in the fixed domain $\mathscr{F}_{0}$. To do so, we use the diffeomorphism defined in (1.37). We denote $\mathcal{J}_{\boldsymbol{\Phi}}$ its Jacobian matrix and $\operatorname{cof}\left(\mathcal{J}_{\boldsymbol{\Phi}}\right)$ the cofactor matrix of $\mathcal{J}_{\boldsymbol{\Phi}}$. We use the change of variables

$$
\forall t \in[0, T], \quad \forall \mathbf{y} \in \mathscr{F}_{0}, \quad\left\{\begin{array}{l}
\tilde{\mathbf{u}}(t, \mathbf{y})=\operatorname{cof}\left(\mathcal{J}_{\mathbf{\Phi}}\left(\theta_{1}(t), \theta_{2}(t), \mathbf{y}\right)\right)^{T} \mathbf{u}\left(t, \mathbf{\Phi}\left(\theta_{1}(t), \theta_{2}(t), \mathbf{y}\right)\right) \\
\tilde{p}(t, \mathbf{y})=p\left(t, \boldsymbol{\Phi}\left(\theta_{1}(t), \theta_{2}(t), \mathbf{y}\right)\right) .
\end{array}\right.
$$

This choice is motivated by the fact that, according to [10, Lemma 3.1], we get div $\tilde{\mathbf{u}}=0$.

In the sequel, $v_{i}$ denotes the $i^{t h}$ component of the vector $\mathbf{v}$. We recall that $\boldsymbol{\Psi}\left(\theta_{1}, \theta_{2},.\right)$ is the inverse diffeomorphism of $\boldsymbol{\Phi}\left(\theta_{1}, \theta_{2},.\right)$. To compute the equations satisfied by $\left(\tilde{\mathbf{u}}, \tilde{p}, \theta_{1}, \theta_{2}\right)$, we use the following explicit formula:

$$
\mathbf{u}(t, \mathbf{x})=\operatorname{cof}\left(\mathcal{J}_{\Psi}\left(\theta_{1}(t), \theta_{2}(t), \mathbf{x}\right)\right)^{T} \tilde{\mathbf{u}}\left(t, \mathbf{\Psi}\left(\theta_{1}(t), \theta_{2}(t), \mathbf{x}\right)\right)
$$


we have

$$
\begin{aligned}
\partial_{t} \mathbf{u}(t, \mathbf{x})= & \operatorname{cof}\left(\frac{\mathrm{d}}{\mathrm{d} t} \mathcal{J}_{\Psi}\left(\theta_{1}(t), \theta_{2}(t), \mathbf{x}\right)\right)^{T} \tilde{\mathbf{u}}\left(t, \Psi\left(\theta_{1}(t), \theta_{2}(t), \mathbf{x}\right)\right) \\
& +\operatorname{cof}\left(\mathcal{J}_{\Psi}\left(\theta_{1}(t), \theta_{2}(t), \mathbf{x}\right)\right)^{T} \partial_{t} \tilde{\mathbf{u}}\left(t, \Psi\left(\theta_{1}(t), \theta_{2}(t), \mathbf{x}\right)\right) \\
& +\operatorname{cof}\left(\mathcal{J}_{\Psi}\left(\theta_{1}(t), \theta_{2}(t), \mathbf{x}\right)\right)^{T} \nabla_{\mathbf{y}} \tilde{\mathbf{u}}\left(t, \Psi\left(\theta_{1}(t), \theta_{2}(t), \mathbf{x}\right)\right) \frac{\mathrm{d}}{\mathrm{d} t} \boldsymbol{\Psi}\left(\theta_{1}(t), \theta_{2}(t), \mathbf{x}\right), \\
\partial_{x_{j}} \mathbf{u}(t, \mathbf{x})= & \operatorname{cof}\left(\partial_{x_{j}} \mathcal{J}_{\Psi}\left(\theta_{1}(t), \theta_{2}(t), \mathbf{x}\right)\right)^{T} \tilde{\mathbf{u}}\left(t, \Psi\left(\theta_{1}(t), \theta_{2}(t), \mathbf{x}\right)\right) \\
& +\operatorname{cof}\left(\mathcal{J}_{\Psi}\left(\theta_{1}(t), \theta_{2}(t), \mathbf{x}\right)\right)^{T} \nabla_{\mathbf{y}} \tilde{\mathbf{u}}\left(t, \Psi\left(\theta_{1}(t), \theta_{2}(t), \mathbf{x}\right)\right) \partial_{x_{j}} \Psi\left(\theta_{1}(t), \theta_{2}(t), \mathbf{x}\right),
\end{aligned}
$$

and

$$
\begin{aligned}
\partial_{x_{j}}^{2} \mathbf{u}(t, \mathbf{x})= & \operatorname{cof}\left(\partial_{x_{j}}^{2} \mathcal{J}_{\mathbf{\Psi}}\left(\theta_{1}(t), \theta_{2}(t), \mathbf{x}\right)\right)^{T} \tilde{\mathbf{u}}\left(t, \mathbf{\Psi}\left(\theta_{1}(t), \theta_{2}(t), \mathbf{x}\right)\right) \\
& +2 \operatorname{cof}\left(\partial_{x_{j}} \mathcal{J}_{\Psi}\left(\theta_{1}(t), \theta_{2}(t), \mathbf{x}\right)\right)^{T} \nabla_{\mathbf{y}} \tilde{\mathbf{u}}\left(t, \mathbf{\Psi}\left(\theta_{1}(t), \theta_{2}(t), \mathbf{x}\right)\right) \partial_{x_{j}} \Psi\left(\theta_{1}(t), \theta_{2}(t), \mathbf{x}\right) \\
& +\operatorname{cof}\left(\mathcal{J}_{\Psi}\left(\theta_{1}(t), \theta_{2}(t), \mathbf{x}\right)\right)^{T} \sum_{k} \partial_{y_{k}} \nabla_{\mathbf{y}} \tilde{\mathbf{u}}\left(t, \Psi\left(\theta_{1}(t), \theta_{2}(t), \mathbf{x}\right)\right) \partial_{x_{j}} \mathbf{\Psi}\left(\theta_{1}(t), \theta_{2}(t), \mathbf{x}\right) \partial_{x_{j}} \Psi_{k}\left(\theta_{1}(t), \theta_{2}(t), \mathbf{x}\right) \\
& +\operatorname{cof}\left(\mathcal{J}_{\Psi}\left(\theta_{1}(t), \theta_{2}(t), \mathbf{x}\right)\right)^{T} \nabla_{\mathbf{y}} \tilde{\mathbf{u}}\left(t, \Psi\left(\theta_{1}(t), \theta_{2}(t), \mathbf{x}\right)\right) \partial_{x_{j}}^{2} \mathbf{\Psi}\left(\theta_{1}(t), \theta_{2}(t), \mathbf{x}\right)
\end{aligned}
$$

Problem (1.24) in the fixed domain reads (2.11) where $\mathbf{f}, \mathbf{g}, \mathbf{s}$ are defined by

$$
\left\{\begin{array}{l}
\mathbf{f}=\mathbf{F}\left(\theta_{1}, \theta_{2}, \tilde{\mathbf{u}}, \tilde{p}\right)+\mathbf{f}_{\mathscr{F}}\left(t, \mathbf{\Phi}\left(\theta_{1}(t), \theta_{2}(t), \mathbf{y}\right)\right) \\
\mathbf{g}=\mathbf{G}\left(\theta_{1}, \theta_{2}, \dot{\theta}_{1}, \dot{\theta}_{2}\right) \\
\mathbf{s}=\mathbf{S}\left(\theta_{1}, \theta_{2}, \tilde{\mathbf{u}}, \tilde{p}\right)+\mathbf{f}_{\mathbf{s}}
\end{array}\right.
$$

where $\mathbf{F}, \mathbf{G}$ and $\mathbf{S}$ are nonlinear terms. We use the decomposition $\mathbf{F}\left(\theta_{1}, \theta_{2}, \tilde{\mathbf{u}}, \tilde{p}\right)=\mathbf{F}^{1}+\mathbf{F}^{2}+\mathbf{F}^{3}+\mathbf{F}^{4}+\mathbf{F}^{5}$. We write $\mathbf{\Phi}\left(\theta_{1}, \theta_{2},.\right)$ under the simpler notation $\boldsymbol{\Phi}$. The nonlinear terms are given as follows:

$$
\begin{aligned}
& \mathbf{F}^{1}\left(\theta_{1}, \theta_{2}, \tilde{\mathbf{u}}\right)=\left(I-\operatorname{cof}\left(\mathcal{J}_{\Psi}\left(\theta_{1}, \theta_{2}, \boldsymbol{\Phi}\right)\right)^{T}\right) \frac{\partial \tilde{\mathbf{u}}}{\partial t}, \\
& \mathbf{F}^{2}\left(\theta_{1}, \theta_{2}, \tilde{\mathbf{u}}\right)=-\operatorname{cof}\left(\left(\partial_{t} \mathcal{J}_{\Psi}\left(\theta_{1}, \theta_{2}, .\right)\right) \circ \boldsymbol{\Phi}\right)^{T} \tilde{\mathbf{u}}(t, \mathbf{y})-\operatorname{cof}\left(\mathcal{J}_{\Psi}\left(\theta_{1}, \theta_{2}, \boldsymbol{\Phi}\right)\right)^{T}\left(\nabla_{\mathbf{y}} \tilde{\mathbf{u}}\right)\left(\left(\partial_{t} \Psi\left(\theta_{1}, \theta_{2}, .\right)\right) \circ \boldsymbol{\Phi}\right), \\
& \mathbf{F}^{3}\left(\theta_{1}, \theta_{2}, \tilde{\mathbf{u}}\right)_{i}=\nu \sum_{j, k, \ell, m} \operatorname{cof}\left(\mathcal{J}_{\boldsymbol{\Psi}}\left(\theta_{1}, \theta_{2}, \boldsymbol{\Phi}\right)\right)_{k i} \frac{\partial^{2} \tilde{u}_{k}}{\partial y_{\ell} \partial y_{m}} \frac{\partial \Psi_{\ell}}{\partial x_{j}}\left(\theta_{1}, \theta_{2}, \boldsymbol{\Phi}\right) \frac{\partial \Psi_{m}}{\partial x_{j}}\left(\theta_{1}, \theta_{2}, \boldsymbol{\Phi}\right) \\
& +2 \nu \sum_{j, k, \ell} \operatorname{cof}\left(\partial_{x_{j}} \mathcal{J}_{\Psi}\left(\theta_{1}, \theta_{2}, \boldsymbol{\Phi}\right)\right)_{k i} \frac{\partial \tilde{u}_{k}}{\partial y_{\ell}} \frac{\partial \Psi_{\ell}}{\partial x_{j}}\left(\theta_{1}, \theta_{2}, \boldsymbol{\Phi}\right) \\
& +\nu \sum_{j, k, \ell} \operatorname{cof}\left(\mathcal{J}_{\boldsymbol{\Psi}}\left(\theta_{1}, \theta_{2}, \boldsymbol{\Phi}\right)\right)_{k i} \frac{\partial \tilde{u}_{k}}{\partial y_{\ell}} \frac{\partial^{2} \Psi_{\ell}}{\partial x_{j}^{2}}\left(\theta_{1}, \theta_{2}, \boldsymbol{\Phi}\right) \\
& +\nu \sum_{j, k} \operatorname{cof}\left(\partial_{x_{j}}^{2} \mathcal{J}_{\mathbf{\Psi}}\left(\theta_{1}, \theta_{2}, \boldsymbol{\Phi}\right)\right)_{k i} \tilde{u}_{k}-\nu \Delta_{\mathbf{y}} \tilde{u}_{i}(t, \mathbf{y}) \\
& \mathbf{F}^{4}\left(\theta_{1}, \theta_{2}, \tilde{\mathbf{u}}\right)_{i}=-\sum_{j, k, r} \operatorname{cof}\left(\mathcal{J}_{\Psi}\left(\theta_{1}, \theta_{2}, \boldsymbol{\Phi}\right)\right)_{k j} \operatorname{cof}\left(\partial_{x_{j}} \mathcal{J}_{\Psi}\left(\theta_{1}, \theta_{2}\right)\right)_{r i} \tilde{u}_{k} \tilde{u}_{r} \\
& -\sum_{k, r} \operatorname{det}\left(\mathcal{J}_{\Psi}\left(\theta_{1}, \theta_{2}, \boldsymbol{\Phi}\right)\right)^{2} \frac{\partial \Phi_{i}}{\partial y_{r}} \frac{\partial \tilde{u}_{r}}{\partial y_{k}} \tilde{u}_{k} \\
& \mathbf{F}^{5}\left(\theta_{1}, \theta_{2}, \tilde{p}\right)=\left(I-\mathcal{J}_{\mathbf{\Psi}}\left(\theta_{1}, \theta_{2}, \mathbf{\Phi}\right)\right)^{T} \nabla_{\mathbf{y}} \tilde{p}, \\
& \mathbf{G}\left(\theta_{1}, \theta_{2}, \omega_{1}, \omega_{2}\right)=\sum_{j=1}^{2} \omega_{j}\left(\operatorname{cof}\left(\mathcal{J}_{\mathbf{\Phi}}\left(\theta_{1}, \theta_{2}, \mathbf{y}\right)\right)^{T} \partial_{\theta_{j}} \mathbf{\Phi}\left(\theta_{1}, \theta_{2}, \mathbf{y}\right)-\partial_{\theta_{j}} \mathbf{\Phi}(0,0, \mathbf{y})\right), \\
& \mathbf{S}\left(\theta_{1}, \theta_{2}, \tilde{\mathbf{u}}, \tilde{p}\right)=-\left(\mathcal{M}_{\theta_{1}, \theta_{2}}-\mathcal{M}_{0,0}\right)\left(\begin{array}{c}
\ddot{\theta}_{1} \\
\ddot{\theta}_{2}
\end{array}\right)+\mathbf{M}_{\mathbf{I}}\left(\theta_{1}, \theta_{2}, \dot{\theta}_{1}, \dot{\theta}_{2}\right) \\
& +\left(\begin{array}{l}
\int_{\partial S_{0}}\left|\mathcal{J}_{\boldsymbol{\Phi}} \mathbf{t}_{0}\right|\left[\tilde{p} I-\nu\left(\mathcal{G}\left(\theta_{1}, \theta_{2}, \tilde{\mathbf{u}}\right)+\mathcal{G}\left(\theta_{1}, \theta_{2}, \tilde{\mathbf{u}}\right)^{T}\right)\right] \mathbf{n}_{\theta_{1}, \theta_{2}}(\boldsymbol{\Phi}) \cdot \partial_{\theta_{1}} \mathbf{\Phi}\left(\theta_{1}, \theta_{2}, \gamma_{y}\right) \\
\int_{\partial S_{0}}\left|\mathcal{J}_{\boldsymbol{\Phi}} \mathbf{t}_{0}\right|\left[\tilde{p} I-\nu\left(\mathcal{G}\left(\theta_{1}, \theta_{2}, \tilde{\mathbf{u}}\right)+\mathcal{G}\left(\theta_{1}, \theta_{2}, \tilde{\mathbf{u}}\right)^{T}\right)\right] \mathbf{n}_{\theta_{1}, \theta_{2}}(\boldsymbol{\Phi}) \cdot \partial_{\theta_{2}} \mathbf{\Phi}\left(\theta_{1}, \theta_{2}, \gamma_{y}\right)
\end{array}\right) \\
& -\left(\begin{array}{l}
\int_{\partial S_{0}}^{S_{0}}\left[\tilde{p} I-\nu\left(\nabla \tilde{\mathbf{u}}+(\nabla \tilde{\mathbf{u}})^{T}\right)\right] \mathbf{n}_{0} \cdot \partial_{\theta_{1}} \boldsymbol{\Phi}\left(0,0, \gamma_{y}\right) \\
\int_{\partial S_{0}}\left[\tilde{p} I-\nu\left(\nabla \tilde{\mathbf{u}}+(\nabla \tilde{\mathbf{u}})^{T}\right)\right] \mathbf{n}_{0} \cdot \partial_{\theta_{2}} \mathbf{\Phi}\left(0,0, \gamma_{y}\right)
\end{array}\right)
\end{aligned}
$$

where $\mathbf{t}_{0}$ is a unit tangent vector to $\partial S_{0}, \mathbf{M}_{\mathbf{I}}$ and $\mathcal{M}_{\theta_{1}, \theta_{2}}$ are defined in (1.16), (1.17) and

$$
\left.\mathcal{G}\left(\theta_{1}, \theta_{2}, \tilde{\mathbf{u}}\right)_{i j}=\sum_{k} \operatorname{cof}\left[\partial_{x_{j}} \mathcal{J}_{\Psi}\left(\theta_{1}, \theta_{2}, .\right)\right) \circ \boldsymbol{\Phi}\right]_{k i} \tilde{u}_{k}+\sum_{k, \ell} \operatorname{cof}\left(\mathcal{J}_{\boldsymbol{\Psi}}\left(\theta_{1}, \theta_{2}, \boldsymbol{\Phi}\right)\right)_{k i} \frac{\partial \tilde{u}_{k}}{\partial y_{\ell}} \frac{\partial \Psi_{\ell}}{\partial x_{j}}\left(\theta_{1}, \theta_{2}, \boldsymbol{\Phi}\right) .
$$


For every $T>0$, we define the space

$$
\mathbb{N}_{T}=\left\{\left(\tilde{\mathbf{u}}, \tilde{p}, \theta_{1}, \theta_{2}\right) \in \mathbb{U}_{T} \times \mathbb{P}_{T} \times \Theta_{T} \text { with } \theta_{1}(0)=\theta_{2}(0)=0, \quad \text { and } \quad \forall t \in[0, T], \quad\left(\theta_{1}, \theta_{2}\right)(t) \in \mathbb{D}_{\Theta}\right\}
$$

that we endow with the norm

$$
\left\|\left(\tilde{\mathbf{u}}, \tilde{p}, \theta_{1}, \theta_{2}\right)\right\|_{\mathbb{N}_{T}}=\|\tilde{\mathbf{u}}\|_{\mathbb{U}_{T}}+\|\tilde{p}\|_{\mathbb{P}_{T}}+\left\|\left(\theta_{1}, \theta_{2}\right)\right\|_{\Theta_{T}} .
$$

We can state the following theorem.

Theorem 3.1. Let $T_{0}>0$. Let $\mathbf{u}^{i} \in H^{1}\left(0, T_{0} ; \mathbf{U}^{i}\right)$ and $\left(\mathbf{f}_{\mathscr{F}}, \mathbf{f}_{\mathbf{s}}\right) \in L^{2}\left(0, T_{0} ; \mathbf{W}^{1, \infty}(\Omega)\right) \times L^{2}\left(0, T_{0} ; \mathbb{R}^{2}\right)$. For every $\left(\mathbf{u}_{0}, \omega_{1,0}, \omega_{2,0}\right) \in \mathbf{H}^{1}\left(\mathscr{F}_{0}\right) \times \mathbb{R}^{2}$ satisfying the compatibility conditions $(1.41)$, there exists $T \in\left(0, T_{0}\right]$ such that problem (2.11) where the source terms are given by (3.1) admits a unique solution $\left(\tilde{\mathbf{u}}, \tilde{p}, \theta_{1}, \theta_{2}\right) \in \mathbb{N}_{T}$ satisfying the following estimate

$$
\left\|\tilde{\mathbf{u}}, \tilde{p}, \theta_{1}, \theta_{2}\right\|_{\mathbb{N}_{T}} \leq C\left(\left\|\mathbf{u}_{0}\right\|_{\mathbf{H}^{1}\left(\mathscr{F}_{0}\right)}+\left|\omega_{1,0}\right|+\left|\omega_{2,0}\right|+\left\|\mathbf{f}_{\mathscr{F}}\right\|_{L^{2}\left(0, T_{0} ; \mathbf{L}^{2}\left(\mathscr{F}_{0}\right)\right)}+\left\|\mathbf{u}^{i}\right\|_{H^{1}\left(0, T_{0} ; \mathbf{U}^{i}\right)}+\left\|\mathbf{f}_{\mathbf{s}}\right\|_{L^{2}\left(0, T_{0}\right)}\right),
$$

where $C$ does not depend on $T, \mathbf{f}_{\mathscr{F}}, \mathbf{f}_{\mathbf{s}}$ and $\mathbf{u}^{i}$.

This theorem is the rewriting of Theorem 1.8 in the fixed domain $\mathscr{F}_{0}$. To prove Theorem 3.1 , we use the results of Section 2 and a fixed point argument.

\subsection{Proof of Theorem 3.1}

Proof. We work in the fixed fluid domain $\mathscr{F}_{0}$. Let $T_{0}>0$.

Let $\mathbf{u}^{i} \in H^{1}\left(0, T_{0} ; \mathbf{U}^{i}\right)$ and $\left(\mathbf{u}_{0}, \omega_{1,0}, \omega_{2,0}\right) \in \mathbf{H}^{1}\left(\mathscr{F}_{0}\right) \times \mathbb{R}^{2}$ satisfying the compatibility conditions $(1.41)$.

We define an application $\Lambda^{T}$ on $\mathbb{N}_{T}$ such that for every $\left(\overline{\mathbf{u}}, \bar{p}, \bar{\theta}_{1}, \bar{\theta}_{2}\right) \in \mathbb{N}_{T},\left(\tilde{\mathbf{u}}, \tilde{p}, \theta_{1}, \theta_{2}\right)=\Lambda^{T}\left(\overline{\mathbf{u}}, \bar{p}, \bar{\theta}_{1}, \bar{\theta}_{2}\right) \in$ $\mathbb{U}_{T} \times \mathbb{P}_{T} \times \Theta_{T}$ is the solution to problem (2.11), where the nonhomogeneous terms are given by

$$
\begin{aligned}
& \mathbf{f}=\mathbf{F}\left(\bar{\theta}_{1}, \bar{\theta}_{2}, \overline{\mathbf{u}}, \bar{p}\right)+\mathbf{f}_{\mathscr{F}}\left(t, \mathbf{\Phi}\left(\bar{\theta}_{1}, \bar{\theta}_{2}, \mathbf{y}\right)\right), \\
& \mathbf{g}=\mathbf{G}\left(\bar{\theta}_{1}, \bar{\theta}_{2}, \dot{\bar{\theta}}_{1}, \dot{\bar{\theta}}_{2}\right), \\
& \mathbf{s}=\mathbf{S}\left(\bar{\theta}_{1}, \bar{\theta}_{2}, \overline{\mathbf{u}}, \bar{p}\right)+\mathbf{f}_{\mathbf{s}},
\end{aligned}
$$

where $\mathbf{F}, \mathbf{G}$ and $\mathbf{S}$ are given by (3.2). Note that $\Lambda^{T}$ is well defined, indeed if $\left(\overline{\mathbf{u}}, \bar{p}, \bar{\theta}_{1}, \bar{\theta}_{2}\right) \in \mathbb{N}_{T}$, we have $\mathbf{G}\left(\bar{\theta}_{1}, \bar{\theta}_{2}, \dot{\bar{\theta}}_{1}, \dot{\bar{\theta}}_{2}\right)(t=0)=0$ and we prove below that $(\mathbf{f}, \mathbf{g}, \mathbf{s})$ belongs to $\mathbb{F}_{T} \times \mathbb{G}_{T} \times \mathbb{S}_{T}$ (see Lemma 3.2 with $\left.\left(\tilde{\mathbf{u}}^{a}, \tilde{p}^{a}, \theta_{1}^{a}, \theta_{2}^{a}\right)=(0,0,0,0)\right)$. Then according to Proposition $2.9, \Lambda^{T}\left(\overline{\mathbf{u}}, \bar{p}, \bar{\theta}_{1}, \bar{\theta}_{2}\right)$ is uniquely defined. Note that $\Lambda^{T}$ depends on the initial data $\left(\mathbf{u}_{0}, \omega_{1,0}, \omega_{2,0}\right)$ and on the source term $\mathbf{u}^{i}$.

We take

$$
R=2 C\left(\left\|\mathbf{u}^{i}\right\|_{H^{1}\left(0, T_{0} ; \mathbf{U}^{i}\right)}+\left\|\mathbf{u}_{0}\right\|_{\mathbf{H}^{1}\left(\mathscr{F}_{0}\right)}+\left|\omega_{1,0}\right|+\left|\omega_{2,0}\right|+\left\|\mathbf{f}_{\mathscr{F}}\right\|_{L^{2}\left(0, T_{0} ; \mathbf{L}^{2}\left(\mathscr{F}_{0}\right)\right)}+\left\|\mathbf{f}_{\mathbf{S}}\right\|_{L^{2}\left(0, T_{0}\right)}\right),
$$

where $C$ is the constant of Proposition 2.9, so that Proposition 2.9 gives

$$
\left\|\Lambda^{T}(0,0,0,0)\right\|_{\mathbb{N}_{T}} \leq C\left(\left\|\mathbf{u}^{i}\right\|_{H^{1}\left(0, T_{0} ; \mathbf{U}^{i}\right)}+\left\|\mathbf{u}_{0}\right\|_{\mathbf{H}^{1}\left(\mathscr{F}_{0}\right)}+\left|\omega_{1,0}\right|+\left|\omega_{2,0}\right|+\left\|\mathbf{f}_{\mathscr{F}}\right\|_{L^{2}\left(0, T_{0} ; \mathbf{L}^{2}\left(\mathscr{F}_{0}\right)\right)}+\left\|\mathbf{f}_{\mathbf{s}}\right\|_{L^{2}\left(0, T_{0}\right)}\right)=R / 2 .
$$

The strategy adopted is based on the existence of $T>0$ such that $\Lambda^{T}$ is a contraction on

$$
\mathbb{B}_{R}(T)=\left\{\left(\tilde{\mathbf{u}}, \tilde{p}, \theta_{1}, \theta_{2}\right) \in \mathbb{N}_{T} \quad \text { with } \quad\left\|\left(\tilde{\mathbf{u}}, \tilde{p}, \theta_{1}, \theta_{2}\right)\right\|_{\mathbb{N}_{T}} \leq R\right\} .
$$

The domain $\mathbb{D}_{\Theta}$ is an open subset of $\mathbb{R}^{2}$ and $(0,0) \in \mathbb{D}_{\Theta}$, then there exists $r>0$ such that $B((0,0), r) \subset \mathbb{D}_{\Theta}$. Then for $T<r / R$, if $\left(., ., \theta_{1}, \theta_{2}\right) \in \mathbb{B}_{R}(T)$, we have $\left\|\dot{\theta}_{j}\right\|_{L^{\infty}(0, T)} \leq R$ and $\theta_{j}(0)=0$, then

$$
\left\|\theta_{j}\right\|_{L^{\infty}(0, T)} \leq T\left\|\dot{\theta}_{j}\right\|_{L^{\infty}(0, T)} \leq R T \leq r
$$

and we have for all $t \in(0, T),\left(\theta_{1}(t), \theta_{2}(t)\right) \in \mathbb{D}_{\Theta}$. In the sequel we choose $T_{0}>0$ such that $T_{0}<r / R$. Hence $\forall T \in[0, T], \Lambda^{T}: \mathbb{B}_{R}(T) \rightarrow \mathbb{N}_{T}$.

The solution to the nonlinear problem will be obtained as a fixed point of the application $\Lambda^{T}$ on $\mathbb{B}_{R}(T)$. We use the estimates of the following lemma.

Lemma 3.2. For every $R^{\prime}>0$, there exists a constant $C^{\prime}=C^{\prime}\left(R^{\prime}\right)>0$, such that for every $T \in\left(0, T_{0}\right)$, and every $\left(\tilde{\mathbf{u}}^{j}, \tilde{p}^{j}, \theta_{1}^{j}, \theta_{2}^{j}\right) \in \mathbb{B}_{R^{\prime}}(T)$, we have

$$
\begin{aligned}
\left\|\mathbf{F}\left(\theta_{1}^{a}, \theta_{2}^{a}, \tilde{\mathbf{u}}^{a}, \tilde{p}^{a}\right)-\mathbf{F}\left(\theta_{1}^{b}, \theta_{2}^{b}, \tilde{\mathbf{u}}^{b}, \tilde{p}^{b}\right)\right\|_{\mathbb{F}_{T}} & \leq C^{\prime} T^{1 / 4}\left(\left\|\tilde{\mathbf{u}}^{a}-\tilde{\mathbf{u}}^{b}\right\|_{\mathbb{U}_{T}}+\left\|\tilde{p}^{a}-\tilde{p}^{b}\right\|_{\mathbb{P}_{T}}+\left\|\theta^{a}-\theta^{b}\right\|_{\Theta_{T}}\right) \\
\left\|\mathbf{G}\left(\theta_{1}^{a}, \theta_{2}^{a}, \dot{\theta}_{1}^{a}, \dot{\theta}_{2}^{a}\right)-\mathbf{G}\left(\theta_{1}^{b}, \theta_{2}^{b}, \dot{\theta}_{1}^{b}, \dot{\theta}_{2}^{b}\right)\right\|_{\mathbb{G}_{T}} & \leq C^{\prime} T^{1 / 2}\left\|\theta^{a}-\theta^{b}\right\|_{\Theta_{T}} \\
\left\|\mathbf{S}\left(\theta_{1}^{a}, \theta_{2}^{a}, \tilde{\mathbf{u}}^{a}, \tilde{p}^{a}\right)-\mathbf{S}\left(\theta_{1}^{b}, \theta_{2}^{b}, \tilde{\mathbf{u}}^{b}, \tilde{p}^{b}\right)\right\|_{\mathbb{S}_{T}} & \leq C^{\prime} T^{1 / 2}\left(\left\|\tilde{\mathbf{u}}^{a}-\tilde{\mathbf{u}}^{b}\right\|_{\mathbb{U}_{T}}+\left\|\tilde{p}^{a}-\tilde{p}^{b}\right\|_{\mathbb{P}_{T}}+\left\|\theta^{a}-\theta^{b}\right\|_{\Theta_{T}}\right) \\
\left\|\mathbf{f}_{\mathscr{F}}\left(t, \mathbf{\Phi}\left(\theta_{1}^{a}, \theta_{2}^{a}, \mathbf{y}\right)\right)-\mathbf{f}_{\mathscr{F}}\left(t, \mathbf{\Phi}\left(\theta_{1}^{b}, \theta_{2}^{b}, \mathbf{y}\right)\right)\right\|_{\mathbb{F}_{T}} & \leq C^{\prime} T\left\|\theta^{a}-\theta^{b}\right\|_{\Theta_{T}}
\end{aligned}
$$


These estimates are proven in Appendix B.

For $\left(\tilde{\mathbf{u}}^{j}, \tilde{p}^{j}, \theta_{1}^{j}, \theta_{2}^{j}\right) \in \mathbb{B}_{R}(T)$, Proposition 2.9 yields the estimate

$$
\begin{aligned}
& \left\|\Lambda^{T}\left(\tilde{\mathbf{u}}^{a}, \tilde{p}^{a}, \theta_{1}^{a}, \theta_{2}^{a}\right)-\Lambda^{T}\left(\tilde{\mathbf{u}}^{b}, \tilde{p}^{b}, \theta_{1}^{b}, \theta_{2}^{b}\right)\right\|_{\mathbb{N}_{T}} \\
& \leq C\left(\left\|\mathbf{F}\left(\theta_{1}^{a}, \theta_{2}^{a}, \tilde{\mathbf{u}}^{a}, \tilde{p}^{a}\right)-\mathbf{F}\left(\theta_{1}^{b}, \theta_{2}^{b}, \tilde{\mathbf{u}}^{b}, \tilde{p}^{b}\right)\right\|_{\mathbb{F}_{T}}+\left\|\mathbf{G}\left(\theta_{1}^{a}, \theta_{2}^{a}, \dot{\theta}_{1}^{a}, \dot{\theta}_{2}^{a}\right)-\mathbf{G}\left(\theta_{1}^{b}, \theta_{2}^{b}, \dot{\theta}_{1}^{b}, \dot{\theta}_{2}^{b}\right)\right\|_{\mathbb{G}_{T}}\right. \\
& \left.\quad+\left\|\mathbf{S}\left(\theta_{1}^{a}, \theta_{2}^{a}, \tilde{\mathbf{u}}^{a}, \tilde{p}^{a}\right)-\mathbf{S}\left(\theta_{1}^{b}, \theta_{2}^{b}, \tilde{\mathbf{u}}^{b}, \tilde{p}^{b}\right)\right\|_{\mathbb{S}_{T}}+\left\|\mathbf{f}_{\mathscr{F}}\left(t, \boldsymbol{\Phi}\left(\theta_{1}^{a}, \theta_{2}^{a}, \mathbf{y}\right)\right)-\mathbf{f}_{\mathscr{F}}\left(t, \mathbf{\Phi}\left(\theta_{1}^{b}, \theta_{2}^{b}, \mathbf{y}\right)\right)\right\|_{\mathbb{F}_{T}}\right)
\end{aligned}
$$

and with Lemma 3.2, we have

$$
\left\|\Lambda^{T}\left(\tilde{\mathbf{u}}^{a}, \tilde{p}^{a}, \theta_{1}^{a}, \theta_{2}^{a}\right)-\Lambda^{T}\left(\tilde{\mathbf{u}}^{b}, \tilde{p}^{b}, \theta_{1}^{b}, \theta_{2}^{b}\right)\right\|_{\mathbb{N}_{T}} \leq K T^{1 / 4}\left(\left\|\tilde{\mathbf{u}}^{a}-\tilde{\mathbf{u}}^{b}\right\|_{\mathbb{U}_{T}}+\left\|\tilde{p}^{a}-\tilde{p}^{b}\right\|_{\mathbb{P}_{T}}+\left\|\theta^{a}-\theta^{b}\right\|_{\Theta_{T}}\right),
$$

where $K=4 C C^{\prime}(R)$ depends on $R$ but not on $T$. For $T \in\left(0, T_{0}\right)$ such that

$$
T<r / R \text { and } K T^{1 / 4} \leq 1 / 2,
$$

estimates (3.12) and (3.5) yield

$$
\left\|\Lambda^{T}\left(\tilde{\mathbf{u}}, \tilde{p}, \theta_{1}, \theta_{2}\right)\right\|_{\mathbb{N}_{T}} \leq\left\|\Lambda^{T}(0,0,0,0)\right\|_{\mathbb{N}_{T}}+K T^{1 / 4}\left(\|\tilde{\mathbf{u}}\|_{\mathbb{U}_{T}}+\|\tilde{p}\|_{\mathbb{P}_{T}}+\|\theta\|_{\Theta_{T}}\right) \leq R / 2+K R T^{1 / 4} \leq R .
$$

Then, $\forall T \in[0, T], \Lambda^{T}: \mathbb{B}_{R}(T) \rightarrow \mathbb{B}_{R}(T)$ and according to (3.12), the application $\Lambda^{T}$ is a contraction on $\mathbb{B}_{R}(T)$.

We have proven that $\Lambda^{T}: \mathbb{B}_{R}(T) \rightarrow \mathbb{B}_{R}(T)$ is a contraction. Then, according to the Picard fixed point theorem, there exists a unique fixed point to $\Lambda^{T}$ in $\mathbb{B}_{R}(T)$. This fixed point is the solution to problem (2.11) where the source terms are given by (3.1). This proves Theorem 3.1.

\subsection{Result in the moving domain, Theorem 1.8}

We consider $\left(\tilde{\mathbf{u}}, \tilde{p}, \theta_{1}, \theta_{2}\right)$ in $\mathbb{N}_{T}$ the solution to problem $(2.11)$ with (3.1) given by Theorem 3.1. Let $\mathbf{u}(t, \mathbf{x})=$ $\operatorname{cof}\left(\mathcal{J}_{\mathbf{\Psi}}\left(\theta_{1}(t), \theta_{2}(t), \mathbf{x}\right)\right)^{T} \tilde{\mathbf{u}}\left(t, \boldsymbol{\Psi}\left(\theta_{1}(t), \theta_{2}(t), \mathbf{x}\right)\right)$ and $p(t, \mathbf{x})=\tilde{p}\left(t, \boldsymbol{\Psi}\left(\theta_{1}(t), \theta_{2}(t), \mathbf{x}\right)\right)$. Then the quadruplet $\left(\mathbf{u}, p, \theta_{1}, \theta_{2}\right)$ is solution to the problem in the moving domain, i.e. problem (1.24). This proves Theorem 1.8.

Acknowledgement: The author would like to thank the anonymous referees who improved the presentation of the introduction.

\section{Appendix}

\section{A Proof of Lemma 1.2}

We have $g_{\theta_{1}}\left(y_{1}\right)+g_{\theta_{2}}\left(y_{1}\right)=y_{1}$, Assumption (1.4) is then fulfilled. The reference domain $S_{\text {ref }}$ is chosen to satisfy Assumption (1.5). Assumption (1.6) is fulfilled by restriction of $\mathbb{D}_{\Theta}$. All terms composing $\mathbf{X}^{b}$ in (1.2) are smooth, Assumption (1.8) is then easily fulfilled. For all $y_{1}<y_{P, 1}, g_{\theta_{2}}\left(y_{1}\right)=0$. This implies that $\forall y_{1}<y_{P, 1}, \partial_{\theta_{2}} \mathbf{X}^{b}\left(y_{1}, \theta_{1}, \theta_{2}\right)=0$, which proves (1.9).

We still have to show that $\mathbf{X}^{b}$ is a bijection. Let us compute its jacobian matrix. We denote the jacobian components as follows

$$
\mathcal{J}_{\mathbf{X}^{b}}=\left(\begin{array}{ll}
\partial_{1} X_{1} & \partial_{2} X_{1} \\
\partial_{1} X_{2} & \partial_{2} X_{2}
\end{array}\right)
$$

We have

$$
\begin{aligned}
& \operatorname{det}\left(\mathcal{J}_{\mathbf{X}^{b}}\right)=\partial_{1} X_{1} \times \partial_{2} X_{2}-\partial_{2} X_{1} \times \partial_{1} X_{2} \\
& =\left(g_{\theta_{1}}^{\prime} \mathbf{e}_{r 1}+g_{\theta_{2}}^{\prime} \mathbf{e}_{r 2}+y_{2}\left(\frac{\mathbf{N}^{\prime}}{\|\mathbf{N}\|}-\frac{(\|\mathbf{N}\|)^{\prime} \mathbf{N}}{\|\mathbf{N}\|^{2}}\right)\right)_{1} \times\left(\frac{\mathbf{N}}{\|\mathbf{N}\|}\right)_{2} \\
& -\left(g_{\theta_{1}}^{\prime} \mathbf{e}_{r 1}+g_{\theta_{2}}^{\prime} \mathbf{e}_{r 2}+y_{2}\left(\frac{\mathbf{N}^{\prime}}{\|\mathbf{N}\|}-\frac{(\|\mathbf{N}\|)^{\prime} \mathbf{N}}{\|\mathbf{N}\|^{2}}\right)\right)_{2} \times\left(\frac{\mathbf{N}}{\|\mathbf{N}\|}\right)_{1} \\
& =\left(g_{\theta_{1}}^{\prime} \mathbf{e}_{r 1}+g_{\theta_{2}}^{\prime} \mathbf{e}_{r 2}+y_{2} \frac{\mathbf{N}^{\prime}}{\|\mathbf{N}\|}\right)_{1} \times\left(\frac{\mathbf{N}}{\|\mathbf{N}\|}\right)_{2}-\left(g_{\theta_{1}}^{\prime} \mathbf{e}_{r 1}+g_{\theta_{2}}^{\prime} \mathbf{e}_{r 2}+y_{2} \frac{\mathbf{N}^{\prime}}{\|\mathbf{N}\|}\right)_{2} \times\left(\frac{\mathbf{N}}{\|\mathbf{N}\|}\right)_{1} \\
& =\left(g_{\theta_{1}}^{\prime} \cos \left(\theta_{1}\right)+g_{\theta_{2}}^{\prime} \cos \left(\theta_{1}+\theta_{2}\right)+y_{2} \frac{-g_{\theta_{1}}^{\prime \prime} \sin \left(\theta_{1}\right)-g_{\theta_{2}}^{\prime \prime} \sin \left(\theta_{1}+\theta_{2}\right)}{\|\mathbf{N}\|}\right) \times \frac{g_{\theta_{1}}^{\prime} \cos \left(\theta_{1}\right)+g_{\theta_{2}}^{\prime} \cos \left(\theta_{1}+\theta_{2}\right)}{\|\mathbf{N}\|} \\
& -\left(g_{\theta_{1}}^{\prime} \sin \left(\theta_{1}\right)+g_{\theta_{2}}^{\prime} \sin \left(\theta_{1}+\theta_{2}\right)+y_{2} \frac{g_{\theta_{1}}^{\prime \prime} \cos \left(\theta_{1}\right)+g_{\theta_{2}}^{\prime \prime} \cos \left(\theta_{1}+\theta_{2}\right)}{\|\mathbf{N}\|}\right) \times \frac{-g_{\theta_{1}}^{\prime} \sin \left(\theta_{1}\right)-g_{\theta_{2}}^{\prime} \sin \left(\theta_{1}+\theta_{2}\right)}{\|\mathbf{N}\|} \\
& =\frac{1}{\|\mathbf{N}\|}\left(\left(g_{\theta_{1}}^{\prime}\right)^{2}+\left(g_{\theta_{2}}^{\prime}\right)^{2}+2 g_{\theta_{1}}^{\prime} g_{\theta_{2}}^{\prime}\left(\sin \left(\theta_{1}\right) \sin \left(\theta_{1}+\theta_{2}\right)+\cos \left(\theta_{1}\right) \cos \left(\theta_{1}+\theta_{2}\right)\right)\right) \\
& +\frac{y_{2}}{\|\mathbf{N}\|^{2}}\left(g_{\theta_{1}}^{\prime \prime} g_{\theta_{2}}^{\prime}\left(\cos \left(\theta_{1}\right) \sin \left(\theta_{1}+\theta_{2}\right)-\sin \left(\theta_{1}\right) \cos \left(\theta_{1}+\theta_{2}\right)\right)\right. \\
& \left.+g_{\theta_{1}}^{\prime} g_{\theta_{2}}^{\prime \prime}\left(\cos \left(\theta_{1}+\theta_{2}\right) \sin \left(\theta_{1}\right)-\cos \left(\theta_{1}\right) \sin \left(\theta_{1}+\theta_{2}\right)\right)\right)
\end{aligned}
$$




$$
\begin{aligned}
\operatorname{det}\left(\mathcal{J}_{\mathbf{X}^{b}}\right) & =\frac{1}{\|\mathbf{N}\|}\left(\left(g_{\theta_{1}}^{\prime}\right)^{2}+\left(g_{\theta_{2}}^{\prime}\right)^{2}+2 g_{\theta_{1}}^{\prime} g_{\theta_{2}}^{\prime} \cos \left(\theta_{2}\right)\right)+\frac{y_{2}}{\|\mathbf{N}\|^{2}}\left(g_{\theta_{1}}^{\prime \prime} g_{\theta_{2}}^{\prime} \sin \left(\theta_{2}\right)+g_{\theta_{2}}^{\prime \prime} g_{\theta_{1}}^{\prime} \sin \left(-\theta_{2}\right)\right) \\
& =\|\mathbf{N}\|+\frac{y_{2}}{\|\mathbf{N}\|^{2}}\left(g_{\theta_{1}}^{\prime \prime} g_{\theta_{2}}^{\prime}-g_{\theta_{2}}^{\prime \prime} g_{\theta_{1}}^{\prime}\right) \sin \left(\theta_{2}\right) .
\end{aligned}
$$

We consider $\widetilde{S_{\text {ref }}}$ such that $\cup_{\mathbf{y} \in S_{\text {ref }}} B\left(\mathbf{y}, \varepsilon^{\prime}\right) \subset \widetilde{S_{\text {ref }}}$ for some $\varepsilon^{\prime}>0$. Since $\|\mathbf{N}\| \geq \alpha^{\prime}$ for some $\alpha^{\prime}>0$, we can choose $\theta_{2}$ small enough, i.e. $\mathbb{D}_{\Theta}$ small enough, such that $\operatorname{det}\left(\mathcal{J}_{\mathbf{X}^{b}}\left(\theta_{1}, \theta_{2}, \mathbf{y}\right)\right)>0$ for every $\mathbf{y} \in \widetilde{S_{\text {ref }}}$. According to the inverse function Theorem, the function $\mathbf{X}^{b}\left(\theta_{1}, \theta_{2},.\right)$ is then locally invertible, i.e. for every $\mathbf{y} \in \widetilde{S_{\text {ref }}}$, there exists $\varepsilon_{\mathbf{y}}>0$ such that $\mathbf{X}^{b}\left(\theta_{1}, \theta_{2},.\right)$ is invertible on $B\left(\mathbf{y}, \varepsilon_{\mathbf{y}}\right)$. We consider $\varepsilon=\min _{\mathbf{y} \in \overline{S_{\text {ref }}}} \varepsilon_{\mathbf{y}}>0$ which is defined since $\overline{S_{\text {ref }}}$ is closed. We established

$$
\forall \mathbf{y} \in S_{\mathrm{ref}}, \mathbf{X}^{b}\left(\theta_{1}, \theta_{2}, .\right) \text { is invertible on } B(\mathbf{y}, \varepsilon) .
$$

We end with a proof by contradiction. Assume that there exist $\mathbf{y}, \mathbf{y}^{\prime} \in S_{\text {ref }}$ such that $\mathbf{X}^{b}\left(\theta_{1}, \theta_{2}, \mathbf{y}\right)=$ $\mathbf{X}^{b}\left(\theta_{1}, \theta_{2}, \mathbf{y}^{\prime}\right)$. We have

$$
\begin{aligned}
\left\|\mathbf{y}-\mathbf{y}^{\prime}\right\| & \leq\left\|\mathbf{X}^{b}(0,0, \mathbf{y})-\mathbf{X}^{b}\left(0,0, \mathbf{y}^{\prime}\right)\right\| \\
& \leq\left\|\mathbf{X}^{b}(0,0, \mathbf{y})-\mathbf{X}^{b}\left(\theta_{1}, \theta_{2}, \mathbf{y}\right)\right\|+\left\|\mathbf{X}^{b}\left(0,0, \mathbf{y}^{\prime}\right)-\mathbf{X}^{b}\left(\theta_{1}, \theta_{2}, \mathbf{y}^{\prime}\right)\right\| \\
& \leq 2\left\|\nabla_{\theta_{1}, \theta_{2}} \mathbf{X}^{b}\right\|_{\mathbf{L}^{\infty}\left(\mathbb{D}_{\Theta} \times \Omega\right)}\left\|\left(\theta_{1}, \theta_{2}\right)\right\| .
\end{aligned}
$$

Then, for $\mathbb{D}_{\Theta}$ small enough, we have $\left\|\mathbf{y}-\mathbf{y}^{\prime}\right\| \leq \varepsilon / 2$, which is in contradiction with (A.1).

\section{B Proof of Lemma 3.2}

This section is devoted to the proof of Lemma 3.2. We start with some intermediate lemmas that will be used to decompose the intricate terms of Lemma 3.2 in smaller pieces.

\section{B.1 Technical Lemmas}

The following lemma contains Lipschitz estimates on several terms.

Lemma B.1. For $R>0$, there exists a constant $C=C(R)>0$ such that for every $T \in\left(0, T_{0}\right)$ and every $\left(., ., \theta_{1}^{j}, \theta_{2}^{j}\right) \in \mathbb{B}_{R}(T)$, the following estimates hold

$$
\begin{aligned}
& \left\|\mathbf{\Phi}\left(\theta_{1}^{a}, \theta_{2}^{a}\right)-\mathbf{\Phi}\left(\theta_{1}^{b}, \theta_{2}^{b}\right)\right\|_{L^{\infty}\left(0, T ; \mathbf{L}^{\infty}(\Omega)\right)} \leq C T\left\|\theta^{a}-\theta^{b}\right\|_{\Theta_{T}}, \\
& \left\|\mathcal{J}_{\boldsymbol{\Phi}}\left(\theta_{1}^{a}, \theta_{2}^{a}\right)-\mathcal{J}_{\boldsymbol{\Phi}}\left(\theta_{1}^{b}, \theta_{2}^{b}\right)\right\|_{L^{\infty}\left(0, T ; \mathbf{H}^{2}(\Omega)\right)} \leq C T\left\|\theta^{a}-\theta^{b}\right\|_{\Theta_{T}}, \\
& \left\|\mathcal{J}_{\Psi}\left(\theta_{1}^{a}, \theta_{2}^{a}, \boldsymbol{\Phi}^{a}\right)-\mathcal{J}_{\Psi}\left(\theta_{1}^{b}, \theta_{2}^{b}, \mathbf{\Phi}^{b}\right)\right\|_{L^{\infty}\left(0, T ; \mathbf{H}^{2}(\Omega)\right)} \leq C T\left\|\theta^{a}-\theta^{b}\right\|_{\Theta_{T}}, \\
& \left\|\left(\partial_{x_{j}} \mathcal{J}_{\Psi}\left(\theta_{1}^{a}, \theta_{2}^{a}\right)\right) \circ \mathbf{\Phi}\left(\theta_{1}^{a}, \theta_{2}^{a}\right)-\left(\partial_{x_{j}} \mathcal{J}_{\mathbf{\Psi}}\left(\theta_{1}^{b}, \theta_{2}^{b}\right)\right) \circ \mathbf{\Phi}\left(\theta_{1}^{b}, \theta_{2}^{b}\right)\right\|_{L^{\infty}\left(0, T ; \mathbf{H}^{1}(\Omega)\right)} \leq C T\left\|\theta^{a}-\theta^{b}\right\|_{\Theta_{T}}, \\
& \left\|\left(\partial_{x_{j}}^{2} \mathcal{J}_{\Psi}\left(\theta_{1}^{a}, \theta_{2}^{a}\right)\right) \circ \mathbf{\Phi}\left(\theta_{1}^{a}, \theta_{2}^{a}\right)-\left(\partial_{x_{j}}^{2} \mathcal{J}_{\Psi}\left(\theta_{1}^{b}, \theta_{2}^{b}\right)\right) \circ \mathbf{\Phi}\left(\theta_{1}^{b}, \theta_{2}^{b}\right)\right\|_{L^{\infty}\left(0, T ; \mathbf{L}^{2}(\Omega)\right)} \leq C T\left\|\theta^{a}-\theta^{b}\right\|_{\Theta_{T}}, \\
& \left\|\mathcal{M}_{\theta_{1}^{a}, \theta_{2}^{a}}-\mathcal{M}_{\theta_{1}^{b}, \theta_{2}^{b}}\right\|_{L^{\infty}(0, T)} \leq C T\left\|\theta^{a}-\theta^{b}\right\|_{\Theta_{T}}, \\
& \left\|\mathbf{n}_{\theta_{1}^{a}, \theta_{2}^{a}}\left(\mathbf{\Phi}\left(\theta_{1}^{a}, \theta_{2}^{a}\right)\right)-\mathbf{n}_{\theta_{1}^{b}, \theta_{2}^{b}}\left(\mathbf{\Phi}\left(\theta_{1}^{b}, \theta_{2}^{b}\right)\right)\right\|_{L^{\infty}\left(0, T ; \mathbf{L}^{\infty}\left(\partial S_{0}\right)\right)} \leq C T\left\|\theta^{a}-\theta^{b}\right\|_{\Theta_{T}}, \\
& \left\|\operatorname{det}\left(\mathcal{J}_{\Psi}\left(\theta_{1}^{a}, \theta_{2}^{a}\right)\right)-\operatorname{det}\left(\mathcal{J}_{\Psi}\left(\theta_{1}^{b}, \theta_{2}^{b}\right)\right)\right\|_{L^{\infty}\left(0, T ; \mathbf{L}^{\infty}(\Omega)\right)} \leq C T\left\|\theta^{a}-\theta^{b}\right\|_{\Theta_{T}}, \\
& \left\|\partial_{\theta_{j}} \mathbf{\Phi}\left(\theta_{1}^{a}, \theta_{2}^{a}, .\right)-\partial_{\theta_{j}} \mathbf{\Phi}\left(\theta_{1}^{b}, \theta_{2}^{b}, .\right)\right\|_{L^{\infty}\left(0, T ; \mathbf{H}^{2}(\Omega)\right)} \leq C T\left\|\theta^{a}-\theta^{b}\right\|_{\Theta_{T}}, \\
& \left\|\partial_{\theta_{k} \theta_{j}} \mathbf{\Phi}\left(\theta_{1}^{a}, \theta_{2}^{a}, .\right)-\partial_{\theta_{k} \theta_{j}} \mathbf{\Phi}\left(\theta_{1}^{b}, \theta_{2}^{b}, .\right)\right\|_{L^{\infty}\left(0, T ; \mathbf{H}^{2}(\Omega)\right)} \leq C T\left\|\theta^{a}-\theta^{b}\right\|_{\Theta_{T}}, \\
& \left\|\left|\mathcal{J}_{\boldsymbol{\Phi}}\left(\theta_{1}^{a}, \theta_{2}^{a}\right) \mathbf{t}_{0}\right|-\left|\mathcal{J}_{\boldsymbol{\Phi}}\left(\theta_{1}^{b}, \theta_{2}^{b}\right) \mathbf{t}_{0}\right|\right\|_{L^{\infty}\left(0, T ; L^{\infty}\left(\partial S_{0}\right)\right)} \leq C T\left\|\theta^{a}-\theta^{b}\right\|_{\Theta_{T}},
\end{aligned}
$$

and

$$
\begin{aligned}
&\left\|\partial_{t} \mathcal{J}_{\boldsymbol{\Phi}}\left(\theta_{1}^{a}, \theta_{2}^{a}\right)-\partial_{t} \mathcal{J}_{\mathbf{\Phi}}\left(\theta_{1}^{b}, \theta_{2}^{b}\right)\right\|_{L^{\infty}\left(0, T ; \mathbf{H}^{2}(\Omega)\right)} \leq C\left\|\theta^{a}-\theta^{b}\right\|_{\Theta_{T}}, \\
&\left\|\partial_{t}\left(\mathbf{\Psi}\left(\theta_{1}^{a}, \theta_{2}^{a}\right)\right) \circ \mathbf{\Phi}\left(\theta_{1}^{a}, \theta_{2}^{a}\right)-\partial_{t}\left(\mathbf{\Psi}\left(\theta_{1}^{b}, \theta_{2}^{b}\right)\right) \circ \mathbf{\Phi}\left(\theta_{1}^{b}, \theta_{2}^{b}\right)\right\|_{L^{\infty}\left(0, T ; \mathbf{L}^{\infty}(\Omega)\right)} \leq C\left\|\theta^{a}-\theta^{b}\right\|_{\Theta_{T}}, \\
& \| \partial_{t}\left(\mathcal{J}_{\mathbf{\Psi}}\left(\theta_{1}^{a}, \theta_{2}^{a}\right)\right) \circ \mathbf{\Phi}\left(\theta_{1}^{a}, \theta_{2}^{a}\right)-\partial_{t}\left(\mathcal{J}_{\mathbf{\Psi}}\left(\theta_{1}^{b}, \theta_{2}^{b}\right) \circ \circ \mathbf{\Phi}\left(\theta_{1}^{b}, \theta_{2}^{b}\right)\left\|_{L^{\infty}\left(0, T ; \mathbf{L}^{\infty}(\Omega)\right)} \leq C\right\| \theta^{a}-\theta^{b} \|_{\Theta_{T}},\right. \\
&\left\|\partial_{t}\left(\partial_{\theta_{j}} \mathbf{\Phi}\left(\theta_{1}^{a}, \theta_{2}^{a}, .\right)\right)-\partial_{t}\left(\partial_{\theta_{j}} \mathbf{\Phi}\left(\theta_{1}^{b}, \theta_{2}^{b}, .\right)\right)\right\|_{L^{\infty}\left(0, T ; \mathbf{H}^{2}\left(\mathscr{F}_{0}\right)\right)} \leq C\left\|\theta^{a}-\theta^{b}\right\|_{\Theta_{T}}
\end{aligned}
$$

Moreover, for every $\left(\tilde{\mathbf{u}}^{j}, ., \theta_{1}^{j}, \theta_{2}^{j}\right) \in \mathbb{B}_{R}(T)$, the following estimates hold on $\mathcal{G}$ defined in (3.3)

$$
\begin{array}{r}
\left\|\mathcal{G}\left(\theta_{1}^{a}, \theta_{2}^{a}, \tilde{\mathbf{u}}^{a}\right)-\mathcal{G}\left(\theta_{1}^{b}, \theta_{2}^{b}, \tilde{\mathbf{u}}^{b}\right)\right\|_{L^{2}\left(0, T ; \mathbf{L}^{2}\left(\partial S_{0}\right)\right)} \leq C\left(\left\|\theta^{a}-\theta^{b}\right\|_{\Theta_{T}}+\left\|\tilde{\mathbf{u}}^{a}-\tilde{\mathbf{u}}^{b}\right\|_{\mathbb{U}_{T}}\right), \\
\left\|\nabla \tilde{\mathbf{u}}^{a}-\mathcal{G}\left(\theta_{1}^{a}, \theta_{2}^{a}, \tilde{\mathbf{u}}^{a}\right)-\nabla \tilde{\mathbf{u}}^{b}+\mathcal{G}\left(\theta_{1}^{b}, \theta_{2}^{b}, \tilde{\mathbf{u}}^{b}\right)\right\|_{L^{2}\left(0, T ; \mathbf{L}^{2}\left(\partial S_{0}\right)\right)} \leq C T\left(\left\|\theta^{a}-\theta^{b}\right\|_{\Theta_{T}}+\left\|\tilde{\mathbf{u}}^{a}-\tilde{\mathbf{u}}^{b}\right\|_{\mathbf{U}_{T}}\right) .
\end{array}
$$


In particular, as a direct application of Lemma B.1, using that $(0,0,0,0) \in \mathbb{B}_{R}(T)$, we obtain the following lemma.

Lemma B.2. For $R>0$, there exists a constant $C=C(R)>0$, such that for every $T \in\left(0, T_{0}\right)$ and every $\left(., ., \theta_{1}, \theta_{2}\right) \in \mathbb{B}_{R}(T)$, the following estimates hold

$$
\begin{aligned}
&\left\|\mathcal{J}_{\mathbf{\Phi}}\left(\theta_{1}, \theta_{2}\right)-I\right\|_{L^{\infty}\left(0, T ; \mathbf{H}^{2}(\Omega)\right)} \leq C T, \\
&\left\|\mathcal{J}_{\Psi}\left(\theta_{1}, \theta_{2}, \mathbf{\Phi}\left(\theta_{1}, \theta_{2}\right)\right)-I\right\|_{L^{\infty}\left(0, T ; \mathbf{H}^{2}(\Omega)\right)} \leq C T, \\
&\left\|\partial_{x_{j}} \mathcal{J}_{\Psi}\left(\theta_{1}, \theta_{2}\right) \circ \boldsymbol{\Phi}\left(\theta_{1}, \theta_{2}\right)\right\|_{L^{\infty}\left(0, T ; \mathbf{H}^{1}(\Omega)\right)} \leq C T, \\
&\left\|\partial_{x_{j}}^{2} \mathcal{J}_{\Psi}\left(\theta_{1}, \theta_{2}\right) \circ \boldsymbol{\Phi}\left(\theta_{1}, \theta_{2}\right)\right\|_{L^{\infty}\left(0, T ; \mathbf{L}^{2}(\Omega)\right)} \leq C T, \\
&\left\|\mathcal{M}_{\theta_{1}, \theta_{2}}-\mathcal{M}_{0,0}\right\|_{L^{\infty}(0, T)} \leq C T, \\
&\left\|\mathbf{n}_{\theta_{1}, \theta_{2}}\left(\mathbf{\Phi}\left(\theta_{1}, \theta_{2}\right)\right)-\mathbf{n}_{0}\right\|_{L^{\infty}\left(0, T ; \mathbf{L}^{\infty}\left(\partial S_{0}\right)\right)} \leq C T, \\
&\left\|\mid \mathcal{J}_{\boldsymbol{\Phi}} \mathbf{t}_{0}-1\right\|_{L^{\infty}\left(0, T ; L^{\infty}\left(\partial S_{0}\right)\right)} \leq C T,
\end{aligned}
$$

and

$$
\begin{aligned}
\left\|\partial_{t}\left(\mathcal{J}_{\mathbf{\Phi}}\left(\theta_{1}, \theta_{2}\right)\right)\right\|_{L^{\infty}\left(0, T ; \mathbf{H}^{2}(\Omega)\right)} & \leq C, \\
\left\|\frac{\partial}{\partial t}\left(\mathbf{\Psi}\left(\theta_{1}, \theta_{2}\right)\right) \circ \boldsymbol{\Phi}\left(\theta_{1}, \theta_{2}\right)\right\|_{L^{\infty}\left(0, T ; \mathbf{L}^{\infty}(\Omega)\right)} & \leq C, \\
\left\|\frac{\partial}{\partial t}\left(\mathcal{J}_{\Psi}\left(\theta_{1}, \theta_{2}\right)\right) \circ \boldsymbol{\Phi}\left(\theta_{1}, \theta_{2}\right)\right\|_{L_{\left(0, T ; \mathbf{L}^{\infty}(\Omega)\right)}} & \leq C .
\end{aligned}
$$

Moreover, for every $\left(\tilde{\mathbf{u}}, ., \theta_{1}, \theta_{2}\right) \in \mathbb{B}_{R}(T)$, we have the following estimate on $\mathcal{G}$

$$
\left\|\nabla \tilde{\mathbf{u}}-\mathcal{G}\left(\theta_{1}, \theta_{2}, \tilde{\mathbf{u}}\right)\right\|_{L^{2}\left(0, T ; \mathbf{L}^{2}\left(\partial S_{0}\right)\right)} \leq C T \text {. }
$$

Proof of Lemma B.1. Three kinds of estimates have to be proven. First estimates (B.1)-(B.10) are of the type

$$
\left\|\alpha\left(\theta_{1}^{a}, \theta_{2}^{a}\right)-\alpha\left(\theta_{1}^{b}, \theta_{2}^{b}\right)\right\|_{L^{\infty}(0, T ; \mathbb{X})} \leq C T\left\|\left(\theta_{1}^{a}, \theta_{2}^{a}\right)-\left(\theta_{1}^{b}, \theta_{2}^{b}\right)\right\|_{\Theta_{T}},
$$

where $\alpha$ is a differentiable function defined on $\mathbb{D}_{\Theta}$ and valued in $\mathbb{X}$. We thus use Taylor series and get

$$
\left\|\alpha\left(\theta_{1}^{a}, \theta_{2}^{a}\right)-\alpha\left(\theta_{1}^{b}, \theta_{2}^{b}\right)\right\|_{L^{\infty}(0, T ; \mathbb{X})} \leq \sup _{\left(\theta_{1}, \theta_{2}\right) \in \mathbb{D}_{\Theta}}\left\|\nabla_{\theta} \alpha\left(\theta_{1}, \theta_{2}\right)\right\|_{L^{\infty}(0, T ; \mathbb{X})}\left\|\theta^{a}-\theta^{b}\right\|_{L^{\infty}(0, T)} .
$$

According to the definition of $\mathrm{B}_{R}(T)$ in $(3.6), \theta^{a}(0)=\theta^{b}(0)=(0,0)$, we finish with

$$
\left\|\theta^{a}-\theta^{b}\right\|_{L^{\infty}(0, T)} \leq T\left\|\theta^{a}-\theta^{b}\right\|_{\Theta_{T}} .
$$

The second type of estimates (B.12)-(B.15) is of the form

$$
\left\|\alpha\left(\theta_{1}^{a}, \theta_{2}^{a}, \dot{\theta}_{1}^{a}, \dot{\theta}_{2}^{a}\right)-\alpha\left(\theta_{1}^{b}, \theta_{2}^{b}, \dot{\theta}_{1}^{b}, \dot{\theta}_{2}^{b}\right)\right\|_{L^{\infty}(0, T ; \mathbf{X})} \leq C\left\|\left(\theta_{1}^{a}, \theta_{2}^{a}\right)-\left(\theta_{1}^{b}, \theta_{2}^{b}\right)\right\|_{\Theta_{T}},
$$

where $\alpha$ is now a function defined on $\mathbb{D}_{\Theta} \times \mathbb{R}^{2}$ with values in $\mathbb{X}$. We use the same strategy and get $\left\|\alpha\left(\theta_{1}^{a}, \theta_{2}^{a}, \dot{\theta}_{1}^{a}, \dot{\theta}_{2}^{a}\right)-\alpha\left(\theta_{1}^{b}, \theta_{2}^{b}, \dot{\theta}_{1}^{b}, \dot{\theta}_{2}^{b}\right)\right\|_{L^{\infty}(0, T ; \mathbb{X})}$

$$
\leq \sup _{\substack{\left(\theta_{1}, \theta_{2}\right) \in \mathbb{D}_{\Theta} \\\left|\omega_{1}\right|+\left|\omega_{2}\right| \leq R}}\left\|\nabla_{\theta, \omega} \alpha\left(\theta_{1}, \theta_{2}, \omega_{1}, \omega_{2}\right)\right\|_{L^{\infty}(0, T ; \mathbb{X})}\left(\left\|\theta^{a}-\theta^{b}\right\|_{L^{\infty}(0, T)}+\left\|\dot{\theta}^{a}-\dot{\theta}^{b}\right\|_{L^{\infty}(0, T)}\right) .
$$

Note that contrary to the first type of estimates, we do not have the decay in $T$ because we did not enforce $\dot{\theta}^{a}(0)=\dot{\theta}^{b}(0)$.

Estimate (B.16) is a direct consequence of (B.17). The last estimate to prove is (B.17). We do it via the computation

$$
\begin{aligned}
\left(\mathcal{G}\left(\theta_{1}^{a}, \theta_{2}^{a}, \tilde{\mathbf{u}}^{a}\right)-\right. & \left.\mathcal{G}\left(\theta_{1}^{b}, \theta_{2}^{b}, \tilde{\mathbf{u}}^{b}\right)-\nabla \tilde{\mathbf{u}}^{a}+\nabla \tilde{\mathbf{u}}^{b}\right)_{i j} \\
& =\sum_{k}\left(\operatorname{cof}\left(\partial_{x_{j}} \mathcal{J}_{\Psi}\left(\theta_{1}^{a}, \theta_{2}^{a}, .\right) \circ \boldsymbol{\Phi}^{a}\right)_{k i}-\operatorname{cof}\left(\partial_{x_{j}} \mathcal{J}_{\Psi}\left(\theta_{1}^{b}, \theta_{2}^{b}, .\right) \circ \boldsymbol{\Phi}^{b}\right)_{k i}\right) \tilde{u}_{k}^{a} \\
& +\sum_{k} \operatorname{cof}\left(\partial_{x_{j}} \mathcal{J}_{\Psi}\left(\theta_{1}^{b}, \theta_{2}^{b}, .\right) \circ \boldsymbol{\Phi}^{b}\right)_{k i}\left(\tilde{u}_{k}^{a}-\tilde{u}_{k}^{b}\right) \\
& +\sum_{k, \ell} \operatorname{cof}\left(\mathcal{J}_{\Psi}\left(\theta_{1}^{a}, \theta_{2}^{a}, \boldsymbol{\Phi}^{a}\right)-\mathcal{J}_{\Psi}\left(\theta_{1}^{b}, \theta_{2}^{b}, \boldsymbol{\Phi}^{b}\right)\right)_{k i} \frac{\partial \tilde{u}_{k}^{a}}{\partial y_{\ell}} \frac{\partial \Psi_{\ell}}{\partial x_{j}}\left(\theta_{1}^{a}, \theta_{2}^{a}, \boldsymbol{\Phi}^{a}\right) \\
& +\sum_{k, \ell}\left(\operatorname{cof}\left(\mathcal{J}_{\Psi}\left(\theta_{1}^{b}, \theta_{2}^{b}, \boldsymbol{\Phi}^{b}\right)\right)_{k i} \frac{\partial \Psi_{\ell}}{\partial x_{j}}\left(\theta_{1}^{a}, \theta_{2}^{a}, \boldsymbol{\Phi}^{a}\right)-\delta_{k i} \delta_{\ell j}\right)\left(\frac{\partial \tilde{u}_{k}^{a}}{\partial y_{\ell}}-\frac{\partial \tilde{u}_{k}^{b}}{\partial y_{\ell}}\right) \\
& +\sum_{k, \ell} \operatorname{cof}\left(\mathcal{J}_{\Psi}\left(\theta_{1}^{b}, \theta_{2}^{b}, \boldsymbol{\Phi}^{b}\right)\right)_{k i} \frac{\partial \tilde{u}_{k}^{b}}{\partial y_{\ell}}\left(\frac{\partial \Psi_{\ell}}{\partial x_{j}}\left(\theta_{1}^{a}, \theta_{2}^{a}, \boldsymbol{\Phi}^{a}\right)-\frac{\partial \Psi_{\ell}}{\partial x_{j}}\left(\theta_{1}^{b}, \theta_{2}^{b}, \boldsymbol{\Phi}^{b}\right)\right),
\end{aligned}
$$

and with the use of estimates (B.3), (B.4), (B.19) and (B.20) we get estimate (B.17). 


\section{B.2 Detailed proof of Lemma 3.2}

Proof. In all the following estimates we use Lemmas B.1 and B.2.

- Estimate (3.7) is a consequence of the following estimates

$$
\begin{aligned}
& \left\|\mathbf{F}^{1}\left(\theta_{1}^{a}, \theta_{2}^{a}, \tilde{\mathbf{u}}^{a}\right)-\mathbf{F}^{1}\left(\theta_{1}^{b}, \theta_{2}^{b}, \tilde{\mathbf{u}}^{b}\right)\right\|_{\mathbb{F}_{T}} \leq C T\left(\left\|\theta^{a}-\theta^{b}\right\|_{\Theta_{T}}+\left\|\tilde{\mathbf{u}}^{a}-\tilde{\mathbf{u}}^{b}\right\|_{\mathrm{U}_{T}}\right), \\
& \left\|\mathbf{F}^{2}\left(\theta_{1}^{a}, \theta_{2}^{a}, \tilde{\mathbf{u}}^{a}\right)-\mathbf{F}^{2}\left(\theta_{1}^{b}, \theta_{2}^{b}, \tilde{\mathbf{u}}^{b}\right)\right\|_{\mathbb{F}_{T}} \leq C T^{1 / 2}\left(\left\|\theta^{a}-\theta^{b}\right\|_{\Theta_{T}}+\left\|\tilde{\mathbf{u}}^{a}-\tilde{\mathbf{u}}^{b}\right\|_{\mathrm{U}_{T}}\right), \\
& \left\|\mathbf{F}^{3}\left(\theta_{1}^{a}, \theta_{2}^{a}, \tilde{\mathbf{u}}^{a}\right)-\mathbf{F}^{3}\left(\theta_{1}^{b}, \theta_{2}^{b}, \tilde{\mathbf{u}}^{b}\right)\right\|_{\mathbb{F}_{T}} \leq C T\left(\left\|\theta^{a}-\theta^{b}\right\|_{\Theta_{T}}+\left\|\tilde{\mathbf{u}}^{a}-\tilde{\mathbf{u}}^{b}\right\|_{\mathbb{U}_{T}}\right), \\
& \left\|\mathbf{F}^{4}\left(\theta_{1}^{a}, \theta_{2}^{a}, \tilde{\mathbf{u}}^{a}\right)-\mathbf{F}^{4}\left(\theta_{1}^{b}, \theta_{2}^{b}, \tilde{\mathbf{u}}^{b}\right)\right\|_{\mathbb{F}_{T}} \leq C T^{1 / 4}\left(\left\|\theta^{a}-\theta^{b}\right\|_{\Theta_{T}}+\left\|\tilde{\mathbf{u}}^{a}-\tilde{\mathbf{u}}^{b}\right\|_{\mathrm{U}_{T}}\right), \\
& \left\|\mathbf{F}^{5}\left(\theta_{1}^{a}, \theta_{2}^{a}, \tilde{p}^{a}\right)-\mathbf{F}^{5}\left(\theta_{1}^{b}, \theta_{2}^{b}, \tilde{p}^{b}\right)\right\|_{\mathbb{F}_{T}} \leq C T\left(\left\|\theta^{a}-\theta^{b}\right\|_{\Theta_{T}}+\left\|\tilde{p}^{a}-\tilde{p}^{b}\right\|_{\mathbb{P}_{T}}\right),
\end{aligned}
$$

where $C$ does not depend on $T$. We now prove all of them.

- Estimate (B.29): We use the decomposition

$\mathbf{F}^{1}\left(\theta_{1}^{a}, \theta_{2}^{a}, \tilde{\mathbf{u}}^{a}\right)-\mathbf{F}^{1}\left(\theta_{1}^{b}, \theta_{2}^{b}, \tilde{\mathbf{u}}^{b}\right)=\left(I-\operatorname{cof}\left(\mathcal{J}_{\Psi}\left(\theta_{1}^{a}, \theta_{2}^{a}, \Phi^{a}\right)\right)^{T}\right)\left(\frac{\partial \tilde{\mathbf{u}}^{a}}{\partial t}-\frac{\partial \tilde{\mathbf{u}}^{b}}{\partial t}\right)+\operatorname{cof}\left(\mathcal{J}_{\Psi}\left(\theta_{1}^{b}, \theta_{2}^{b}, \Phi^{b}\right)-\mathcal{J}_{\Psi}\left(\theta_{1}^{a}, \theta_{2}^{a}, \Phi^{a}\right)\right)^{T} \frac{\partial \tilde{\mathbf{u}}^{b}}{\partial t}$, and we use estimates (B.19) and (B.3).

- Estimate (B.30): We use the decomposition

$$
\begin{aligned}
\mathbf{F}^{2}\left(\theta_{1}^{a}, \theta_{2}^{a}, \tilde{\mathbf{u}}^{a}\right)-\mathbf{F}^{2}\left(\theta_{1}^{b}, \theta_{2}^{b}, \tilde{\mathbf{u}}^{b}\right)= & -\operatorname{cof}\left(\frac{\partial}{\partial t}\left(\mathcal{J}_{\Psi}\left(\theta_{1}^{a}, \theta_{2}^{a}, .\right)\right) \circ \boldsymbol{\Phi}^{a}\right)^{T} \tilde{\mathbf{u}}^{a}+\operatorname{cof}\left(\frac{\partial}{\partial t}\left(\mathcal{J}_{\Psi}\left(\theta_{1}^{b}, \theta_{2}^{b}, .\right)\right) \circ \boldsymbol{\Phi}^{b}\right)^{T} \tilde{\mathbf{u}}^{b} \\
& +\operatorname{cof}\left(\mathcal{J}_{\Psi}\left(\theta_{1}^{b}, \theta_{2}^{b}, \boldsymbol{\Phi}^{b}\right)-\mathcal{J}_{\Psi}\left(\theta_{1}^{a}, \theta_{2}^{a}, \boldsymbol{\Phi}^{a}\right)\right)^{T} \nabla_{\mathbf{y}} \tilde{\mathbf{u}}^{a}\left(\frac{\partial}{\partial t} \mathbf{\Psi}\left(\theta_{1}^{a}, \theta_{2}^{a}, .\right)\right) \circ \boldsymbol{\Phi}^{a} \\
& +\operatorname{cof}\left(\mathcal{J}_{\Psi}\left(\theta_{1}^{b}, \theta_{2}^{b}, \boldsymbol{\Phi}^{b}\right)\right)^{T} \nabla_{\mathbf{y}}\left(\tilde{\mathbf{u}}^{b}-\tilde{\mathbf{u}}^{a}\right)\left(\frac{\partial}{\partial t} \boldsymbol{\Psi}\left(\theta_{1}^{a}, \theta_{2}^{a}, .\right)\right) \circ \boldsymbol{\Phi}^{a} \\
& +\operatorname{cof}\left(\mathcal{J}_{\Psi}\left(\theta_{1}^{b}, \theta_{2}^{b}, \boldsymbol{\Phi}^{b}\right)\right)^{T} \nabla_{\mathbf{y}} \tilde{\mathbf{u}}^{b}\left(\left(\frac{\partial}{\partial t} \boldsymbol{\Psi}\left(\theta_{1}^{b}, \theta_{2}^{b}, .\right)\right) \circ \boldsymbol{\Phi}^{b}-\left(\frac{\partial}{\partial t} \mathbf{\Psi}\left(\theta_{1}^{a}, \theta_{2}^{a}, .\right)\right) \circ \boldsymbol{\Phi}^{a}\right),
\end{aligned}
$$

where, for $j \in\{a, b\}$, we denote $\boldsymbol{\Phi}^{j}=\boldsymbol{\Phi}\left(\theta_{1}^{j}, \theta_{2}^{j}\right)$. We use estimates (B.27), (B.26), (B.14), (B.13), (B.3) and the estimate $\|\tilde{\mathbf{u}}\|_{L^{2}\left(0, T ; \mathbf{H}^{1}\left(\mathscr{F}_{0}\right)\right)} \leq T^{1 / 2}\|\tilde{\mathbf{u}}\|_{L^{\infty}\left(0, T ; \mathbf{H}^{1}\left(\mathscr{F}_{0}\right)\right)}$.

- Estimate (B.31): Since $\tilde{\mathbf{u}}$ has a space regularity of $\mathbf{H}_{\beta}^{2}\left(\mathscr{F}_{0}\right)$ and not $\mathbf{H}^{2}\left(\mathscr{F}_{0}\right)$, we need the following estimate in the sequel: for $f \in L^{\infty}\left(0, T ; L^{\infty}\left(\mathscr{F}_{0}\right)\right)$ with support within $\mathscr{F}_{0} \cap \Omega_{\varepsilon}$ (defined in Lemma 1.7), we have

$$
\left\|f \times \frac{\partial^{2} \tilde{u}_{k}}{\partial y_{\ell} \partial y_{m}}\right\|_{L^{2}\left(0, T ; L^{2}\left(\mathscr{F}_{0}\right)\right)} \leq C\|f\|_{L^{\infty}\left(0, T ; L^{\infty}\left(\mathscr{F}_{0}\right)\right)}\|\tilde{\mathbf{u}}\|_{L^{2}\left(0, T ; \mathbf{H}_{\beta}^{2}\left(\mathscr{F}_{0}\right)\right)},
$$

where $C$ does not depend on $T$. It is a consequence of

$$
\left\|f \times \frac{\partial^{2} \tilde{u}_{k}}{\partial y_{\ell} \partial y_{m}}\right\|_{L^{2}\left(0, T ; L^{2}\left(\mathscr{F}_{0}\right)\right)} \leq\left\|\frac{f}{\prod_{n \in \mathscr{J}_{d, n}} r_{n}^{\beta}}\right\|_{L^{\infty}\left(0, T ; L^{\infty}\left(\Omega_{\varepsilon} \cap \mathscr{F}_{0}\right)\right)}\left\|\prod_{n \in \mathscr{J}_{d, n}} r_{n}^{\beta} \frac{\partial^{2} \tilde{u}_{k}}{\partial y_{\ell} \partial y_{m}}\right\|_{L^{2}\left(0, T ; L^{2}\left(\mathscr{F}_{0}\right)\right)} .
$$

We now use the decomposition

$$
\left(\mathbf{F}^{3}\left(\theta_{1}^{a}, \theta_{2}^{a}, \tilde{\mathbf{u}}^{a}\right)-\mathbf{F}^{3}\left(\theta_{1}^{b}, \theta_{2}^{b}, \tilde{\mathbf{u}}^{b}\right)\right)_{i}=A_{1, i}+A_{2, i}+A_{3, i}+A_{4, i},
$$

where

$$
\begin{aligned}
A_{1, i}= & \nu \sum_{j, k, \ell, m}\left(\operatorname{cof}\left(\mathcal{J}_{\Psi}\left(\theta_{1}^{a}, \theta_{2}^{a}, \boldsymbol{\Phi}^{a}\right)\right)_{k i} \frac{\partial \Psi_{\ell}}{\partial x_{j}}\left(\theta_{1}^{a}, \theta_{2}^{a}, \mathbf{\Phi}^{a}\right) \frac{\partial \Psi_{m}}{\partial x_{j}}\left(\theta_{1}^{a}, \theta_{2}^{a}, \mathbf{\Phi}^{a}\right)\right. \\
& \left.-\operatorname{cof}\left(\mathcal{J}_{\Psi}\left(\theta_{1}^{b}, \theta_{2}^{b}, \boldsymbol{\Phi}^{b}\right)\right)_{k i} \frac{\partial \Psi_{\ell}}{\partial x_{j}}\left(\theta_{1}^{b}, \theta_{2}^{b}, \boldsymbol{\Phi}^{b}\right) \frac{\partial \Psi_{m}}{\partial x_{j}}\left(\theta_{1}^{b}, \theta_{2}^{b}, \boldsymbol{\Phi}^{b}\right)\right) \frac{\partial^{2} \tilde{u}_{k}^{a}}{\partial y_{\ell} \partial y_{m}} \\
& +\nu \sum_{j, k, \ell, m}\left(\operatorname{cof}\left(\mathcal{J}_{\Psi}\left(\theta_{1}^{b}, \theta_{2}^{b}, \boldsymbol{\Phi}^{b}\right)\right)_{k i} \frac{\partial \Psi_{\ell}}{\partial x_{j}}\left(\theta_{1}^{b}, \theta_{2}^{b}, \boldsymbol{\Phi}^{b}\right) \frac{\partial \Psi_{m}}{\partial x_{j}}\left(\theta_{1}^{b}, \theta_{2}^{b}, \boldsymbol{\Phi}^{b}\right)-\delta_{k i} \delta_{j \ell} \delta_{m j}\right)\left(\frac{\partial^{2} \tilde{u}_{k}^{a}}{\partial y_{\ell} \partial y_{m}}-\frac{\partial^{2} \tilde{u}_{k}^{b}}{\partial y_{\ell} \partial y_{m}}\right),
\end{aligned}
$$




$$
\begin{aligned}
A_{2, i}= & 2 \nu \sum_{j, k, \ell}\left(\operatorname{cof}\left(\partial_{x_{j}} \mathcal{J}_{\Psi}\left(\theta_{1}^{a}, \theta_{2}^{a}, \boldsymbol{\Phi}^{a}\right)\right)_{k i} \frac{\partial \Psi_{\ell}}{\partial x_{j}}\left(\theta_{1}^{a}, \theta_{2}^{a}, \boldsymbol{\Phi}^{a}\right)-\operatorname{cof}\left(\partial_{x_{j}} \mathcal{J}_{\Psi}\left(\theta_{1}^{b}, \theta_{2}^{b}, \boldsymbol{\Phi}^{b}\right)\right)_{k i} \frac{\partial \Psi_{\ell}}{\partial x_{j}}\left(\theta_{1}^{b}, \theta_{2}^{b}, \boldsymbol{\Phi}^{b}\right)\right) \frac{\partial \tilde{u}_{k}^{a}}{\partial y_{\ell}} \\
& +2 \nu \sum_{j, k, \ell} \operatorname{cof}\left(\partial_{x_{j}} \mathcal{J}_{\Psi}\left(\theta_{1}^{b}, \theta_{2}^{b}, \boldsymbol{\Phi}^{b}\right)\right)_{k i} \frac{\partial \Psi_{\ell}}{\partial x_{j}}\left(\theta_{1}^{b}, \theta_{2}^{b}, \boldsymbol{\Phi}^{b}\right)\left(\frac{\partial \tilde{u}_{k}^{a}}{\partial y_{\ell}}-\frac{\partial \tilde{u}_{k}^{b}}{\partial y_{\ell}}\right), \\
A_{3, i}= & \nu \sum_{j, k, \ell}\left(\operatorname{cof}\left(\mathcal{J}_{\Psi}\left(\theta_{1}^{a}, \theta_{2}^{a}, \Phi^{a}\right)\right)_{k i} \frac{\partial^{2} \Psi_{\ell}}{\partial x_{j}^{2}}\left(\theta_{1}^{a}, \theta_{2}^{a}, \boldsymbol{\Phi}^{a}\right)-\operatorname{cof}\left(\mathcal{J}_{\Psi}\left(\theta_{1}^{b}, \theta_{2}^{b}, \Phi^{b}\right)\right)_{k i} \frac{\partial^{2} \Psi_{\ell}}{\partial x_{j}^{2}}\left(\theta_{1}^{b}, \theta_{2}^{b}, \Phi^{b}\right)\right) \frac{\partial \tilde{u}_{k}^{a}}{\partial y_{\ell}} \\
& +\nu \sum_{j, k, \ell} \operatorname{cof}\left(\mathcal{J}_{\Psi}\left(\theta_{1}^{b}, \theta_{2}^{b}, \boldsymbol{\Phi}^{b}\right)\right)_{k i} \frac{\partial^{2} \Psi_{\ell}}{\partial x_{j}^{2}}\left(\theta_{1}^{b}, \theta_{2}^{b}, \boldsymbol{\Phi}^{b}\right)\left(\frac{\partial \tilde{u}_{k}^{a}}{\partial y_{\ell}}-\frac{\partial \tilde{u}_{k}^{a}}{\partial y_{\ell}}\right),
\end{aligned}
$$

and

$$
A_{4, i}=\nu \sum_{j, k}\left(\operatorname{cof}\left(\partial_{x_{j}}^{2} \mathcal{J}_{\Psi}\left(\theta_{1}^{a}, \theta_{2}^{a}, \mathbf{\Phi}^{a}\right)-\partial_{x_{j}}^{2} \mathcal{J}_{\Psi}\left(\theta_{1}^{b}, \theta_{2}^{b}, \mathbf{\Phi}^{b}\right)\right)_{k i} \tilde{u}_{k}^{a}+\operatorname{cof}\left(\partial_{x_{j}}^{2} \mathcal{J}_{\Psi}\left(\theta_{1}^{b}, \theta_{2}^{b}, \mathbf{\Phi}^{b}\right)\right)_{k i}\left(\tilde{u}_{k}^{a}-\tilde{u}_{k}^{a}\right)\right)
$$

Now,

- to estimate $A_{1, i}$, we use estimates (B.3), (B.19) and (B.34), also note that $\mathcal{J}_{\Psi}\left(\theta_{1}^{a}, \theta_{2}^{a}, \boldsymbol{\Phi}^{a}\right)-\mathcal{J}_{\Psi}\left(\theta_{1}^{b}, \theta_{2}^{b}, \Phi^{b}\right)$ has support within $\Omega_{\varepsilon}$.

- To estimate $A_{2, i}$, we use estimates (B.3), (B.4), (B.19) and (B.20).

- To estimate $A_{3, i}$, we use estimates (B.3), (B.4), (B.19) and (B.20).

- To estimate $A_{4, i}$, we use (B.5) and (B.21).

- Estimate (B.32): Let us first prove, for $f \in L^{\infty}\left(0, T ; H^{1}\left(\mathscr{F}_{0}\right)\right)$ and $g \in L^{\infty}\left(0, T ; L^{2}\left(\mathscr{F}_{0}\right)\right) \cap L^{2}\left(0, T ; H^{1 / 2}\left(\mathscr{F}_{0}\right)\right)$, the following estimate

$$
\|f g\|_{L^{2}\left(0, T ; L^{2}\left(\mathscr{F}_{0}\right)\right)} \leq C T^{1 / 4}\|f\|_{L^{\infty}\left(0, T ; H^{1}\left(\mathscr{F}_{0}\right)\right)}\|g\|_{L^{\infty}\left(0, T ; L^{2}\left(\mathscr{F}_{0}\right)\right)}^{1 / 2}\|g\|_{L^{2}\left(0, T ; H^{1 / 2}\left(\mathscr{F}_{0}\right)\right)}^{1 / 2},
$$

where $C$ does not depend on $T$.

- Hölder inequality with respect to time applied to $f g \times 1$ gives

$$
\|f g\|_{L^{2}\left(0, T ; L^{2}\left(\mathscr{F}_{0}\right)\right)} \leq T^{1 / 4}\|f g\|_{L^{4}\left(0, T ; L^{2}\left(\mathscr{F}_{0}\right)\right)} .
$$

- We have the relation $1 / 10+2 / 5=1 / 2$, then Hölder inequality yields

$$
\|f g\|_{L^{4}\left(0, T ; L^{2}\left(\mathscr{F}_{0}\right)\right)} \leq\|f\|_{L^{\infty}\left(0, T ; L^{10}\left(\mathscr{F}_{0}\right)\right)}\|g\|_{L^{4}\left(0, T ; L^{5 / 2}\left(\mathscr{F}_{0}\right)\right)} .
$$

- We have the Sobolev embedding $H^{1}\left(\mathscr{F}_{0}\right) \hookrightarrow L^{10}\left(\mathscr{F}_{0}\right):\|f\|_{L^{\infty}\left(0, T ; L^{10}\left(\mathscr{F}_{0}\right)\right)} \leq C\|f\|_{L^{\infty}\left(0, T ; H^{1}\left(\mathscr{F}_{0}\right)\right)}$.

- We have the Sobolev embedding $H^{1 / 4}\left(\mathscr{F}_{0}\right) \hookrightarrow L^{5 / 2}\left(\mathscr{F}_{0}\right)$ (see [1, Theorem 7.58]) and the Sobolev interpolation $\left[L^{2}\left(\mathscr{F}_{0}\right), H^{1 / 2}\left(\mathscr{F}_{0}\right)\right]_{1 / 2}=H^{1 / 4}\left(\mathscr{F}_{0}\right):\|g\|_{L^{5 / 2}\left(\mathscr{F}_{0}\right)} \leq C\|g\|_{H^{1 / 4}\left(\mathscr{F}_{0}\right)} \leq C\|g\|_{L^{2}\left(\mathscr{F}_{0}\right)}^{1 / 2}\|g\|_{H^{1 / 2}\left(\mathscr{F}_{0}\right)}^{1 / 2}$.

- We end the proof of (B.35) with

which implies

$$
\|g\|_{L^{4}\left(0, T ; L^{5 / 2}\left(\mathscr{F}_{0}\right)\right)}^{4}=\int_{0}^{T}\|g\|_{L^{5 / 2}\left(\mathscr{F}_{0}\right)}^{4} \leq C \int_{0}^{T}\|g\|_{L^{2}\left(\mathscr{F}_{0}\right)}^{2}\|g\|_{H^{1 / 2}\left(\mathscr{F}_{0}\right)}^{2},
$$

$$
\|g\|_{L^{4}\left(0, T ; L^{5 / 2}\left(\mathscr{F}_{0}\right)\right)} \leq C\|g\|_{L^{\infty}\left(0, T ; L^{2}\left(\mathscr{F}_{0}\right)\right)}^{1 / 2}\|g\|_{L^{2}\left(0, T ; H^{1 / 2}\left(\mathscr{F}_{0}\right)\right)}^{1 / 2} .
$$

We then use the decomposition

$$
\left(\mathbf{F}^{4}\left(\theta_{1}^{a}, \theta_{2}^{a}, \tilde{\mathbf{u}}^{a}\right)-\mathbf{F}^{4}\left(\theta_{1}^{b}, \theta_{2}^{b}, \tilde{\mathbf{u}}^{b}\right)\right)_{i}=B_{1, i}+B_{2, i}+B_{3, i}+B_{4, i}
$$

where

$$
\begin{aligned}
& B_{1, i}=-\sum_{j, k, r}\left(\operatorname{cof}\left(\mathcal{J}_{\Psi}\left(\theta_{1}^{a}, \theta_{2}^{a}, \Phi^{a}\right)\right)_{k j} \operatorname{cof}\left(\partial_{x_{j}} \mathcal{J}_{\Psi}\left(\theta_{1}^{a}, \theta_{2}^{a}, \Phi^{a}\right)\right)_{r i}-\operatorname{cof}\left(\mathcal{J}_{\Psi}\left(\theta_{1}^{b}, \theta_{2}^{b}, \Phi^{b}\right)\right)_{k j} \operatorname{cof}\left(\partial_{x_{j}} \mathcal{J}_{\Psi}\left(\theta_{1}^{b}, \theta_{2}^{b}, \Phi^{b}\right)\right)_{r i}\right) \tilde{u}_{k}^{a} \tilde{u}_{r}^{a}, \\
& B_{2, i}=-\sum_{j, k, r} \operatorname{cof}\left(\mathcal{J}_{\Psi}\left(\theta_{1}^{b}, \theta_{2}^{b}, \Phi^{b}\right)\right)_{k j} \operatorname{cof}\left(\partial_{x_{j}} \mathcal{J}_{\Psi}\left(\theta_{1}^{b}, \theta_{2}^{b}, \Phi^{b}\right)\right)_{r i}\left(\tilde{u}_{k}^{a} \tilde{u}_{r}^{a}-\tilde{u}_{k}^{b} \tilde{u}_{r}^{b}\right), \\
& B_{3, i}=-\sum_{k, r}\left(\operatorname{det}\left(\mathcal{J}_{\Psi}\left(\theta_{1}^{a}, \theta_{2}^{a}, \Phi^{a}\right)\right)^{2} \frac{\partial \Phi_{i}^{a}}{\partial y_{r}}-\operatorname{det}\left(\mathcal{J}_{\Psi}\left(\theta_{1}^{b}, \theta_{2}^{b}, \Phi^{b}\right)\right)^{2} \frac{\partial \Phi_{i}^{b}}{\partial y_{r}}\right) \tilde{u}_{k}^{a} \frac{\partial \tilde{u}_{r}^{a}}{\partial y_{k}} \\
& B_{4, i}=-\sum_{k, r} \operatorname{det}\left(\mathcal{J}_{\Psi}\left(\theta_{1}^{b}, \theta_{2}^{b}, \Phi^{b}\right)\right)^{2} \frac{\partial \Phi_{i}^{b}}{\partial y_{r}}\left(\tilde{u}_{k}^{a} \frac{\partial \tilde{u}_{r}^{a}}{\partial y_{k}}-\tilde{u}_{k}^{b} \frac{\partial \tilde{u}_{r}^{b}}{\partial y_{k}}\right) .
\end{aligned}
$$


We use

- estimates (B.3), (B.4), (B.19), (B.20) and (B.35) with $f=\tilde{u}_{k}^{a}, g=\tilde{u}_{r}^{a}$ for $B_{1, i}$,

- estimates (B.19), (B.20), (B.35) with $f=\tilde{u}_{k}^{a}, g=\tilde{u}_{r}^{a}-\tilde{u}_{r}^{b}$ and (B.35) with $f=\tilde{u}_{k}^{a}-\tilde{u}_{k}^{b}, g=\tilde{u}_{r}^{b}$ for $B_{2, i}$,

- estimates (B.3), (B.2), (B.18), (B.19), (1.28) and (B.35) with $f=\tilde{u}_{k}^{a}, g=\frac{\partial \tilde{u}_{r}^{a}}{\partial y_{k}}$ for $B_{3, i}$,

- estimates (B.18), (B.19), (1.28), (B.35) with $f=\tilde{u}_{k}^{a}, g=\frac{\partial \tilde{u}_{r}^{a}}{\partial y_{k}}-\frac{\partial \tilde{u}_{r}^{b}}{\partial y_{k}}$ and (B.35) with $f=\tilde{u}_{k}^{a}-\tilde{u}_{k}^{b}$, $g=\frac{\partial \tilde{u}_{r}^{b}}{\partial y_{k}}$ for $B_{4, i}$.

- Estimate (B.33): We have

$\mathbf{F}^{5}\left(\theta_{1}^{a}, \theta_{2}^{a}, \tilde{p}^{a}\right)-\mathbf{F}^{5}\left(\theta_{1}^{b}, \theta_{2}^{b}, \tilde{p}^{b}\right)=\left(I-\mathcal{J}_{\Psi}\left(\theta_{1}^{a}, \theta_{2}^{a}, \mathbf{\Phi}^{a}\right)\right)^{T} \nabla_{\mathbf{y}}\left(\tilde{p}^{a}-\tilde{p}^{b}\right)+\left(\mathcal{J}_{\Psi}\left(\theta_{1}^{b}, \theta_{2}^{b}, \mathbf{\Phi}^{b}\right)-\mathcal{J}_{\Psi}\left(\theta_{1}^{a}, \theta_{2}^{a}, \mathbf{\Phi}^{a}\right)\right)^{T} \nabla_{\mathbf{y}} \tilde{p}^{b}$, we use estimates (B.3), (B.19) and we adapt estimate (B.34) to obtain a $H_{\beta}^{1}\left(\mathscr{F}_{0}\right)$ norm for $\tilde{p}$.

- Estimate (3.8): We use, for $f \in L^{2}(0, T)$, the Hölder inequality

$$
\|f\|_{L^{2}(0, T)} \leq T^{1 / 2}\|f\|_{L^{\infty}(0, T)} .
$$

We have

$$
\frac{\partial}{\partial t}\left(\mathbf{G}\left(\theta_{1}^{a}, \theta_{2}^{a}, \dot{\theta}_{1}^{a}, \dot{\theta}_{2}^{a}\right)-\mathbf{G}\left(\theta_{1}^{b}, \theta_{2}^{b}, \dot{\theta}_{1}^{b}, \dot{\theta}_{2}^{b}\right)\right)=\mathbf{D}_{1}+\mathbf{D}_{2}+\mathbf{D}_{3}+\mathbf{D}_{4},
$$

where

$$
\begin{aligned}
& \mathbf{D}_{1}=\sum_{j}\left(\ddot{\theta}_{j}^{a}-\ddot{\theta}_{j}^{b}\right)\left(\operatorname{cof}\left(\mathcal{J}_{\boldsymbol{\Phi}}\left(\theta_{1}^{a}, \theta_{2}^{a}, \mathbf{y}\right)\right)^{T} \partial_{\theta_{j}} \mathbf{\Phi}\left(\theta_{1}^{a}, \theta_{2}^{a}, \mathbf{y}\right)-\partial_{\theta_{j}} \mathbf{\Phi}(0,0, \mathbf{y})\right) \\
& \mathbf{D}_{2}=\sum_{j} \ddot{\theta}_{j}^{b}\left(\operatorname{cof}\left(\mathcal{J}_{\mathbf{\Phi}}\left(\theta_{1}^{a}, \theta_{2}^{a}, \mathbf{y}\right)^{T} \partial_{\theta_{j}} \mathbf{\Phi}\left(\theta_{1}^{a}, \theta_{2}^{a}, \mathbf{y}\right)\right)-\operatorname{cof}\left(\mathcal{J}_{\mathbf{\Phi}}\left(\theta_{1}^{b}, \theta_{2}^{b}, \mathbf{y}\right)^{T} \partial_{\theta_{j}} \mathbf{\Phi}\left(\theta_{1}^{b}, \theta_{2}^{b}, \mathbf{y}\right)\right)\right), \\
& \mathbf{D}_{3}=\sum_{j}\left(\dot{\theta}_{j}^{a}-\dot{\theta}_{j}^{b}\right)\left(\operatorname{cof}\left(\partial_{t}\left(\mathcal{J}_{\mathbf{\Phi}}\left(\theta_{1}^{a}, \theta_{2}^{a}, \mathbf{y}\right)\right)\right)^{T} \partial_{\theta_{j}} \mathbf{\Phi}\left(\theta_{1}^{a}, \theta_{2}^{a}, \mathbf{y}\right)+\operatorname{cof}\left(\mathcal{J}_{\mathbf{\Phi}}\left(\theta_{1}^{a}, \theta_{2}^{a}, \mathbf{y}\right)\right)^{T} \partial_{t}\left(\partial_{\theta_{j}} \mathbf{\Phi}\left(\theta_{1}^{a}, \theta_{2}^{a}, \mathbf{y}\right)\right)\right), \\
& \mathbf{D}_{4}=\sum_{j} \dot{\theta}_{j}^{b}\left(\operatorname{cof}\left(\partial_{t}\left(\mathcal{J}_{\boldsymbol{\Phi}}\left(\theta_{1}^{a}, \theta_{2}^{a}, \mathbf{y}\right)\right)\right)^{T} \partial_{\theta_{j}} \mathbf{\Phi}\left(\theta_{1}^{a}, \theta_{2}^{a}, \mathbf{y}\right)+\operatorname{cof}\left(\mathcal{J}_{\boldsymbol{\Phi}}\left(\theta_{1}^{a}, \theta_{2}^{a}, \mathbf{y}\right)\right)^{T} \partial_{t}\left(\partial_{\theta_{j}} \mathbf{\Phi}\left(\theta_{1}^{a}, \theta_{2}^{a}, \mathbf{y}\right)\right)\right. \\
& \left.\quad-\operatorname{cof}\left(\partial_{t}\left(\mathcal{J}_{\mathbf{\Phi}}\left(\theta_{1}^{b}, \theta_{2}^{b}, \mathbf{y}\right)\right)\right)^{T} \partial_{\theta_{j}} \mathbf{\Phi}\left(\theta_{1}^{b}, \theta_{2}^{b}, \mathbf{y}\right)+\operatorname{cof}\left(\mathcal{J}_{\mathbf{\Phi}}\left(\theta_{1}^{b}, \theta_{2}^{b}, \mathbf{y}\right)\right)^{T} \partial_{t}\left(\partial_{\theta_{j}} \mathbf{\Phi}\left(\theta_{1}^{b}, \theta_{2}^{b}, \mathbf{y}\right)\right)\right) .
\end{aligned}
$$

We use

- (B.18) and (B.9) for $\mathbf{D}_{1}$,

- (B.2), (B.18) and (B.9) for $\mathbf{D}_{2}$,

- (B.36), (B.25), (B.9), (B.18) and (B.15) for $\mathbf{D}_{3}$,

- (B.36), (B.12), (B.9), (B.2), (B.15), (B.18) and (B.25) for $\mathbf{D}_{4}$,

this gives us the estimate on $\frac{\partial}{\partial t}\left(\mathbf{G}\left(\theta_{1}^{a}, \theta_{2}^{a}, \dot{\theta}_{1}^{a}, \dot{\theta}_{2}^{a}\right)-\mathbf{G}\left(\theta_{1}^{b}, \theta_{2}^{b}, \dot{\theta}_{1}^{b}, \dot{\theta}_{2}^{b}\right)\right)$. Similar arguments give the estimate on $\mathbf{G}\left(\theta_{1}^{a}, \theta_{2}^{a}, \dot{\theta}_{1}^{a}, \dot{\theta}_{2}^{a}\right)-\mathbf{G}\left(\theta_{1}^{b}, \theta_{2}^{b}, \dot{\theta}_{1}^{b}, \dot{\theta}_{2}^{b}\right)$.

- Estimate (3.9): we use the following decomposition

$$
\left(\mathbf{S}\left(\theta_{1}^{a}, \theta_{2}^{a}, \tilde{\mathbf{u}}^{a}, \tilde{p}^{a}\right)-\mathbf{S}\left(\theta_{1}^{b}, \theta_{2}^{b}, \tilde{\mathbf{u}}^{b}, \tilde{p}^{b}\right)\right)_{i}=E_{1, i}+E_{2, i}+E_{3, i}+E_{4, i}+E_{5, i}+E_{6, i},
$$


where

$$
\begin{aligned}
& E_{1, i}=-\left(\left(\mathcal{M}_{\theta_{1}^{a}, \theta_{2}^{a}}-\mathcal{M}_{\theta_{1}^{b}, \theta_{2}^{b}}\right)\left(\begin{array}{c}
\ddot{\theta}_{1}^{a} \\
\ddot{\theta}_{2}^{a}
\end{array}\right)-\left(\mathcal{M}_{\theta_{1}^{b}, \theta_{2}^{b}}-\mathcal{M}_{0,0}\right)\left(\begin{array}{c}
\ddot{\theta}_{1}^{a}-\ddot{\theta}_{1}^{b} \\
\ddot{\theta}_{2}^{a}-\ddot{\theta}_{2}^{b}
\end{array}\right)\right)_{i}, \\
& E_{2, i}=\left(\mathbf{M}_{\mathbf{I}}\left(\theta_{1}^{a}, \theta_{2}^{a}, \dot{\theta}_{1}^{a}, \dot{\theta}_{2}^{a}\right)-\mathbf{M}_{\mathbf{I}}\left(\theta_{1}^{b}, \theta_{2}^{b}, \dot{\theta}_{1}^{b}, \dot{\theta}_{2}^{b}\right)\right)_{i}, \\
& E_{3, i}=\int_{\partial S_{0}}\left(\left|\mathcal{J}_{\mathbf{\Phi}}^{a} \mathbf{t}_{0}\right|-\left|\mathcal{J}_{\mathbf{\Phi}}^{b} \mathbf{t}_{0}\right|\right)\left(\tilde{p}^{a} I-\nu\left(\mathcal{G}\left(\theta_{1}^{a}, \theta_{2}^{a}, \tilde{\mathbf{u}}^{a}\right)+\mathcal{G}\left(\theta_{1}^{a}, \theta_{2}^{a}, \tilde{\mathbf{u}}^{a}\right)^{T}\right)\right) \mathbf{n}_{\theta_{1}^{a}, \theta_{2}^{a}}\left(\Phi^{a}\right) \cdot \partial_{\theta_{i}} \Phi^{a} \\
& E_{4, i}=\int_{\partial S_{0}}\left|\mathcal{J}_{\mathbf{\Phi}}^{b} \mathbf{t}_{0}\right|\left(\tilde{p}^{a} I-\nu\left(\mathcal{G}\left(\theta_{1}^{a}, \theta_{2}^{a}, \tilde{\mathbf{u}}^{a}\right)+\mathcal{G}\left(\theta_{1}^{a}, \theta_{2}^{a}, \tilde{\mathbf{u}}^{a}\right)^{T}\right)\right) \mathbf{n}_{\theta_{1}^{a}, \theta_{2}^{a}}\left(\Phi^{a}\right) \cdot\left(\partial_{\theta_{i}} \Phi^{a}-\partial_{\theta_{i}} \Phi^{b}\right), \\
& E_{5, i}=\int_{\partial S_{0}}\left|\mathcal{J}_{\mathbf{\Phi}}^{b} \mathbf{t}_{0}\right|\left(\tilde{p}^{a} I-\nu\left(\mathcal{G}\left(\theta_{1}^{a}, \theta_{2}^{a}, \tilde{\mathbf{u}}^{a}\right)+\mathcal{G}\left(\theta_{1}^{a}, \theta_{2}^{a}, \tilde{\mathbf{u}}^{a}\right)^{T}\right)\right)\left(\mathbf{n}_{\theta_{1}^{a}, \theta_{2}^{a}}\left(\Phi^{a}\right)-\mathbf{n}_{\theta_{1}^{b}, \theta_{2}^{b}}\left(\Phi^{b}\right)\right) \cdot \partial_{\theta_{i}} \Phi^{b}, \\
& E_{6, i}=\int_{\partial S_{0}}\left|\mathcal{J}_{\Phi}^{b} \mathbf{t}_{0}\right|\left(\left(\tilde{p}^{a}-\tilde{p}^{b}\right) I-\nu\left(\mathcal{G}\left(\theta_{1}^{a}, \theta_{2}^{a}, \tilde{\mathbf{u}}^{a}\right)+\mathcal{G}\left(\theta_{1}^{a}, \theta_{2}^{a}, \tilde{\mathbf{u}}^{a}\right)^{T}-\mathcal{G}\left(\theta_{1}^{b}, \theta_{2}^{b}, \tilde{\mathbf{u}}^{b}\right)-\mathcal{G}\left(\theta_{1}^{b}, \theta_{2}^{b}, \tilde{\mathbf{u}}^{b}\right)^{T}\right)\right) \mathbf{n}_{\theta_{1}^{b}, \theta_{2}^{b}} \cdot \partial_{\theta_{i}} \Phi^{b} \\
& -\int_{\partial S_{0}}\left(\left(\tilde{p}^{a}-\tilde{p}^{b}\right) I-\nu\left(\nabla \tilde{\mathbf{u}}^{a}-\nabla \tilde{\mathbf{u}}^{b}+\left(\nabla \tilde{\mathbf{u}}^{a}-\nabla \tilde{\mathbf{u}}^{b}\right)^{T}\right)\right) \mathbf{n}_{0} \cdot \partial_{\theta_{i}} \mathbf{\Phi}\left(0,0, \gamma_{y}\right) .
\end{aligned}
$$

Then we use

- (B.9) for $E_{1, i}$,

- (B.9), (B.10) and (B.36) for $E_{2, i}$,

- (B.11), (B.28), (B.23) and (B.9) for $E_{3, i}$,

- (B.24), (B.28), (B.23) and (B.9) for $E_{4, i}$,

- (B.24), (B.28), (B.7) and (B.9) for $E_{5, i}$,

- (B.24), (B.17), (B.23) and (B.9) for $E_{6, i}$.

- Estimate (3.10): we use the Lipschitz regularity of $\mathbf{f}_{\mathscr{F}}$ and estimate (B.1),

$$
\begin{aligned}
\| \mathbf{f}_{\mathscr{F}}\left(t, \mathbf{\Phi}\left(\theta_{1}^{a}, \theta_{2}^{a}, \mathbf{y}\right)\right) & -\mathbf{f}_{\mathscr{F}}\left(t, \mathbf{\Phi}\left(\theta_{1}^{b}, \theta_{2}^{b}, \mathbf{y}\right)\right) \|_{L^{2}\left(0, T ; \mathbf{L}^{2}\left(\mathscr{F}_{0}\right)\right)} \\
& \leq C\left\|\mathbf{f}_{\mathscr{F}}\right\|_{L^{2}\left(0, T ; \mathbf{W}^{1, \infty}(\Omega)\right)}\left\|\mathbf{\Phi}\left(\theta_{1}^{a}, \theta_{2}^{a}, \mathbf{y}\right)-\mathbf{\Phi}\left(\theta_{1}^{a}, \theta_{2}^{a}, \mathbf{y}\right)\right\|_{L^{\infty}\left(0, T ; \mathbf{L}^{\infty}\left(\mathscr{F}_{0}\right)\right)} \\
& \leq C T\left\|\theta^{a}-\theta^{b}\right\|_{\Theta_{T}}
\end{aligned}
$$

\section{References}

[1] R. A. Adams. Sobolev spaces. Academic Press [A subsidiary of Harcourt Brace Jovanovich, Publishers], New York-London, 1975. Pure and Applied Mathematics, Vol. 65.

[2] L. Baffico, C. Grandmont, and B. Maury. Multiscale modeling of the respiratory tract. Math. Models Methods Appl. Sci., 20(1):59-93, 2010.

[3] Y. Bazilevs, K. Takizawa, and T. E. Tezduyar. Computational Fluid-Structure Interaction, Methods and Applications. Wiley, 2013.

[4] H. Beirão da Veiga. On the existence of strong solutions to a coupled fluid-structure evolution problem. $J$. Math. Fluid Mech., 6(1):21-52, 2004.

[5] A. Bensoussan, G. Da Prato, M. C. Delfour, and S. K. Mitter. Representation and control of infinite dimensional systems. Systems \& Control: Foundations \& Applications. Birkhäuser Boston, Inc., Boston, MA, second edition, 2007.

[6] M. Boulakia. Existence of weak solutions for the three-dimensional motion of an elastic structure in an incompressible fluid. J. Math. Fluid Mech., 9(2):262-294, 2007.

[7] M. Boulakia and S. Guerrero. A regularity result for a solid-fluid system associated to the compressible Navier-Stokes equations. Ann. Inst. H. Poincaré Anal. Non Linéaire, 26(3):777-813, 2009.

[8] M. Boulakia and S. Guerrero. Regular solutions of a problem coupling a compressible fluid and an elastic structure. J. Math. Pures Appl. (9), 94(4):341-365, 2010. 
[9] M. Boulakia and S. Guerrero. On the interaction problem between a compressible fluid and a Saint-Venant Kirchhoff elastic structure. Adv. Differential Equations, 22(1-2):1-48, 2017.

[10] M. Boulakia, E. L. Schwindt, and T. Takahashi. Existence of strong solutions for the motion of an elastic structure in an incompressible viscous fluid. Interfaces Free Bound., 14(3):273-306, 2012.

[11] L. Bălilescu, J. San Martín, and T. Takahashi. Fluid-rigid structure interaction system with Coulomb's law. SIAM J. Math. Anal., 49(6):4625-4657, 2017.

[12] A. Chambolle, B. Desjardins, M. J. Esteban, and C. Grandmont. Existence of weak solutions for the unsteady interaction of a viscous fluid with an elastic plate. J. Math. Fluid Mech., 7(3):368-404, 2005.

[13] G. Duvaut and J.-L. Lions. Les inéquations en mécanique et en physique. Dunod, Paris, 1972. Travaux et Recherches Mathématiques, No. 21.

[14] G. P. Galdi. An introduction to the mathematical theory of the Navier-Stokes equations. Springer Monographs in Mathematics. Springer, New York, second edition, 2011.

[15] C. Grandmont and Y. Maday. Existence for an unsteady fluid-structure interaction problem. M2AN Math. Model. Numer. Anal., 34(3):609-636, 2000.

[16] P. Grisvard. Elliptic problems in nonsmooth domains, volume 69 of Classics in Applied Mathematics. Society for Industrial and Applied Mathematics (SIAM), Philadelphia, PA, 2011.

[17] M. Hillairet. Lack of collision between solid bodies in a 2D incompressible viscous flow. Comm. Partial Differential Equations, 32(7-9):1345-1371, 2007.

[18] J.G. Houot, J. San Martin, and M. Tucsnak. Existence of solutions for the equations modeling the motion of rigid bodies in an ideal fluid. J. Funct. Anal., 259(11):2856-2885, 2010.

[19] J. Lequeurre. Existence of strong solutions to a fluid-structure system. SIAM J. Math. Anal., 43(1):389-410, 2011.

[20] J.-L. Lions and E. Magenes. Problèmes aux limites non homogènes et applications. Vol. 1. Travaux et Recherches Mathématiques, No. 17. Dunod, Paris, 1968.

[21] Y. Liu, T. Takahashi, and M. Tucsnak. Single input controllability of a simplified fluid-structure interaction model. ESAIM Control Optim. Calc. Var., 19(1):20-42, 2013.

[22] D. Maity, T. Takahashi, and M. Tucsnak. Analysis of a system modelling the motion of a piston in a viscous gas. J. Math. Fluid Mech., 19(3):551-579, 2017.

[23] V. Maz'ya and J. Rossmann. Elliptic equations in polyhedral domains, volume 162 of Mathematical Surveys and Monographs. American Mathematical Society, Providence, RI, 2010.

[24] Š. Nečasová, T. Takahashi, and M. Tucsnak. Weak solutions for the motion of a self-propelled deformable structure in a viscous incompressible fluid. Acta Appl. Math., 116(3):329-352, 2011.

[25] P. A. Nguyen and J.-P. Raymond. Boundary stabilization of the Navier-Stokes equations in the case of mixed boundary conditions. SIAM J. Control Optim., 53(5):3006-3039, 2015.

[26] A. Pazy. Semigroups of linear operators and applications to partial differential equations, volume 44 of Applied Mathematical Sciences. Springer-Verlag, New York, 1983.

[27] J.-P. Raymond. Stokes and Navier-Stokes equations with nonhomogeneous boundary conditions. Ann. Inst. H. Poincaré Anal. Non Linéaire, 24(6):921-951, 2007.

[28] J.-P. Raymond and M. Vanninathan. A fluid-structure model coupling the Navier-Stokes equations and the Lamé system. J. Math. Pures Appl. (9), 102(3):546-596, 2014.

[29] J. San Martín, J.-F. Scheid, T. Takahashi, and M. Tucsnak. An initial and boundary value problem modeling of fish-like swimming. Arch. Ration. Mech. Anal., 188(3):429-455, 2008.

[30] J.A. San Martín, V. Starovoitov, and M. Tucsnak. Global weak solutions for the two-dimensional motion of several rigid bodies in an incompressible viscous fluid. Arch. Ration. Mech. Anal., 161(2):113-147, 2002.

[31] H. Sohr. The Navier-Stokes equations. Birkhäuser Advanced Texts: Basler Lehrbücher. [Birkhäuser Advanced Texts: Basel Textbooks]. Birkhäuser Verlag, Basel, 2001. 
[32] T. Takahashi. Analysis of strong solutions for the equations modeling the motion of a rigid-fluid system in a bounded domain. Adv. Differential Equations, 8(12):1499-1532, 2003.

[33] T. Takahashi, M. Tucsnak, and G. Weiss. Stabilization of a fluid-rigid body system. J. Differential Equations, 259(11):6459-6493, 2015. 